\title{
Structure and Magnetic Properties of Aerosol Nanoparticles of Fe and Its Alloys
}

\author{
Yu. I. Petrov and E. A. Shafranovsky \\ Semenov Institute of Chemical Physics, Russian Academy of Sciences, Kosyguin straß 4, GSP-1, Moscow 119991, Russia \\ Correspondence should be addressed to Yu. I. Petrov, shafr@chph.ras.ru
}

Received 2 March 2012; Revised 5 April 2012; Accepted 12 April 2012

Academic Editor: Zbigniew Tomkowicz

Copyright (C) 2012 Yu. I. Petrov and E. A. Shafranovsky. This is an open access article distributed under the Creative Commons Attribution License, which permits unrestricted use, distribution, and reproduction in any medium, provided the original work is properly cited.

\begin{abstract}
Structure and magnetic properties of aerosol nanoparticles of Fe and its alloys (FeMn, FeNi, FeNiMn, FePt, FeCr, FeCo, and FeCu) have been reviewed. It has been shown that, compared to a bulk material, the particles have a number of specific features being of much fundamental and applied interest. The effect of both a quenched high-temperature Fe modification and its oxides on the structure and magnetism of nanoparticles has been considered in detail. Particular attention has been paid to the recently observed fine structure in the hyperfine field distribution at iron nuclei in Mössbauer spectra for pure iron and its alloys both as a bulk and aerosol nanoparticles. This phenomenon makes it possible to reveal very weak magnetic interactions in the system under study. The plausible origin of these magnetic interactions has been also discussed.
\end{abstract}

\section{Introduction}

Nowadays investigation of the properties of Fe-based nanoparticles is of much interest from fundamental and applied standpoints [1-3]. These particles have high coercivity, and, according to estimates, they are single domain. Short chains of Fe particles and elongated $\mathrm{Fe}_{3} \mathrm{O}_{4}$ particles have long been used for magnetic recording. This is due to their high magnetic anisotropy, large magnetic moment per volume unit, and rectangular hysteresis loop. A rapidly developing nanotechnology [4] offers new applications of magnetic particles. This is, first, development of ultrahighdensity magnetic storage devices based on the giant magnetoresistance effect. Nanoparticles of metastable ball-milled $\mathrm{Cu}$-rich $\mathrm{FeCu}$ alloys approach for this purpose. They show a giant magnetoresistance effect, which can be also used to develop sensors [5-8]. Second, magnetic nanoparticles can be used for drug delivery. Magnetic fine particles can act as a contrast agent for magnetic resonance imaging and can be used for cell separation and various cancer treatments. The latter may be performed, for example, by directing $\mathrm{Fe}_{3} \mathrm{O}_{4}$ particles to tumour sites, using a liquid suspension comprising ultrafine particles surrounded by chains of a blood-plasma substitute. Then one can induce hyperthermia in the targeted cells by a localized heating of magnetite with an RF generator. Magnetic fine particles have also been used to orient biological assemblies and to isolate red blood cells. A number of reviews on magnetic nanoparticles for applying in technology and biomedicine is growing rapidly day by day. The properties of ferromagnetic and antiferromagnetic nanoparticles applied in magnetic fluids for sealing, damping, sensing, and bearing applications have been considered in [9]. The maghemite $\left(\gamma-\mathrm{Fe}_{2} \mathrm{O}_{3}\right)$ and barium ferrite particles are used in hard and floppy computer disks, credit cards, and audio and video recording tapes. Magnetic fluids (ferrofluids) are generally based on either Fe or the ferromagnetic oxide $\left(\mathrm{Fe}_{3} \mathrm{O}_{4}\right)$ suspended in either organic or inorganic liquid (kerosine, water, oil, esters, hydrocarbons). The ferrofluid particles are aligned into rigid chains in an external magnetic field. If these chains are directed along the rotating shaft, they improve the lubricative and sealing properties of liquid lubricant that is essential in designing rotational shafts transmitting motion in vacuum. In practice, this shaft holds the vacuum as high as $10^{-6} \mathrm{~Pa}$ with surface speeds at fluid-shaft interface up to $10 \mathrm{~m} \times$ $\mathrm{s}^{-1}$. If one guides the chains of particles in a magnetic fluid 
between two disks with a magnetic field perpendicular to the disc surface, then their rigid linkage arises. This effect allows one to create new types of brakes and clutches, for example, in automobile industry. Ferrofluids are used for filtering and separation in the mineral processing industry as well as in the Bitter method of magnetic imaging.

Some basic concepts regarding the interactions of magnetic nanoparticles with a static or time-varying external magnetic field have been reviewed in [10]. There were considered current biomedical applications of magnetic nanoparticles including the following topics: (i) magnetic separation of labelled cells and other biological entities; (ii) therapeutic drug, gene, and radionuclide delivery; (iii) radio frequency methods for the catabolism of tumours via hyperthermia; and (iv) contrast enhanced agents for magnetic resonance imaging. Advances in the biomedical applications of magnetic nanoparticles have been presented in [11]. Special attention has been paid to (i) magnetic actuation for in vitro nonviral transfection and tissue engineering, in vivo drug delivery, and gene therapy; (ii) recent clinical results for magnetic hyperthermia treatments of brain and prostate cancer via direct injection, and continuing efforts to develop new agents suitable for targeted hyperthermia following intravenous injection; (iii) developments in medical sensing technologies involving a new generation of magnetic resonance imaging contrast agents, and the invention of magnetic particle imaging as a new modality.

Metastable ball-milled $\mathrm{Fe}_{44} \mathrm{Cu}_{56}$ alloy having a face centered cubic (fcc) structure was discovered to show the Invar effect when a sharp change of thermal expansion coefficient happened nearby the temperature $350^{\circ} \mathrm{C}$ [12]. Therefore this alloy may be applied as a temperature switch. A number of suggestions how to improve and cheapen the elements of microelectronics using aerosol nanoparticles of iron alloys have been given in [13].

From a scientific point of view it is important to follow how changing the properties of solid matter while transiting to nanoparticles as well as the role played by $\mathrm{Fe}$ oxides and high-temperature $\gamma$-Fe modification in Fe-based alloys nanoparticles. This paper summarizes the studies on structure and magnetic properties of aerosol nanoparticles of iron and its alloys mainly obtained at Semenov Institute of Chemical Physics RAS.

\section{Sample Preparation}

Initial Fe-based alloys were produced by alloying components with a purity of $99.9 \%$, a total mass of which was about $1.5 \mathrm{~g}$, in a $10 \mathrm{~kW}$ RF generator operated at a frequency of $440 \mathrm{kHz}$. In order to avoid contamination from the crucible, an inductor was specially designed which provided the alloy components to levitate in an Ar atmosphere. Eddy currents ensured a homogeneous mixing of alloy components within a melted drop. After exposing the drop at about 2000$2500^{\circ} \mathrm{C}$ for a few minutes, the generator was turned off, and the drop fell down in a massive copper vessel. The alloy composition was monitored by specimen weighing before

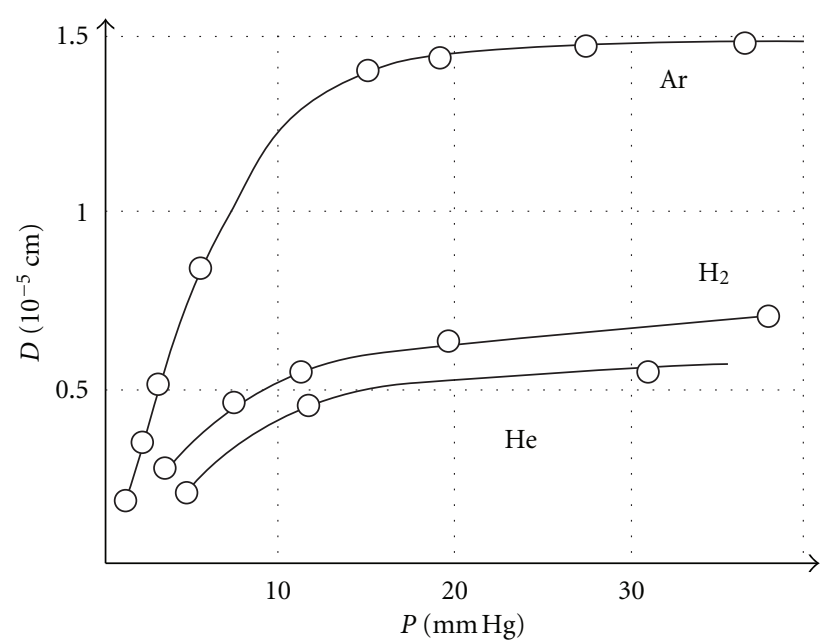

Figure 1: Dependence of aerosol Al particles size on pressure and gas species [16].

and after alloying components and by X-ray measurements. Weight losses did not exceed 1 to $2 \%$.

Aerosol particles were produced by fast evaporation of the iron and initial alloys, predegassed in vacuum, in an Ar atmosphere typically at $400 \mathrm{~Pa}$ pressure from a tungsten wire or Ta capsules heated by ac power (gas evaporation technique). It is very essential that the particles of alloys (e.g., AgCu [14]) or compounds (e.g., CdSe [15]) keep the stoichiometric composition of the initial substance. It has been first established for aluminium that near spherical aerosol particles are formed when evaporating metal in an inert gas atmosphere, and the average size of which can be easily varied by changing the pressure and the gas species (Figure 1) [16]. The features of this technique were discussed in detail elsewhere [17-19].

It was assumed that the nucleation and growth of particles occur in two stages within the condensation zone (Figure 2, [18]). Small atomic aggregates arise in the immediate vicinity of evaporator (inner border of "condensation zone" (3)). Diffusing through the gas, these small aggregates coalesce via collisions to form the larger particles. The process completes at outside border of the "condensation zone" (4) where the gas temperature reduces as much as the cooled particles cannot already coagulate. As the gas pressure increases, the condensation region stretches and appears inside the vacuum chamber (2). At the gas pressure higher than several thousands of $\mathrm{Pa}$ a well visible cloud of the aerosol particles is formed around the evaporator. The cloud moves through the vessel by gas convection flows (7). When the vessel walls appear inside the "condensation zone," this takes place at low $\left(<10^{-2} \mathrm{~Pa}\right)$ gas pressures or in the case of a small vessel volume, then the average size of particles monotonically increases as a displacement of the walls from inner to outer border of the "condensation zone" occurs. Processes of nucleation and growth of particles can be initiated or continued at the vessel walls as well as on a surface of any object put in if the temperature of its surface favors. The appearing aerosol particles have nearly a 


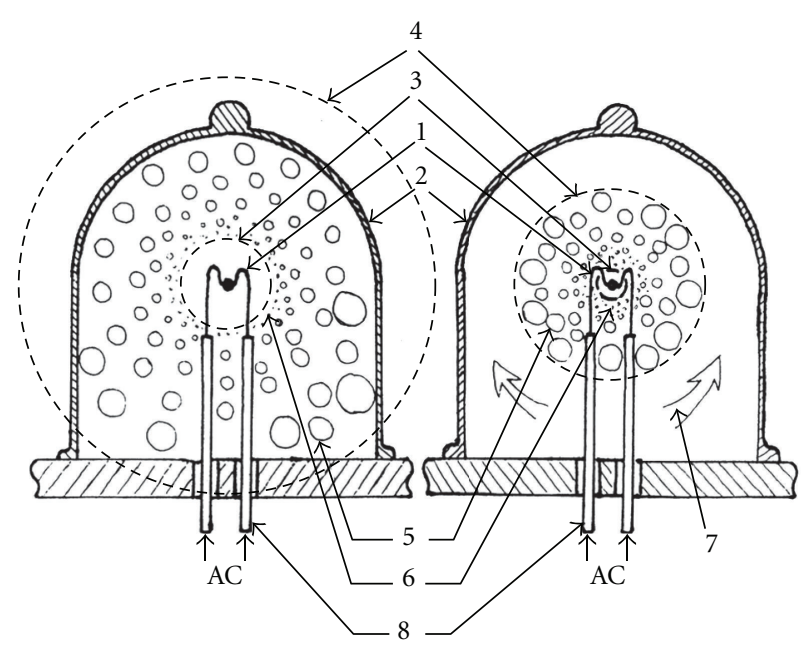

(a)

(b)

FIGURE 2: Inert gas pressure-dependent arrangement of condensation zone border. (a) Low inert gas pressure. An imaginary outer boundary of the condensation zone is beyond the vacuum chamber. A deposited particle size grows with increasing the inert gas pressure. (b) High inert gas pressure. The outer boundary of the condensation zone is within the vacuum chamber. A deposited particle size does not practically change with increasing the inert gas pressure. (1) Evaporator; (2) vacuum chamber; (3) inner boundary of the condensation zone; (4) imaginary (left) or real (right) outer boundary of the condensation zone; (5) aerosol particles; (6) clusters; (7) gas convection flows; (8) electrodes.

spherical shape and in all cases a particle size distribution appears rather narrow $[18,19]$. In our experiments the aerosol particles were precipitated on the walls of a glass beaker or metallic cylinder of $10 \mathrm{~cm}$ in diameter which was located inside a large vacuum chamber of $0.12 \mathrm{~m}^{3}$ volume. An average size of the metallic core of particles prepared in these conditions at Ar pressure $400 \mathrm{~Pa}$ was equal to $13-15 \mathrm{~nm}$ whereas diminishing Ar pressure to tens of Pascals allows one to produce particles of smaller size.

Ultrafine particles of metals and alloys can easily selfignite at the first contact with air. However, if they are kept some time in the inert gas, they are covered with a passivating oxide shell, preventing the further particle oxidation. To completely avoid a self-ignition of the deposits upon exposure to air, the aerosol particles were kept in a preparation chamber with the volume of $0.12 \mathrm{~m}^{3}$ for $12 \mathrm{~h}$ in $\mathrm{Ar}$ atmosphere at the same pressure. After this procedure the particles were covered with a thin passivating oxide shell allowing further handling of the powder in ambient atmosphere [20]. For example, iron particles in diameter of about $50 \mathrm{~nm}$ were aged in air for 20 years without any noticeable change of the oxide content. As a rule, the primary oxide on particles surface is amorphous, and its content can reach $40 \%-50 \%$ for particles of size smaller than $10 \mathrm{~nm}$. It crystallizes during heating powders up to several hundreds of Celsius degree in a high vacuum. Note that small particles of ferromagnetic metals and alloys form long chains after their moving-away out of the aggregation region due to the arising forces of magnetic attraction with their cooling below Curie point. A unique peculiarity of the gas condensation method is in an ability to freeze high-temperature states in aerosol particles during their rapid quenching with a rate of $10^{5}-10^{6}$ degree per second in the course of their preparation.

In recent years many attempts were undertaken to update the gas evaporation technique by applying various evaporation methods in the inert gas atmosphere (e.g., laser, radio frequency, arc, plasma ones, etc.). However, their low yield remains the main disadvantage of this technique. For example, with a thermal evaporation of metal the yield of high-dispersive powders reaches several hundreds of milligrams a day. The arc evaporation gives the higher yield, but a spraying of large drops takes place which cannot be excluded.

A significant progress has been reached owing to a levitation-flow aerosol generator developed in Semenov Institute of Chemical Physics by Guen and Miller [21, 22] and Jigach et al. [23] (see also [18]). It permits manufacturing aerosol particles with a size of about 30 to $200 \mathrm{~nm}$ and yielding several grams per hour. A large drop of metal (alloy) is levitated and heated with a radio frequency field of the hardening generator of $6.5 \mathrm{~kW}$ output power inside a quartz tube through which the inert gas laminarly flow in a closed cycle at the atmospheric pressure. The evaporated atoms form aerosol particles within a narrow "condensation zone" close to the drop. These particles are entrained with the gas flow and separated on a fabric filter. The melted drop is continuously supplied with metal by feeding its wire. A powder collected on the filter is periodically put off into a special reservoir. The generator can operate all day and night with a short-time stop for technical service.

\section{Apparatus}

A filterless $\mathrm{Cr}$ characteristic radiation (tube voltage $33 \mathrm{kV}$, current $16 \mathrm{~mA}$ ) was used in early works for X-ray diffraction (XRD) measurements. X-ray photographs of the samples were recorded on a Kodak X-Omat film (exposure 10$15 \mathrm{~min}$ ) arranged asymmetrically in a conventional $57.3 \mathrm{~mm}$ Debye-Scherrer camera. The lattice constants of the samples were determined by extrapolation of the data to the $90^{\circ} \mathrm{Bragg}$ angle with accuracy $\pm(0.001-0.0005) \AA$. Thermal expansion was measured with a specially designed X-ray vacuum chamber of $70 \mathrm{~mm}$ in diameter $[18,24]$. A sample in diameter of about $0.3 \mathrm{~mm}$ being a suspension of aerosol powder in Bakelite was stuck to a central part of rotating Pt thin wire of $0.1 \mathrm{~mm}$ in diameter. The Pt wire was heated by a.c. power up to various temperatures and X-ray patterns were taken. The sample was preheated in vacuum at 150 $200^{\circ} \mathrm{C}$ to carbonize the Bakelite. The chamber allowed one to get $9 \mathrm{X}$-ray patterns of $6 \mathrm{~mm}$ in width for one run. A sample temperature was evaluated by displacement of reverse Pt Xray lines with accuracy of up to $15-20^{\circ} \mathrm{C}$.

In recent works structural characterization of powder samples under study was performed by analyzing XRD patterns recorded with a Siemens D5000 powder diffractometer with $\mathrm{Cu} \mathrm{K} \mathrm{K}_{\alpha}$ incident radiation $(\lambda=1.5406 \AA)$ and a graphite 
monochromator in a $\theta-2 \theta$ Bragg-Brentano geometry. The XRD data were analysed by Rietveld refinements using the MAUD program [25]. In some cases the interplanar spacings of the ordered structures were determined with the help of the GATAN program (http://www.gatan.com/) using the Fourier analysis for the selected parts of the high resolution TEM (transparent electron microscope) patterns of the particles. The lattice constants at all nanoparticles were practically identical to those at the bulk.

Mössbauer spectra were collected at 300, 100, 80, and $4.2 \mathrm{~K}$ using a conventional transmission Mössbauer spectrometer with constant acceleration and a ${ }^{57} \mathrm{Co}(\mathrm{Cr}$ ) (in the recent works ${ }^{57} \mathrm{Co}(\mathrm{Rh})$ ) source. Velocity calibration was done using a $25 \mu \mathrm{m}$ foil of Armco iron. Recording spectra were fulfilled with a 512-channel multichannel analyzer under a saw-toothed motion of the source. Therefore the folding procedure was applied in the course of processing spectra on computer with the LOREN program in the early works and the NORMOS program [26] in the recent ones. Since as-fresh aerosol particles were weakly linked with each other (porous powders), large counting background was present reducing the probability of the Mössbauer effect. However, compacting powders placed between two Ta plates or in agate mortar prior to Mössbauer measurements increased the relative Mössbauer effect by several times and entirely suppressed superparamagnetism of nanoparticles. X-ray patterns of the powders after such treatment did not reveal any changes in their structure as well as in a lattice parameter.

\section{Results}

4.1. Magnetism of Iron Nanoparticles. The correlation between the structure and magnetic properties of the aerosol Fe particles of $\sim(15-17) \mathrm{nm}$ in size together with Fe oxide shell has been investigated by X-ray, TEM, SQUID magnetometer and Mössbauer spectra, and the obtained results are summarized in [18]. Size distribution obtained after treating the TEM patterns of as-fresh Fe particles (sample I) and the same particles after their heating in vacuum $2.7 \times 10^{-3} \mathrm{~Pa}$ at $500^{\circ} \mathrm{C}$ for $1 \mathrm{~h}$ (sample II) is given in Figure 3. Maxima on the curves correspond to a size of $13.4 \mathrm{~nm}$ for sample I and $13.2 \mathrm{~nm}$ for sample II. The particle average weighed sizes $(d)$ and ones weighed with the volume $\left(\left\langle d^{3}\right\rangle\right)^{1 / 3}$ equal 14.9 and $17.0 \mathrm{~nm}$ for sample I and 15.2 and $17.6 \mathrm{~nm}$ for sample II, respectively. From the comparison of the X-ray line broadening for samples I and II the average particle size appeared to equal $11 \pm 2$ and $15 \pm 2 \mathrm{~nm}$, respectively.

Prior to discussing the obtained results concerning magnetic properties of aerosol Fe nanoparticles, it is reasonable to make some theoretical notes on magnetism of an ensemble of small particles. With decreasing size of multidomain particles a single domain state becomes energetically more favorable $[1,2]$. As this takes place, the probability of the Browniankind motion for the saturation magnetization vector of one particle $\mathbf{I}_{S}$ increases. Thermal fluctuations can give rise to rotations of $\mathbf{I}_{S}$ only if they overcome the energy barrier $E_{B}$. Usually, one takes $E_{B}=K V \sin ^{2} \varphi$, where $\varphi$ stands for the angle between the $\mathbf{I}_{S}$ and the easy magnetization of particle (EM), $K$ for anisotropy constant, and $V$ for a particle volume. The probability of thermal fluctuations is proportional to $\exp \left(-E_{B} / k T\right)$. The total magnetization for ensemble of $N$ particles per volume unit, $\mathbf{M}_{S}=N \mathbf{I}_{S}$, arising in the external field $\mathbf{H}_{S}$ which is sufficient to attain the magnetic saturation of the ensemble, after switching off the field, tends to zero due to the Brownian motion of $\mathbf{I}_{S}$ for individual particles, following the law

$$
\mathbf{M}(t)=\mathbf{M}_{S} \exp \left(-\frac{t}{\tau}\right)
$$

where $\tau$ is a relaxation time. Thus, an ensemble of particles behave itself like a swarm of paramagnetic atoms with the large magnetic moment $\mathbf{I}_{S}$. This phenomenon is called superparamagnetism. The theory assumes that the vector $\mathbf{I}_{S}$ is in any one energy minimum for a long time and then quickly transits from one minimum to another. Such a model of discrete orientations of the vector $\mathbf{I}_{S}$ approximates the relaxation time $\tau$ by the expression

$$
\tau=\tau_{0} \exp \left(+\frac{E_{B}}{k T}\right),
$$

where the energy barrier $E_{B}=K V$ in a case of uniaxial anisotropy. In various works the value $\tau_{0}$ is taken between $10^{-9}$ and $10^{-10} \mathrm{~s}$. If the particles are fixed (powder), then transition of $\mathbf{I}_{S}$ from one energy minimum to another takes place very quickly at certain values of $V_{B}$ and $T_{B}$, called a block volume and block temperature. When attaining these values, the vectors $\mathbf{I}_{S}$ of particles are arranged quasi parallel to the external field. By changing the direction of magnetic field to the opposite one, one obtains the hysteresis loop characteristic to ferromagnetism. Particles for which $V<$ $V_{B}$ or $T>T_{B}$ show a paramagnetic behavior. Increase of $V$ or decrease of $T$ transfer them to a ferromagnetic state and vice versa. Due to a particle size distribution, the specimen contains superparamagnetic and ferromagnetic fractions in a different ratio. It is the case that has impact on the shape of field-cooled (FC) and zero-field-cooled (ZFC) curves (Figure 4). On the other hand, if the particles can move freely in space (collodion solutions), then their anisotropy plays no special role, and they behave themselves like a Brownian motion of molecules of a paramagnetic gas, which does not manifest a hysteresis loop.

Provided that directions of $\mathbf{H}$ and EM coincide $(\vartheta=0)$ and $\varphi \ll 1$, the theory gives a so-called anisotropy field of particle $H_{K}=2 \mathrm{KV} / \mathbf{I}_{S}[18,27]$. At the same time, a direction of $\mathbf{I}_{S}$ close to $\mathbf{H}$ can be stable or unstable depending on the fulfillment of inequalities $H \leq-H_{K}$ or $H \geq H_{K}$, respectively. One supposes that after attaining the value of magnetic field $H_{S}= \pm H_{K}$, the vector $\mathbf{I}_{S}$ quickly reverses its direction by coherent rotation of all spins of a particle [27]. The initially disoriented vectors $\mathbf{I}_{S}$ of the ensemble of particles are ordered in the magnetic field $\mathbf{H}$. Saturation magnetization of specimen, $\mathbf{M}_{S}$, is attained when $H_{S}=+H_{K}$, and all the vectors $\mathbf{I}_{S}$ are aligned parallel to $\mathbf{H}$. This state holds also by changing the field to a value $H_{S}=-H_{K}$. Then, owing to instability of positions of the vectors $\mathbf{I}_{S}$ a net 


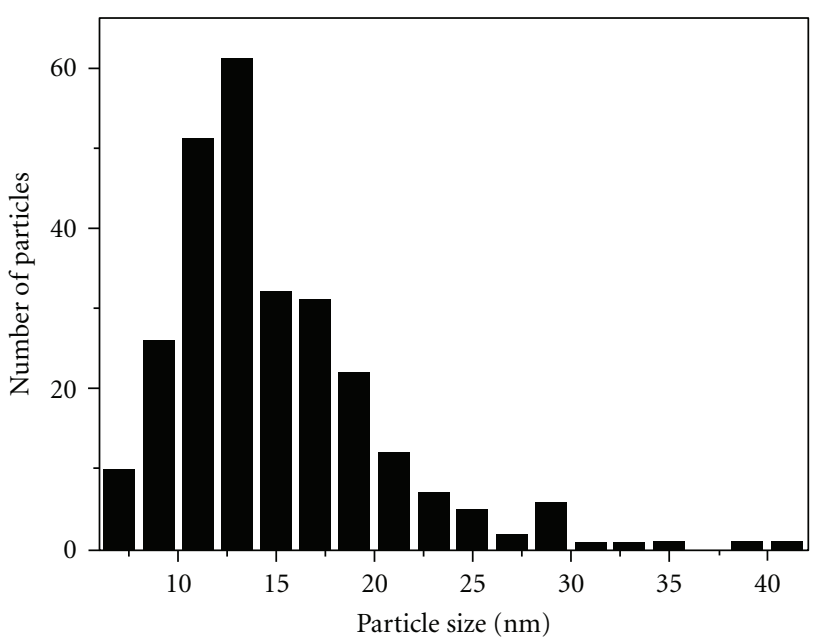

(a)

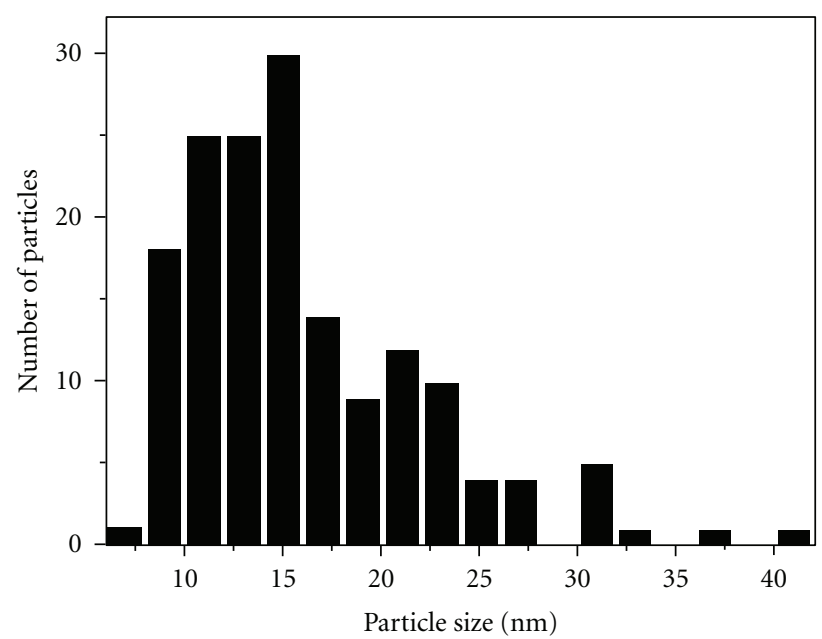

(b)

FIgURE 3: Distribution of the Fe particle size based on treating TEM bright field pattern of the particle ensemble. (a) Sample I; (b) sample II.

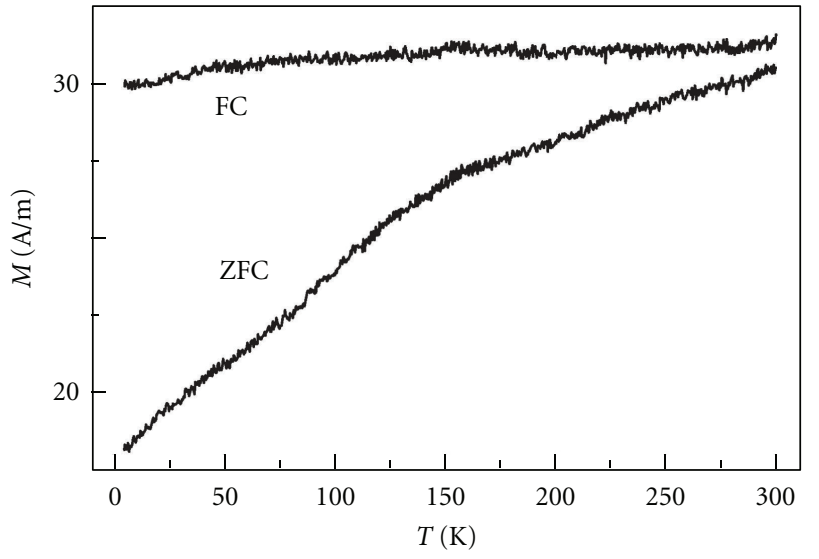

(a)

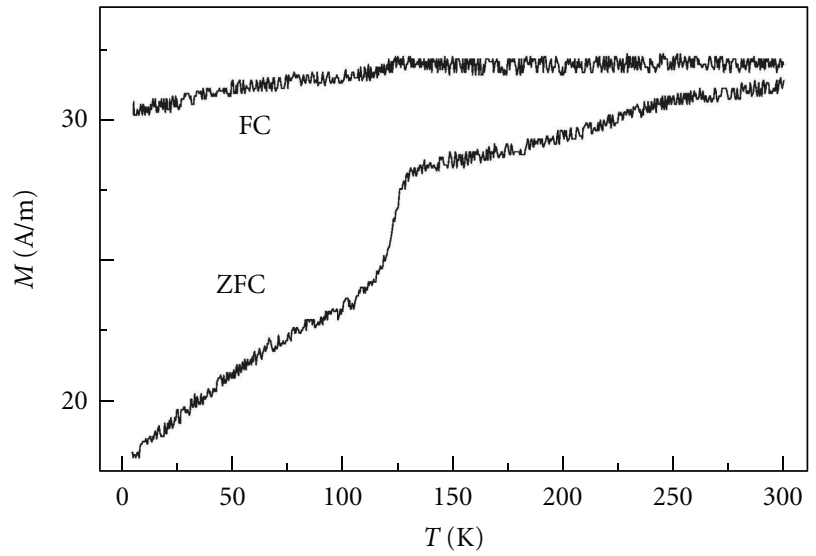

(b)

FIGURE 4: ZFC-FC magnetization curves as a function of temperature for a field of $H=10^{-2} \mathrm{~T}$. (a) Sample I; (b) sample II.

vector $\mathbf{M}_{S}$ sharply turns through $180^{\circ}$. A similar turn of the reverse vector $-\mathbf{M}_{S}$ to the initial state occurs by the following increase of the field to $H_{S}=+H_{K}$. The obtained hysteresis loop appears rectangular with a coercivity $H_{C}=H_{K}$.

In a case when the directions of $\mathbf{H}$ and EM do not coincide $(\vartheta \neq 0)$ calculations become complicated. For some intermediate values of angle $\vartheta$ between 0 and $90^{\circ}$ the hysteresis loops were calculated by Stoner and Wohlfarth [27]. As the angle $\vartheta$ increases, the hysteresis deviates more and more from a rectangular shape, becomes narrower, and bends. However the anisotropy field $H_{K}$ always defines the fusion points (FS) of loop branches accounting for the saturation magnetization of the system. In these points the magnetic state of the ensemble of particles appears unstable because under the action of thermal fluctuations the vectors $\mathbf{I}_{S}$ can easily change their orientation overcoming the energy barriers. Hence, the magnetic fields accounting for the fusion points of hysteresis loops are necessary for the full magnetic reversal of specimens.
In many early works (see in [18]) one has paid mainly attention to examination of coercivity dependence for an ensemble of small particles on their size and package density. Later an emphasis moved to the anisotropy constant dependence on the particle shape and interaction of particles with each other considering their superparamagnetism. However in all the cases the anisotropy constant $K$ was defined using the formula $H_{C}=A K V / \mathbf{I}_{S}$, where coefficient $A$ was varied in different theories. This formula can be valid only for a rectangular hysteresis loop of sample when $A=2$. If one aligns mentally EM axes of all particles along a magnetic field $\mathbf{H}(\vartheta=0)$, then $H_{C}=H_{K}=2 \mathrm{KV} / \mathbf{I}_{S}$. At the same time a real particle system shows an inclined hysteresis loop (Figure 5). Hence, estimates of $K$ using the above formula cannot be considered correct. Besides, $H_{C}$ is very sensitive to the package density of particles and specimen deformation. $H_{C}$ depends theoretically also on mechanism of magnetic reversal for the particle chains changing (for $\vartheta=0$ ) from 0.09 to $0.27 \mathrm{~T}(n=2$, chains consisting of 2 particles $)$ 


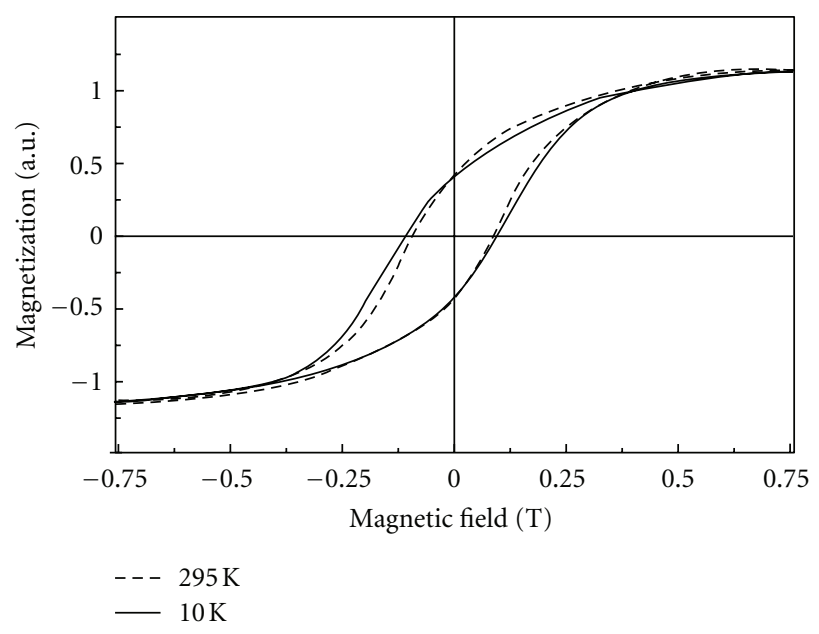

(a)

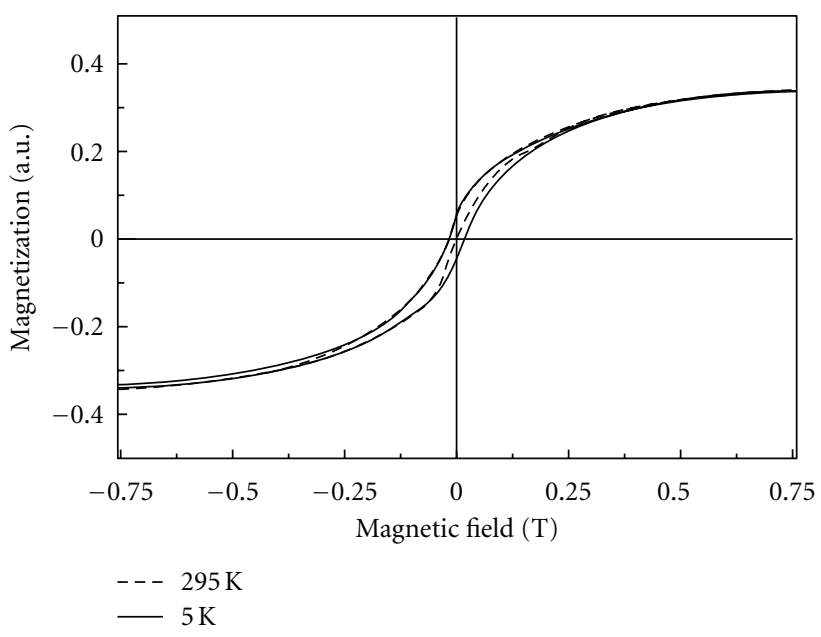

(b)

Figure 5: Hysteresis loop curves for extreme temperatures. (a) Sample I; (b) sample II.

and from 0.27 to $0.64 \mathrm{~T}(n \rightarrow \infty)$ at transiting from the fanning mechanism to the parallel rotation mechanism. For random distributed chains these values $H_{C}(\vartheta=0)$ must be multiplied by factor 0.479 .

Since a real ensemble of nanoparticles shows an inclined hysteresis loop, a value $H_{C}$ characterizes in no way a total irreversible remagnetization of the particles chains. This happens only when a magnetic field attains values $H_{K}=$ $\pm 2 K V / \mathbf{I}_{S}$. To calculate $K$, it is necessary to determine a field $H_{K}$ for the fusion points of loop branches. For sample I and sample II $H_{K} \approx 0.55 \mathrm{~T}$. Using this value of $H_{K}$ and taking a saturation magnetization of unit volume of particles $\mathbf{I}_{S} / V$ equal that for the bulk $\left(1.7 \times 10^{3} \mathrm{~T} \mathrm{~m}^{-3}\right)$, we get $K_{H}=4.7 \times 10^{5} \mathrm{~J} \mathrm{~m}^{-3}$ that is one order higher than the crystalline anisotropy constant for the massive iron $(K=$ $\left.4.2 \times 10^{4} \mathrm{~J} \mathrm{~m}^{-3}\right)$. It is doubtful that individual particles could have such a large anisotropy constant.

An enhanced anisotropy constant for ensemble of small ferromagnetic particles was time and again reported previously too. In a work [28] for $\alpha$-Fe particles with a size of 3-40 nm produced by gas condensation method a uniaxial anisotropy constant $K$ was determined to equal $(2-3) \times 10^{5} \mathrm{~J} \mathrm{~m}^{-3}$. Authors of the work wondered why the anisotropy constant $K$ is so large. Meanwhile, Jacobs and Bean [29] had already explained a strong increase of $K$ compared to the crystalline anisotropy constant by an availability of chains of spherical particles in specimens.

There is another standpoint explaining high values of anisotropy constant and saturation magnetization $M_{S}$ which is based on a peculiar structure of the very nanoparticles and surface effects $[18,30-32]$. It is supposed that large strains arise because of break of bonds on the particle surface, which results in a particle compression and a strong disordering of surface spins. Kodama et al. [30-32] considered that the canting spins freeze themselves into a spin-glass-like phase at temperatures below $50 \mathrm{~K}$. Thus, the surface spins had multiple configurations. They connected the temperature dependence of coercivity and hysteresis loop

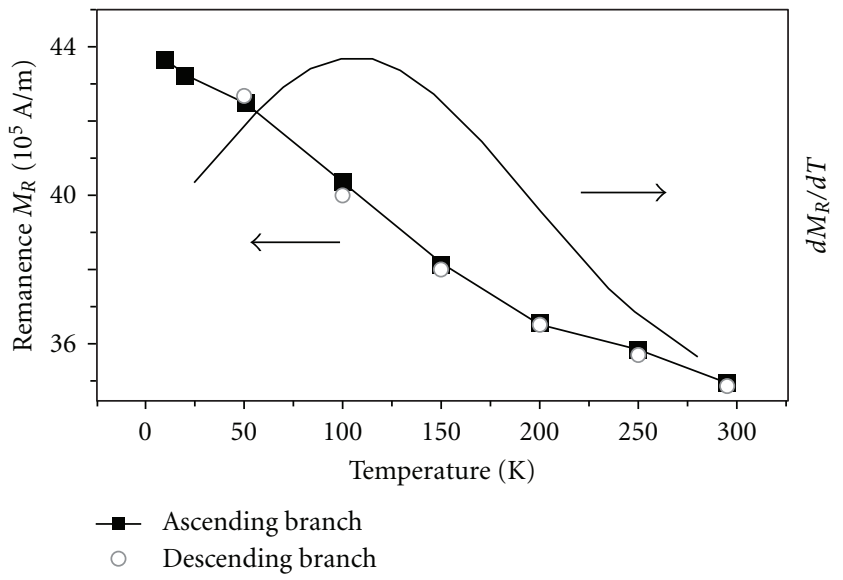

Figure 6: Temperature dependence of $M_{R}$ and its derivative for sample I.

shift for nanoparticles $\mathrm{NiFe}_{2} \mathrm{O}_{4}$ at $T<50 \mathrm{~K}$ with a freezing of disordered surface spins. In their opinion, coupling with the frozen disordered surface spins makes core spin reversal more difficult that results in a high value of $H_{K}$. Due to the exchange coupling between the surface and core spins, field cooling can select a surface spin configuration which favored the particles being magnetized in the field-coding direction, hence resulting in a shifted hysteresis loop below $50 \mathrm{~K}[31,32]$. Surface conditions, in particular a state of oxide shell, play certainly an important role [18]. However, a wide-world point of view on existence of surface tensions compressing particles is erroneous [33-35].

As temperature goes down, smaller and smaller particles in sample I are transiting to a stable ferromagnetic state, therefore the magnetization goes up. One can take this occasion to estimate anisotropy constant. Figure 6 shows a temperature dependence of magnetization for ascending and descending branches of hysteresis loop for sample I. 
Differentiating an averaged curve, we get a distribution of blocking temperatures corresponding to the particle size distribution (Figure 6). A maximum of the derivative at $T_{B}=$ $110 \mathrm{~K}$ must be referred to the mean diameter of particle core $\langle D\rangle=11.6 \mathrm{~nm}$. Now we can use the condition defining a boundary to transit between superparamagnetic and stable ferromagnetic states of particles [18]

$$
\frac{K V}{k T}=25 .
$$

It is quite acceptable because this expression does not include magnetic fields and a remanence is measured at $H=0$. Then we get $K_{S}=25 \mathrm{kT} / V=150 \mathrm{kT} / \pi(\langle D\rangle)^{3} \approx 5.6 \times 10^{4} \mathrm{Jm}^{-3}$. This value of $K_{S}$ is close to the anisotropy constant for massive iron $\left(4.2 \times 10^{4} \mathrm{Jm}^{-3}\right)$. Meanwhile it is worthy of note that the estimate of $K_{S}$ is highly rough due to an approximate definition of $T_{B}$ and arbitrariness of condition (3). It should be noted that the lacking of any peculiarity on the ZFC curve for sample I (Figure 4) at $T=110 \mathrm{~K}$ is likely due to the different conditions for measuring the ZFC curve (an initial disordered state of spins) and the hysteresis loop (an initial arrangement of spins ordered at $M_{S}$ ). A big distinction of the anisotropy constants $K_{\mathrm{H}}$ and $K_{S}$ really stems from their different origin. The constant $K_{H}$ corresponds to a full magnetic reversal of specimen, and the constant $K_{S}$ is related to the particle transition from one state into another.

Hysteresis loop curves for both samples are given in Figure 5. Their shape remains almost the same in the whole temperature range $5-300 \mathrm{~K}$ but differs considerably between the two samples. Sample II has a very narrow hysteresis loop and its shift is slightly expressed at any temperature used whereas the greatly opened hysteresis loop at sample I noticeably shifts with decreasing temperature giving rise to growth of coercivity $H_{C}$ and remanence $M_{R}$ of as-fresh Fe particles (Figure 7).

A shape of ZFC and FC curves (Figure 4) enables to judge the relaxation behavior of samples I and II and to specify a structure of the particles oxide shell. When temperature increases from $4 \mathrm{~K}$, the magnetic moments of nanoparticles, frozen initially in random way, tend to align with the directions of easy axes nearest to the magnetic field applied when measuring magnetization of a sample. As a result, the magnetization of the latter grows. At the same time in the powder with a definite particle size distribution the more and more large fractions simultaneously undergo a ferromagnetic $\rightarrow$ superparamagnetic transition and the magnetization increase gradually slows down. A shape of ZFC and FC curves is dependent in a complicated way on size distribution, character of interparticle interactions as well as on the applied field, measurement rate, and time. Therefore, to get unambiguous conclusions on the magnetic properties of the system studied by analyzing the ZFC and FC curves is not always possible.

4.2. Inheritance of High-Temperature Fe Modification States. As known, Fe-rich alloys show anomalous physical properties, in particular, a weak temperature dependence of thermal expansion coefficient at low temperatures (Invar effect) and its sharp increase close to Curie point (anti-Invar effect). Anomalies of thermal expansion are accompanied with change of magnetic state of alloys. There are many model explanations of Invar effect which are discussed in reviews of Schlosser [36], Window [37], Wassermann [38], and Wassermann and Entel [39]. The most of models are based on supposition of availability in alloys of different kinds of inhomogeneities (structure, concentration, magnetic, etc.). Actually, such models are reduced to various local interactions of iron atoms with their nearest neighborhood. On the other hand, analyzing thermodynamic data as well as the results of electric and magnetic measurements for pure iron, Weiss et al. [40-42] came to the conclusion that its high-temperature fcc-modification could exist in twospin electron states. One of these states, high-spin (HS), is ferromagnetic. It has a lattice constant $a=3.64 \AA$ and a magnetic moment $\mu \approx 2.8 \mu_{B}$ ( $\mu_{B}$ is Bohr magneton). The other, low-spin (LS), is antiferromagnetic with $a=3.54 \AA$ and $\mu \approx 0.5 \mu_{B}$. The actual thermal expansion of the fcc iron and its alloys is defined by the joint action of both states. Weiss [41] suggested a model scheme from two energy levels populated by HS and LS states and separated by the energy gap $\Delta E \approx 0.04 \mathrm{eV}$. For ferromagnetic FeNi, FePt, FePd alloys the ground state is the HS state while in the case of antiferromagnetic fcc Fe, FeMn, FeC materials the ground state appears to be the LS state. A relative population of the energy levels is defined by the Boltzman factor $\exp (-\Delta E / k T)$. A value $\Delta E$ depends on alloy composition, and it can change sign. For example in $\mathrm{FeNi}$ alloy, the inversion of levels takes place at $\mathrm{Ni}$ concentration close to $30 \%$. The relation of HS and LS components with different lattice constants determines a thermal expansion coefficient for iron and its alloys, making it practically independent of temperature in a certain temperature range (Invar effect). Weiss's works $[41,42]$ stimulated a number of important quantum mechanical calculations, elucidating the existence of multispin states in a crystal lattice of transition metals and their alloys (see in [43]). Since some models suppose availability in Fe-rich alloys of microinhomogeneities with a size of some micrometers, it is of great interest to study properties of ultrafine particles of both the pure iron and its alloys.

The striking results gave Mössbauer spectra of "abnormal" Fe particles with an average size of $50 \mathrm{~nm}$ (Figure 8) $[43,44]$. It is seen that these spectra can be decomposed into two components corresponding to hyperfine fields at Fe nuclei $H_{1}=36.6 \mathrm{~T}$ and $H_{2}=33.0 \mathrm{~T}$ (Figure 8(a)). Annealing of particles at $800^{\circ} \mathrm{C}$ in $\mathrm{Ar}$ atmosphere for $30 \mathrm{~min}$ followed by quenching down room temperature resulted in a significant growth of intensity of the component with the high hyperfine field $H_{1}$ (Figure $8(\mathrm{~b})$ ). These results have been obtained only with the powders previously prepared by Guen in a levitation-flow generator at $\mathrm{Ar}$ pressure of about $1 \times 10^{5} \mathrm{~Pa}$ and an extremely rapid quenching rate. The powders were kept in air for several decades. Slight traces of oxide were observed only in the Mössbauer spectra and absent in X-ray patterns. Auger spectra recorded after etching the samples by argon ions did not manifest any impurity elements in the particles. Later, X-ray analysis showed a slight 


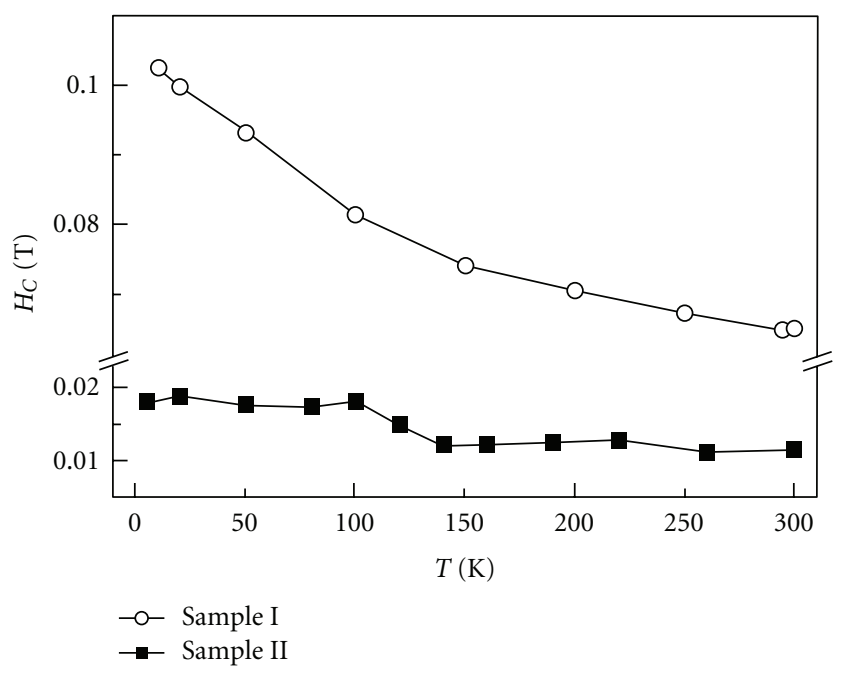

(a)

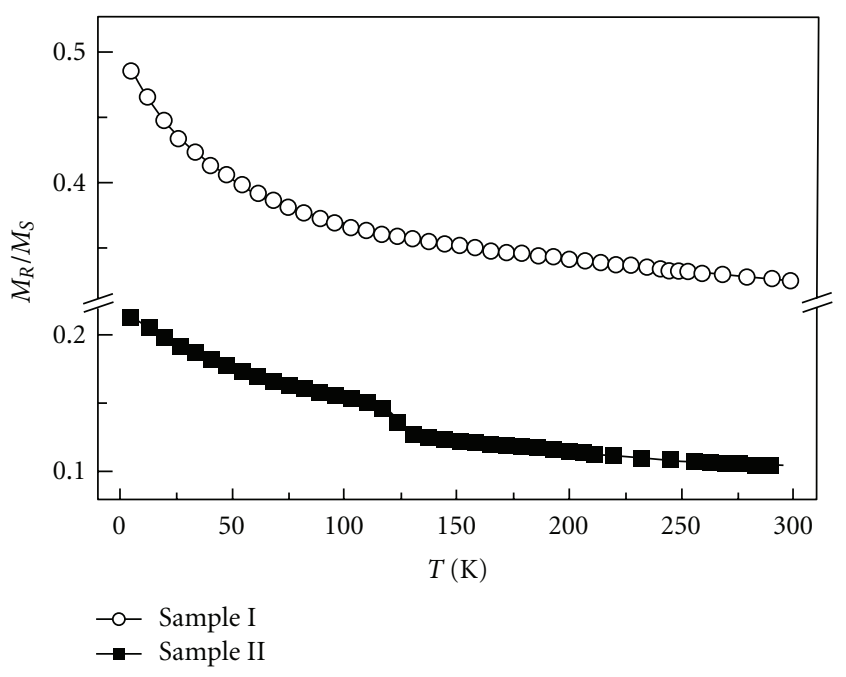

(b)

Figure 7: (a) Temperature dependence of coercivity, $H_{C}$, for samples I and II. (b) Temperature dependence of remanence, $M_{R}$, for samples I and II $\left(M_{S}\right.$ is saturation magnetization).

admixture of cobalt. However, a specially prepared powder of iron doped with cobalt showed the typical sextet with narrow linewidths. Similar pattern was also in spectra of $7 \mathrm{~nm}$ and larger Fe particles produced by the levitationflow generator. In X-ray patterns of initial and annealed samples, any extraneous lines, for example, oxide ones, were not observed except the bcc Fe lines corresponding to the proper lattice constant. Thus, both components of the Mössbauer spectrum (Figure 8) are related to the bcc lattice though, as well known, it displays only one hyperfine field $H_{2}=33 \mathrm{~T}$. Since the hyperfine field $H_{1}=36.6 \mathrm{~T}$ was not found at the bcc Fe particles of $7 \mathrm{~nm}$ in diameter, it will not be able to ascribe this field to action of surface layer for large particles at which, in addition, a fraction of this layer is negligible. Therefore we can make the conclusion that the high hyperfine field represents inheritance of the HS state of the high-temperature fcc modification in the bcc iron lattice stable at $20^{\circ} \mathrm{C}$. As a consequence, after annealing the sample the lattice parameter of bcc Fe is slightly larger $(2.873 \pm 0.004) \AA$ compared to its normal value $(2.866 \pm 0.002) \AA[44]$. Note that the Mössbauer spectra in Figure 8 contain additionally a small central paramagnetic peak which can be ascribed to the fcc lattice itself though Xray patterns do not show it. However the study of thermal expansion of $30 \mathrm{~nm}$ iron particles revealed a presence of slight amount of the fcc modification at $20^{\circ} \mathrm{C}$ after their rather slow cooling from temperature higher than $800^{\circ} \mathrm{C}$. Figure 9(a) shows that the thermal expansion of both the bcc particles and massive iron does not practically differ up to about $800^{\circ} \mathrm{C}$, then a sharp reduction of lattice constants occurs due to bcc $\rightarrow$ fcc transition. A similar reduction of length of iron rod was previously observed by the dilatation method at higher temperature (about $900^{\circ} \mathrm{C}$ ) [45]. Meanwhile, by X-ray diffraction technique Basinski et al. [46] have determined a phase transition point for a massive iron at $910^{\circ} \mathrm{C}$. The thermal expansion of the fcc particles shown in
Figure 9(b) significantly differs from that for a massive iron. With reducing temperature approximately down $700^{\circ} \mathrm{C}$, the reverse incomplete $\mathrm{fcc} \rightarrow$ bcc transition in particles takes place, albeit X-ray lines of the fcc lattice remain noticeable when reducing the temperature to about $560^{\circ} \mathrm{C}$. Even at temperature $20^{\circ} \mathrm{C}$ one succeeds in observing the very weak (111) fcc line offering the lattice constant being equal to 3.57 $\AA$. The same value of lattice constant has been found by extrapolation of the high-temperature data for ultrafine particles and a massive metal. The plot of temperature dependence of atomic volume (Figure 10) for fcc and bcc phases is more informative [43]. As seen in Figure 10, there would be a segregation of substance in the point of bcc $\rightarrow$ fcc particle transition; namely, the main part (according to the intensities of X-ray lines) turns into the fcc branch with a larger interatomic distance while the small part appears at the fcc branch of massive iron with smaller interatomic distance. Anti-Invar effect at ultrafine iron particles displays significantly stronger than in a massive metal. This confirms a comparison of data for the temperature-dependent atomic volume of the fcc lattice for particles with the calculated data for a massive substance taken from a work [47].

At $800^{\circ} \mathrm{C}$ Fe particles acquire the fcc structure, which transits into the bcc structure in the course of cooling. Therefore the single explanation of observed Mössbauer spectrum (Figure 8) can be in availability in the bcc lattice of regions which inherited a ferromagnetic ordering of spins of the high-temperature fcc Fe modification. It is the ferromagnetic state of the fcc structure which results in growth of lattice constant of particles compared to that of a bulk metal at high temperatures. As temperature goes down, a constriction of lattice constant to $a=3.57 \AA$ occurs at $23^{\circ} \mathrm{C}$, and a ferromagnetic state of the fcc structure simultaneously gradually transits in a paramagnetic state as demonstrated by the availability of a little singlet peak in the central part of Mössbauer spectrum (Figure 8). 


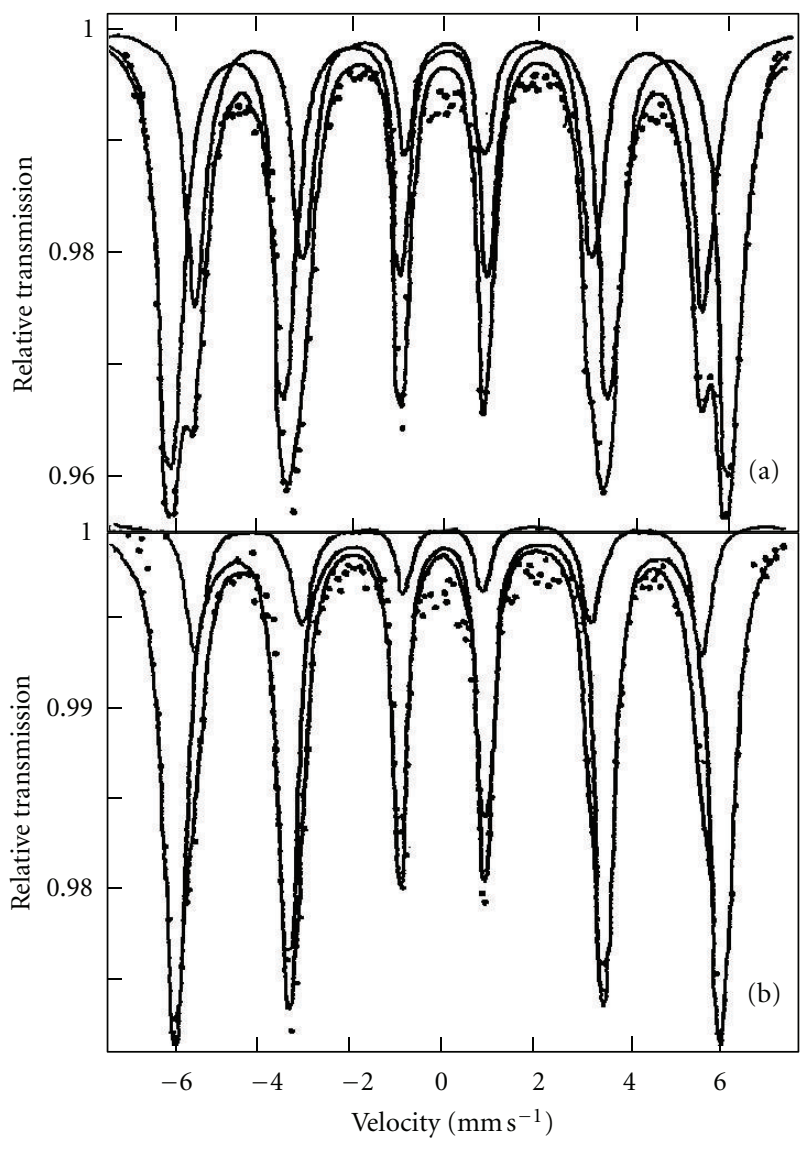

Figure 8: Mössbauer spectra of abnormal Fe particles of $\sim 50 \mathrm{~nm}$ size. (a) An initial powder; (b) the same powder after heating at $800^{\circ} \mathrm{C}$ for $1.5 \mathrm{~h}$ and subsequent quenching to room temperature.

4.3. Fe Nanoparticles and Its Oxides. When ageing the Fe powder in a vacuum chamber after its production for quite a short time, the primary iron monoxide $\mathrm{FeO}$ at the $\mathrm{Fe}$ particle shell transforms into $\mathrm{X}$-ray amorphous $\mathrm{Fe}_{3} \mathrm{O}_{4}$ oxide thick $\sim 2 \mathrm{~nm}$. The latter protects the particles against further oxidation in air for many years. However, by heating the powder in air $\mathrm{Fe}_{3} \mathrm{O}_{4}$ transforms to $\alpha-\mathrm{Fe}_{2} \mathrm{O}_{3}$. Annealing the Fe particles in vacuum results in crystallization of $\mathrm{Fe}_{3} \mathrm{O}_{4}$, accompanying with a formation of rather large magnetite crystallites, showing the well-expressed X-ray lines. The fact that these lines belong to magnetite rather than $\gamma$ - $\mathrm{Fe}_{2} \mathrm{O}_{3}$, which has a crystal lattice, very similar to that at $\mathrm{Fe}_{3} \mathrm{O}_{4}$, is evidenced by Figure 4, which presents the temperature dependences of ZFC and FC magnetization curves for both samples of Fe particles. A kink or even a jump at the ZFC curve for sample II at $T \cong 122 \mathrm{~K}$ clearly indicates Verwey transition occurring in $\mathrm{Fe}_{3} \mathrm{O}_{4}$ crystallites and being absent at $\mathrm{Fe}_{2} \mathrm{O}_{3}[18,20]$.

The kinks are seen on the temperature dependencies of $H_{C}$ and $M_{R}$ for sample II, which correspond to Verwey transition in the oxide shell of Fe particles. The change of coercivity and the hysteresis loop shift can be confronted with the crystallite size of oxide shell in Fe nanoparticles. The exchange interaction between the metallic core and the particle shell appears to be the largest when the oxide shell is amorphous. As the temperature increases, it goes down. At sufficiently high temperature, the coercivity diminishes at the expense of coarsening grains $\mathrm{Fe}_{3} \mathrm{O}_{4}$ reaching evidently the limiting value at about $500^{\circ} \mathrm{C}$. At temperatures above $560^{\circ} \mathrm{C}$ $\mathrm{Fe}_{3} \mathrm{O}_{4} \rightarrow \mathrm{FeO}$ transformation takes place.

The low (80 and $4.2 \mathrm{~K}$ ) temperature Mössbauer spectra of samples I and II (Figure 11) show, in addition to a sextet specific to $\alpha-\mathrm{Fe}$, also a sextet with broad lines corresponding to $\mathrm{Fe}_{3} \mathrm{O}_{4}$. In the room temperature spectrum of sample I there is a "sagging" line referred to a hyperfine field distribution (Figure 11). It disappears in 80 and $4.2 \mathrm{~K}$ spectra as well as after heating Fe powder (sample II) which is accompanied with an appearance of ferrimagnetic oxide $\mathrm{Fe}_{3} \mathrm{O}_{4}$. It is interesting that, for as-fresh powder (sample I) and that after cooling it down to $80 \mathrm{~K}$ followed by warming it to room temperature, there were no traces of oxide as a singlet or doublet in their Mössbauer spectra, as it would be expected in the case of superparamagnetic state of the particle oxide shell.

Small $\mathrm{Fe}_{3} \mathrm{O}_{4}$ crystallites in the primary X-ray amorphous oxide shell should be undoubtedly superparamagnetic at room temperature. Their magnetization vectors due to thermal fluctuations should undergo random changes of spatial orientation so that $\mathrm{Fe}_{3} \mathrm{O}_{4}$ crystallites on the average should be paramagnetic. At $80 \mathrm{~K}$ or lower temperatures, thermal fluctuations attenuate, and the crystallites become ferromagnetic. On the other hand, the room temperature Mössbauer spectrum of as-prepared powder (sample I, Figure 11(a)) shows that all cores of particles are ferromagnetic and, according to hysteresis loop measurements, should fix a stable ferrimagnetic oxide state. The absence of the ferrimagnetic oxide sextet in the room temperature Mössbauer spectrum occurs mainly due to a large thermal mean-square displacement of Fe nuclei relative to their equilibrium locations in oxide cluster. This results in a strong attenuation of Mössbauer effect probability. Thus, the core and crystallites of amorphous particle shell strongly magnetically interact with each other. With increasing the crystallite size of oxide, its magnetic interaction with the particle core attenuates. As a result, the hysteresis loop becomes narrower and its shift decreases (Figure 5(b)).

The room temperature Mössbauer spectrum of the heated sample II (Figure 11(b)) can be presented by superposition of two sextets related to $\alpha$-Fe and $\mathrm{Fe}_{3} \mathrm{O}_{4}$ as well as by a singlet belonging to the wustite $\mathrm{FeO}$, appearing at high-temperature decomposition of $\mathrm{Fe}_{3} \mathrm{O}_{4}$.

Based on a critical analysis of the available data and the own results, the authors [18] came to the following conclusions. The size of $\mathrm{Fe}_{3} \mathrm{O}_{4}$ crystallites in the oxide shell of Fe nanoparticles plays a crucial role in phenomenon of passivation and impact on magnetization of the metallic core. When the crystallites are small (X-ray amorphous oxide), antiferromagnetic arrangement of oxide spins is kept in a rather rigid order by the ferromagnetic core that results, in turn, in a strong exchange anisotropy and in a hysteresis loop shift. As crystallites grow and become isolated, their connection with the metallic core becomes weaker, the proper arrangement of spins is settled, and the hysteresis loop shift diminishes down to complete disappearance. 


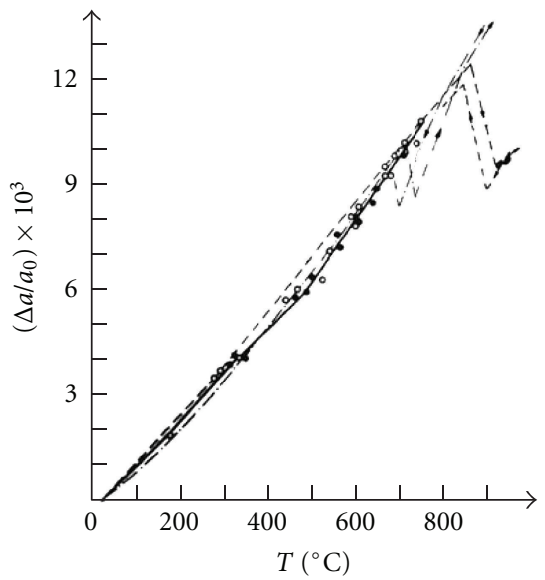

(a)

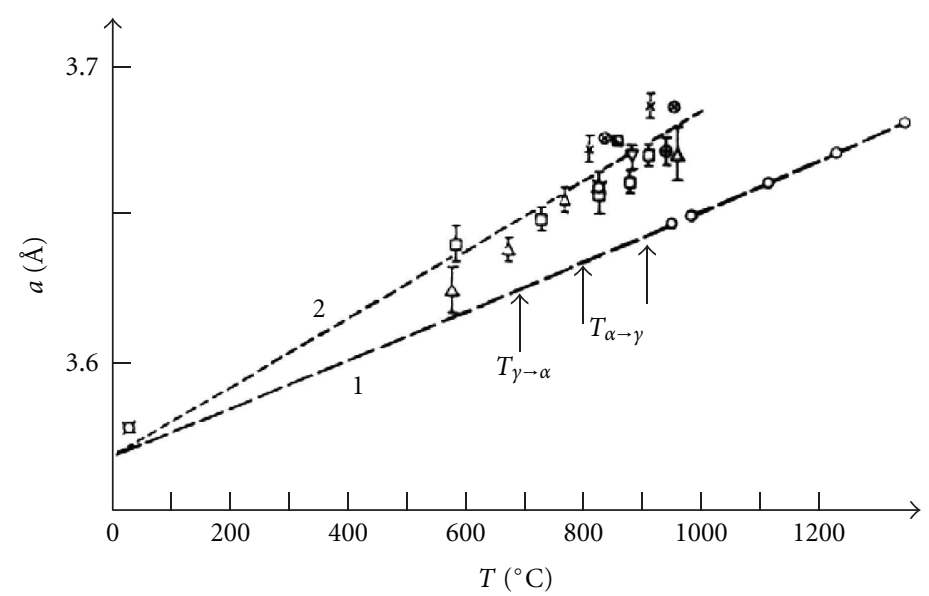

(b)

FIgURE 9: Thermal expansion of iron particles of $30 \mathrm{~nm}$ size. (a) $\alpha$-Fe. Signs are relative to aerosol particles; the dilatometric rod length measurements for pure Fe (solid line) and those for Fe with a 1.12\% C impurity (dashed line) [45]. (b) $\gamma$-Fe. Signs and the dotted line (2) refer to aerosol particles; the dashed line (1) presents the extrapolated data for a massive $\gamma$-Fe [46].

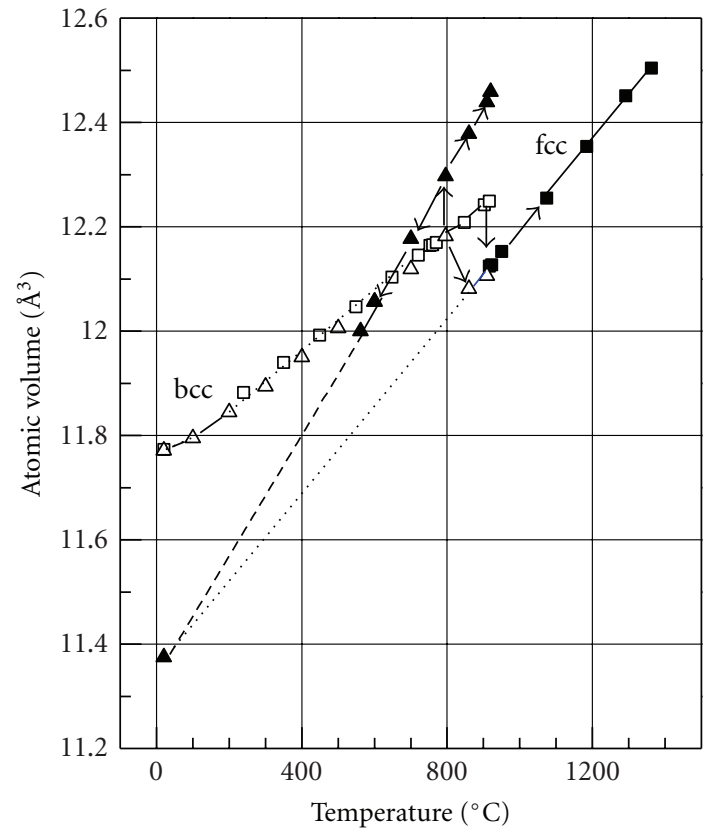

Figure 10: Temperature dependence of the atomic volume for Fe (30 nm particles and a bulk). $\Delta$ (bcc), $\boldsymbol{\Delta}$ (fcc)—particles [43]; $\square$ (bcc), $\mathbf{\square}$ (fcc)—bulk [46].

Mechanism of passivation in iron particles can be represented merely qualitatively [18]. Oxygen chemisorption of residual air in the setup takes place on a fresh surface of the aerosol particles, forming the primary oxide layer. Oxygen molecules adsorbed on this layer could reach the metallic core and oxidize it if they would be negative charged by capture of electrons. The latter may leave the metal by thermal electron emission or via tunnel mechanism. Due to loss of electrons the metal is positively charged and attracts the negative oxygen ions. If one supposes the primary amorphous oxide layer to be dielectric, creating a high potential barrier for electrons, then it may strongly hinder forming negativecharged oxygen molecules. Consequently, oxidation of particles covered with amorphous oxide may practically stop. Xray amorphous shell consisting of $\mathrm{Fe}_{3} \mathrm{O}_{4}$ clusters is evidently very compact and substantially connected with the metallic core, enveloping it elastically. Such a shell likely arises also at the early stage of bulk metal oxidation. However in this case it cannot exist sufficiently long because of breaks on surface's roughnesses and defects. Heating nanoparticles at a rather high temperature initiate crystallization of amorphous shell resulting in a loss of its homogeneity, compactness, and dielectric behavior. After such heat treatment, oxidation processes in the particles will run similar those in a bulk metal.

A direct experimental evidence of existence of the HS and LS states in the fcc lattice of Fe has been obtained so far only for relatively large iron particles. As it turned out, spin states of $\gamma$-Fe play a role in ultrafine particles of iron and Fe-rich FeMn [48-51], FeNi [51-54], and FePt [50, 55] binary alloys. Particles were prepared by evaporation of samples from tungsten wire in rare Ar atmosphere. In this case a rate of cooling aerosol particles is essentially slower than in the levitation-flow generator that does not allow one to freeze the HS state of the fcc Fe. At the same time the Mössbauer sextet of the bcc Fe particles of about $7 \mathrm{~nm}$ in diameter appears to be strongly "sagged" in the central part. A similar pattern was observed previously at aerosol particles of $13 \mathrm{~nm}$ in diameter in [56]. Such a "sagged" line often appeared in as-prepared FeNi, FeMn, and FePt powders, too.

\subsection{Structure and Magnetism of the Fe-Based Alloys Nanoparticles}

4.4.1. FeMn. FeMn (32 wt.\%) and FeMn (34.65 wt.\%) alloys have been investigated by authors in works [48-51]. X-ray lines of bcc, fcc, hcp FeMn phases as well as $\mathrm{Fe}_{3} \mathrm{O}_{4}$ and $\mathrm{MnO}$ 


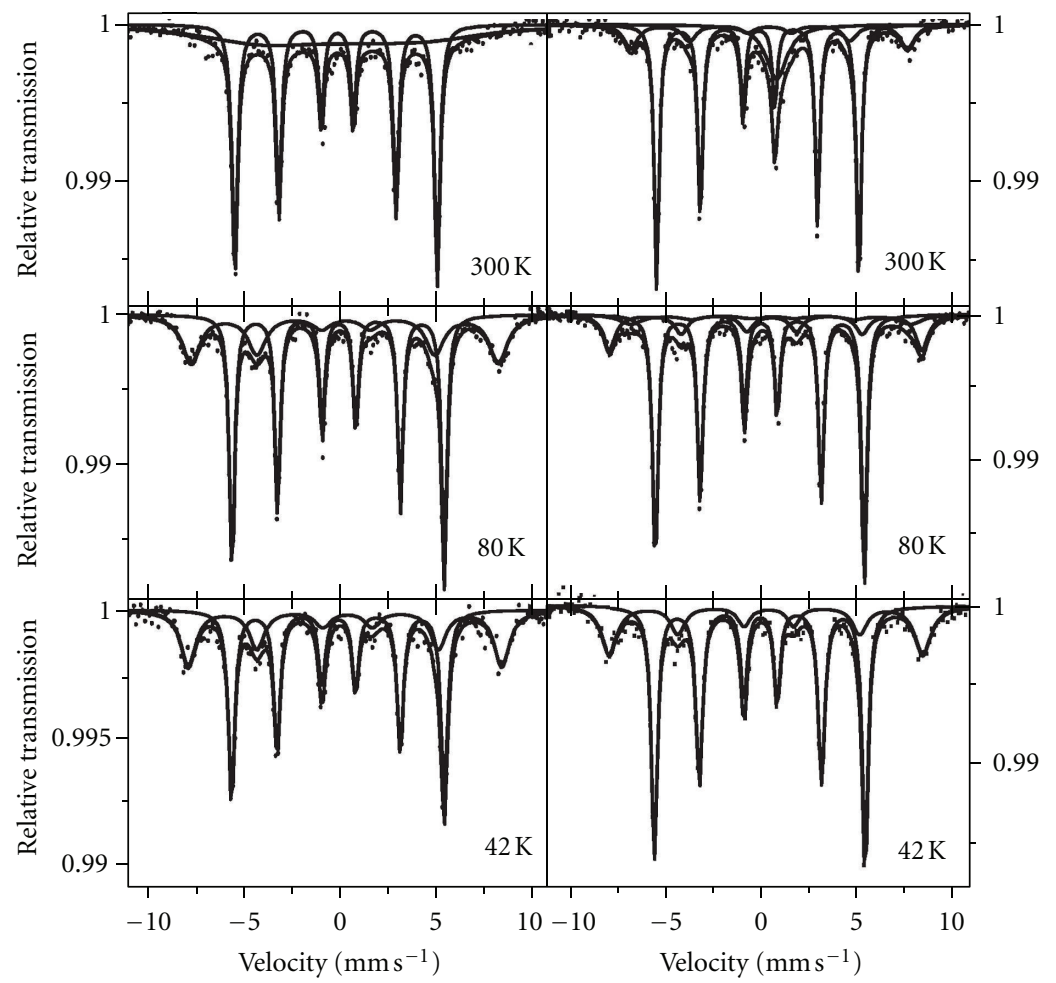

(a)

(b)

FIGURE 11: Mössbauer spectra of Fe nanoparticles taken at 300, 60, and 4.2 K. (a) Sample I; (b) sample II.

were observed. The small $(D=3$ and $7 \mathrm{~nm})$ particles show only two diffusive lines of a bcc FeMn and $\mathrm{Fe}_{3} \mathrm{O}_{4}$. A heating of powders for $2.5 \mathrm{~h}$ at $500^{\circ} \mathrm{C}$ has resulted in the growth of a bcc FeMn phase with a concurrent increase of both the $\mathrm{Fe}_{3} \mathrm{O}_{4}$ peak and an essentially less visible $\mathrm{MnO}$ peak. At the same time the hcp FeMn phase arises by martensite transformation of the fcc phase at its cooling, and a thickness of oxide shell grows of 2 to $4 \mathrm{~nm}$ though the core size does not practically change [48]. Note that the FeMn (34.65\%) foil shows mainly the fcc phase with an admixture of the hcp FeMn phase instead of bcc FeMn phase specific even to large particles $(D$ $=15 \mathrm{~nm})$.

Mössbauer spectra of FeMn (32\%) and FeMn (34.65\%) nanoparticles are given in Figure 12. They are similar to spectra for the alloys of both compositions. The area ratio of the singlet to the sextet depends on the pressing power while cleaning the beaker walls and crumbling the sample in an agate mortar before the Mössbauer studies. The spectrum shown in Figure 12(b) can be observed for both small $(D=$ $3 \mathrm{~nm})$ and large $(D=7 \mathrm{~nm})$ as-prepared particles. A sextet in Figure 12(b) can be decomposed into two components with $H_{i 1}=35.0 \mathrm{~T}$ and $H_{i 2}=31.0 \mathrm{~T}$ which gives an average value $H_{i}=33.0 \mathrm{~T}$ as at a massive iron. After heat treatment of the particles $(D=7 \mathrm{~nm})$ the average field $\left(H_{i}=\right.$ $34.0 \mathrm{~T}$ ) and the singlet increases (Figure $12(\mathrm{~d})$ ). It means that some fraction of the component with the field $H_{i 2}$ became superparamagnetic.

A central peak in the Mössbauer spectra of small $(D=3 \mathrm{~nm})$ FeMn $(34.65 \%)$ particles at room temperature
(Figure 12(b)) contains a singlet referred to the hcp phase and two doublets, one of which is referred to the fcc phase of the FeMn, and the other one having a large isomer shift $\left(0.38 \mathrm{~mm} \mathrm{~s}^{-1}\right.$ relative to Armco iron) belongs to the iron oxide [49]. The Mössbauer spectra of these particles heated at $300^{\circ} \mathrm{C}$ for $0.5 \mathrm{~h}$ show, apart from six lines of the bcc FeMn (two well-defined components with fields $H_{i 1}=35.0 \mathrm{~T}$ and $H_{i 2}=31.0 \mathrm{~T}$ of about equal area in the spectrum) and a strong singlet peak, three sextets with $H_{i}=48.8 \mathrm{~T}$ and $46.1 \mathrm{~T}$ $\left(\mathrm{Fe}_{3} \mathrm{O}_{4}\right)$, and $H_{i}=51.7 \mathrm{~T}\left(\alpha-\mathrm{Fe}_{2} \mathrm{O}_{3}\right)$ [49]. Depending on some change of experiment conditions in different tests and a place of taking-off powder the relation of bcc and hcp phases somewhat varies. At that the value $H_{i 2}$ keeps within the range $(30.4 \div 31.0) \mathrm{T}$ whereas the field $H_{i 1}$ can be higher the field $H_{i}=33.0 \mathrm{~T}$ by the value of zero to $5.0 \mathrm{~T}$. When decreasing the temperature to $4 \mathrm{~K}$, the superparamagnetic oxide transforms to the ferromagnetic phase with a hyperfine field about $49.7 \mathrm{~T}$, the singlet is unaffected, and the second doublet is broadened and splits [51]. At the same time the fields $H_{i 1}$ and $H_{i 2}$ can grow, for example, $H_{i 1}$ of 33.0 to $34.32 \mathrm{~T}$ and $H_{i 2}$ of 30.4 to $31.9 \mathrm{~T}$, specifying as HS and LS states change.

Thus, unlike the massive FeMn (32-35\%) alloys having a metastable austenite fcc structure at room temperature [57, 58 ] the fine particles of the same alloys formed and rapidly quenched in an inert gas atmosphere acquire a stable bcc structure admixed with the intermediate hcp phase due to the martensite transition of a high-temperature fcc austenite. As-fresh small FeMn (32-35\%) particles possessing a bcc 
structure likely have simultaneously both HS and LS states in their lattice, the relation of which changes with temperature and particle composition. LS state is evidently more stable than HS state at reduced temperatures whereas HS state in small fcc particles probably prevails at high temperatures. When the temperature decreases, this state transforms into a low spin paramagnetic (or antiferromagnetic) one of either the same fcc phase or the hcp phase formed from the fcc phase of the FeMn alloy. It is supposed that the nature of a strong "sagging" of the central part of Mössbauer spectra is caused by continuous distribution of hyperfine fields at $\mathrm{Fe}$ nuclei which is connected with decomposition of the HS state of the fcc Fe. The detailed discussion of the results for aerosol FeMn particles has been presented in [43, 49-51].

4.4.2. FeNi. In comparison with FeMn system, an analysis of the results obtained for ultrafine FeNi (30.3, 35, and 52\%) particles turns out to be significantly simpler because of lacking the intermediate hcp phase $[43,51-54]$. The lattice constants of the bcc phase at even the smallest $(D=5-8 \mathrm{~nm})$ particles are very close to that of pure bcc Fe. The FeNi (52\%) alloy preserves its fcc structure regardless of the particle size. On the other hand, FeNi (30.3 and 35\%) alloys in the bulk state have a fcc structure with the slight admixture of bcc phase, while the particles with a core size of $5-8 \mathrm{~nm}$ consist exclusively of the bcc phase. As these particles become larger, the bcc $\rightarrow$ fcc transformation takes place which is completed in FeNi (35\%) particles having $D=9 \mathrm{~nm}$, although the larger $(15 \mathrm{~nm})$ FeNi $(30.3 \%)$ particles have an appreciable bcc phase impurity. Such behavior does not practically change after heating the particles at $300^{\circ} \mathrm{C}$ for $5 \mathrm{~h}$ in vacuum and subsequent keeping for $0.5 \mathrm{~h}$ in liquid nitrogen. Meanwhile, the complete bcc $\rightarrow$ fcc transformation occurs when small FeNi (30.3\%) and FeNi (35\%) particles have been held in Ar for $0.5 \mathrm{~h}$ at 820 and $900^{\circ} \mathrm{C}$, respectively, followed by quenching to room temperature.

The typical Mössbauer spectra of FeNi (30.3\%) foil and nanoparticles are shown in Figure 13. It is seen a strong change of the spectrum shape on the particles size. However, in the case of FeNi (52\%) alloy it holds in going from a bulk to $7 \mathrm{~nm}$ particles. Meanwhile, the Mössbauer spectra of FeNi (30.3\%) and FeNi (35\%) particles with $D=5-8 \mathrm{~nm}$, having the bcc structure, as opposed to the spectra of the initial foils, show sextets with rather broad lines and a small doublet due to Fe oxides (Figure 13(c)). The sextets can be decomposed into two components corresponding to the hyperfine fields $H_{1}=34.5 \mathrm{~T}$ and $H_{2}=31.8 \mathrm{~T}$. As a result of quenching the smallest FeNi (30.3\%) and FeNi (35\%) particles from a temperature above $800^{\circ} \mathrm{C}$, the sextet with $\mathrm{H}_{2}=31.8 \mathrm{~T}$ in the spectrum is kept while the sextet with $H_{1}=34.5 \mathrm{~T}$ passes into the central line (Figure 13(d)). We can ascribe the sextets with the hyperfine fields $H_{1}=34.5 \mathrm{~T}$ and $H_{2}=31.8 \mathrm{~T}$ to a remnant of the magnetic order of the HS and LS states of the hightemperature fcc alloy modification in the bcc lattice of the particles $[43,51]$. In accordance with the X-ray data, the singlet can be related to the fcc phase and the sextet to the bcc phase. At $T=298 \mathrm{~K}$ the "sagged" line reflects the

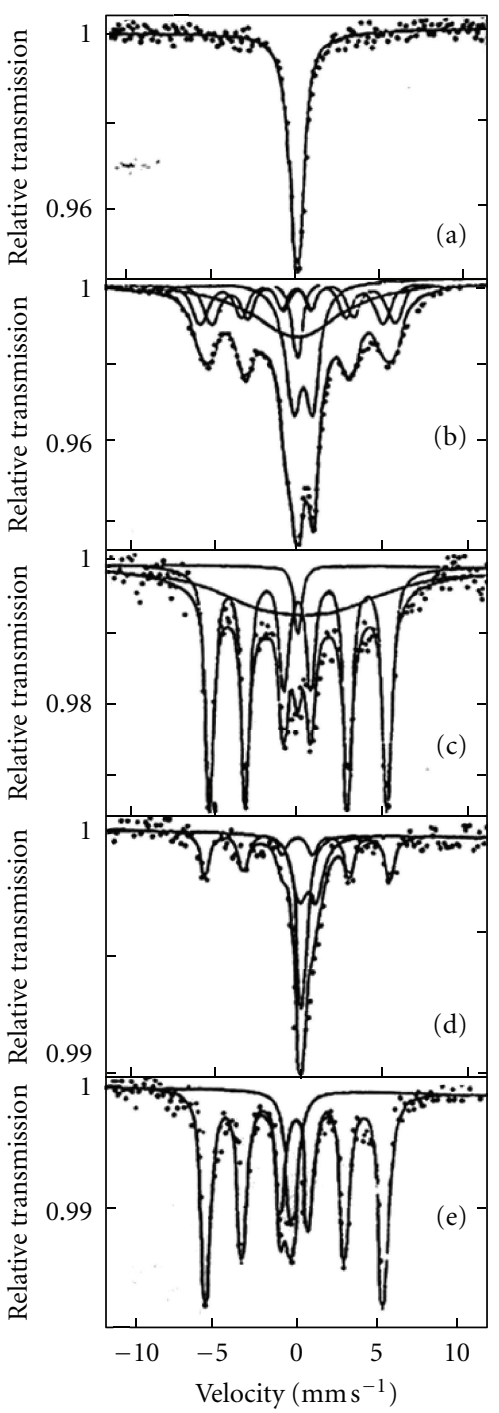

Figure 12: Mössbauer spectra for FeMn (32\% and 34.65\%) nanoparticles. (a) Small FeMn (32\%) particles with the core size of $D=3 \mathrm{~nm}$; (b) small FeMn (34.65\%) particles with the core size of $D=3 \mathrm{~nm}$; relative areas $(S)$ of spectrum components: singlet, $S$ $=0.06$; doublet, $S=0.25$; two sextets: (I), $S=0.17$, hyperfine field $H_{i 1}=35.0 \mathrm{~T}$; (II), $S=0.17, H_{i 2}=31.0 \mathrm{~T}$; "sagged" line, $S=0.35$; (c) FeMn $(34.65 \%)$ particles with the core size of $D=7 \mathrm{~nm}$ crumbled in an agate mortar; singlet, $S=0.03$; sextet $S=0.56, H_{i}=35.0 \mathrm{~T}$; "sagged" line, $S=0.35$; (d) FeMn $(34.65 \%)$ particles with the core size of $D=7 \mathrm{~nm}$ after heating in vacuum at $500^{\circ} \mathrm{C}$ for $1 \mathrm{~h}$; singlet, $S=0.30$, doublet, $S=0.35$; sextet $S=0.35, H_{i}=34.0 \mathrm{~T}$; (e) FeMn (34.65\%) particles with the core size of $D=15 \mathrm{~nm}$; singlet, $S=0.14$; sextet $S=0.86, H_{i}=32.9 \mathrm{~T}$.

distribution of hyperfine fields in the sample and the split doublet is apparently caused with superparamagnetic (SP) X-ray amorphous Fe oxides. At $T=77 \mathrm{~K}$ the "sagged" line disappears, the $\mathrm{Fe}$ oxide transforms from SP to a ferromagnetic (FM) state, displaying two sextets. These sextets are referred to the hyperfine fields 42.5 and $48.2 \mathrm{~T}$ with accordance to octahedral and tetrahedral sites in the spinel $\mathrm{Fe}_{3} \mathrm{O}_{4}$, respectively [59]. The area of these sextets 


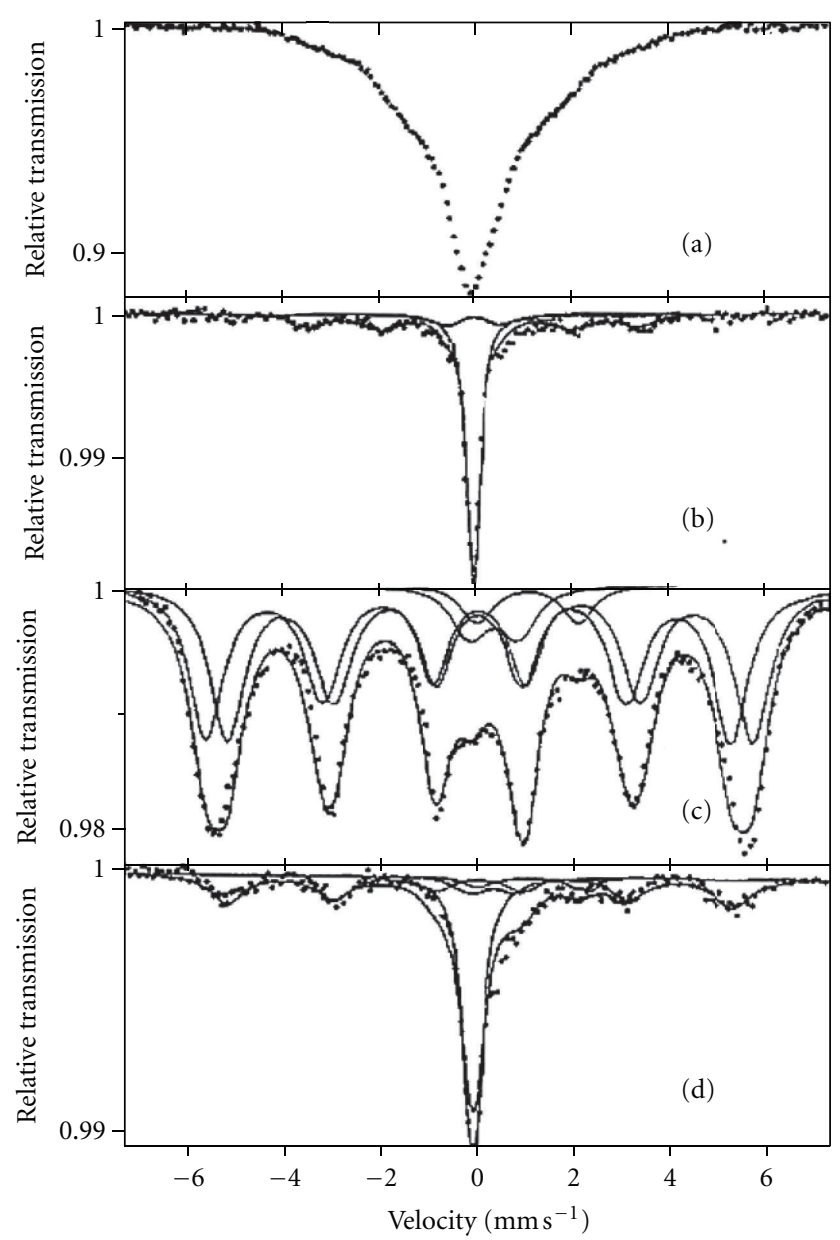

Figure 13: Mössbauer spectra for FeNi massive alloy (foil) and nanoparticles. (a) FeNi (30.3\%) foil; (b) FeNi (30.3\%) particles with a size of $D=15 \mathrm{~nm}$; singlet, $S=0.57$; sextet, $S=0.43$, $H_{i}=33.0 \mathrm{~T}$; (c) FeNi (35\%) particles with a size of $D=8 \mathrm{~nm}$; two oxide doublets, $S_{\Sigma}=0.12$; two sextets: (I) $S_{1}=0.44, H_{i 1}=34.5 \mathrm{~T}$; (II) $S_{2}=0.44, H_{i 2}=31.8 \mathrm{~T}$; (d) FeNi (35\%) particles with a size of $D=8 \mathrm{~nm}$ heated at $900^{\circ} \mathrm{C}$ for $30 \mathrm{~min}$ followed by quenching to room temperature; singlet, $S=0.42$; two oxide doublets, $S_{\Sigma}=0.18$; sextet, $S=0.43, H_{i}=31.8 \mathrm{~T}$.

reflects the oxide content in FeNi (30.3\%) particles. When the temperature falls down to $4 \mathrm{~K}$, the singlet transforms to a wide peak described by a sextet with $H_{i}=3.3 \mathrm{~T}$, and FM Fe oxide is presented, for simplicity, by one sextet with $H_{i}=49.6 \mathrm{~T}$. The sextets related to the bcc phase can be decomposed into two components with the hyperfine fields $H_{1}=36 \mathrm{~T}$ and $H_{2}=33.2 \mathrm{~T}$ which somewhat increase with decreasing the temperature. These fields can be attributed to a remnant of HS and LS states of the fcc phase in a bcc lattice. Broadening of the singlet at $4 \mathrm{~K}$ is evidently caused by $\mathrm{PM} \rightarrow \mathrm{AF}$ (antiferromagnetic) transition of the fcc phase.

4.4.3. FeNiMn. Since massive fcc FeMn alloys are antiferromagnetic and FeNi alloys ferromagnetic, it is of certain interest to follow what influence has the sequential substitution

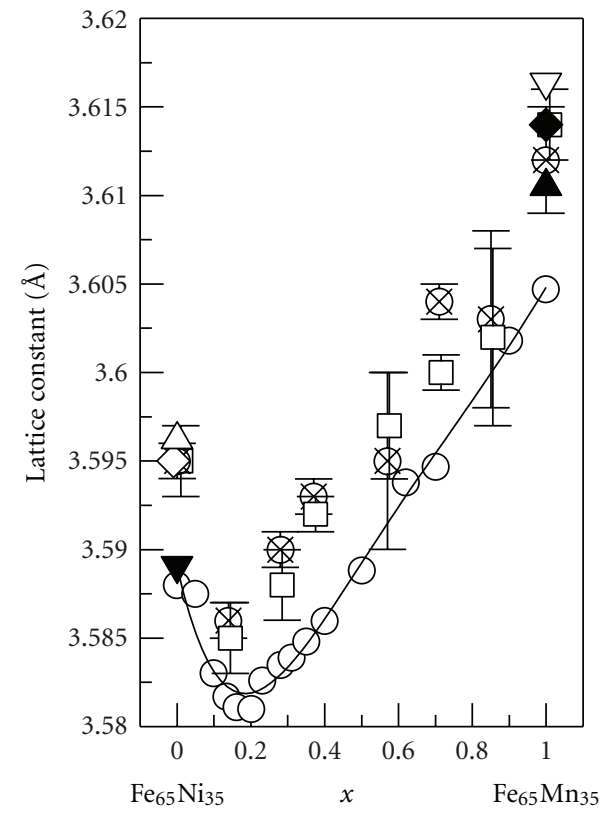

FIguRE 14: Concentration dependence of the $\mathrm{Fe}_{65}\left(\mathrm{Ni}_{1-x} \mathrm{Mn}_{x}\right)_{35}$ bulk alloy lattice parameter, $a$, at room temperature. $(\otimes)$ initial foil; ( $\square$ ) foil after heating at $900^{\circ} \mathrm{C}$ for $2 \mathrm{~h}$; (O) data of [62]; $(\triangle)$ $\mathrm{Fe}_{62.4} \mathrm{Ni}_{37.6} ;(\diamond) \mathrm{Fe}_{65} \mathrm{Ni}_{35},(\boldsymbol{\nabla}) \mathrm{Fe}_{67.4} \mathrm{Ni}_{32.6}$, interpolated data from [63]; (\) $\mathrm{Fe}_{62.4} \mathrm{Ni}_{37.6} ;(\diamond) \mathrm{Fe}_{65} \mathrm{Ni}_{35} ;(\nabla) \mathrm{Fe}_{67.4} \mathrm{Ni}_{32.6}$, interpolated data from [64].

of Ni by Mn in ternary FeNiMn alloy with approximately constant Fe content of about 65\% in both massive and highdispersed samples $[43,60,61]$. The alloy composition can be written as $\mathrm{Fe}_{65}\left(\mathrm{Ni}_{1-x} \mathrm{Mn}_{x}\right)_{35}(0 \leq x \leq 1)$ having in mind that a deviation of $\mathrm{Fe}$ content from $65 \%$ does not exceed $4 \%$ in individual samples. It is seen in Figure 14 that a general behaviour of concentration dependence for fcc lattice constants of alloys under study looks like that obtained by Shiga [62]. However, our data are somewhat above this curve, and they agree rather well with the data of other works for limiting binary FeMn (34.65\%) [63] and FeNi (35\%) [64] alloys. Undoubtedly, there is some relation between the lattice constant and magnetism for FeNiMn alloy that confirms a similar character of concentration dependence for magnetic moment and lattice constant (see, e.g., Figure 2 in [38]). A question is in what is primary. In the case of binary transition metal alloys a deviation from the Vegard's rule establishing a linear lattice constant dependence upon the alloy composition is ascribed to influence of the magnetic state of substance [65]. It is well known that with reducing $\mathrm{Ni}$ content in the FeNi alloy, its magnetic moment and lattice constant first increase proportionally and then sharply fall down nearly FeNi (30\%) composition (see, e.g., [38]). Weiss [41] explained this phenomenon by the fact that at $\mathrm{Ni}$ content higher than $30 \%$ the ground state is ferromagnetic, and at lower Ni content it is antiferromagnetic. Therefore as temperature increases or $\mathrm{Ni}$ content reduces in alloys with composition close to $\mathrm{FeNi}(30 \%)$, a sharp reduction of their volume and magnetic moment should occur. Weiss supposed 
that there is a similar picture in fcc FeMn alloys, but at room temperature the ground state for them is antiferromagnetic, and this state should be gradually changed by a ferromagnetic one as $\mathrm{Mn}$ content reduces or temperature rises. This should result in increasing magnetization and thermal expansion. In fact, the Fe-rich FeMn alloys in a definite temperature range are actually Invar ones and they show reduction of thermal expansion coefficient [66]. Only in a paramagnetic state at high temperatures the thermal expansion of alloys sharply increases (anti-Invar effect) [58].

In our opinion, a substitution of $\mathrm{Ni}$ by $\mathrm{Mn}$ in initial $\mathrm{Fe}_{65} \mathrm{Ni}_{35}$ system originating a ternary $\mathrm{Fe}_{65}\left(\mathrm{Ni}_{1-x} \mathrm{Mn}_{x}\right)_{35}$ alloy should result mainly in reducing the magnetic moment and lattice constant owing to the Mn inherent LS state with less values of the magnetic moment and lattice constant. A presence of Mn noticeably enhances a drop of the hyperfine field at Fe nuclei in the ternary alloy [63]. On the other hand, a substitution of $\mathrm{Mn}$ by $\mathrm{Ni}$ in the FeMn (35\%) originating a $\mathrm{Fe}_{65}\left(\mathrm{Ni}_{1-x} \mathrm{Mn}_{x}\right)_{35}$ alloy should be accompanied by increasing its volume and magnetic moment at the expense of arising HS state of Ni instead of the LS state of Mn. It is just such a picture which was observed in Shiga's work [62]. Unlike his results the ternary alloys of every composition in our experiments appeared paramagnetic at room temperature. Probably, this discrepancy of data for Neel and Curie points is caused with some variation of Fe content in alloys.

Unlike the massive ternary $\mathrm{Fe}_{65}\left(\mathrm{Ni}_{1-x} \mathrm{Mn}_{x}\right)_{35}$ alloys having the fcc structure, the aerosol $(5-8 \mathrm{~nm})$ particles produced from them had the bcc structure with a slight fcc admixture. The X-ray lines intensity of the latter enlarged as the particle size grew up to $10-15 \mathrm{~nm}$ or Ni content increased. The typical Mössbauer spectrum of ultrafine Fe (64.94\%) Ni $(25.25 \%)$ $\mathrm{Mn}(9.83 \%)$ particles with 5-8 $\mathrm{nm}$ and $10-15 \mathrm{~nm}$ in diameter are shown in Figure 15(a) and Figure 15(b), respectively. In accordance with the structural data, a singlet can be related to the paramagnetic fcc phase, sextet to the bcc phase, and doublet to Fe oxides. The absence of a powerful central peak in the Mössbauer spectrum of the smallest particles (Figure 15(a)) indicates a suppression of superparamagnetic effect at the expense of compacting powder. A "sagging" of sextet was eliminated by heating the particles in vacuum at temperature $\geq 300^{\circ} \mathrm{C}$ for $0.5 \mathrm{~h}$. The sextet itself can be decomposed into two components with hyperfine fields $H_{1}=34.5 \mathrm{~T}$ and $H_{2}=29.5 \mathrm{~T}$. Just as in the case of binary FeNi and FeMn alloys, we ascribe the hyperfine fields $H_{1}=$ $34.5 \mathrm{~T}$ and $H_{2}=29.5 \mathrm{~T}$ to a remnant states of the hightemperature fcc phase in the bcc structure. The observed paramagnetic fcc phase possesses the LS state.

4.4.4. $\mathrm{Fe}_{3} \mathrm{Pt}$. $\mathrm{Fe}_{3} \mathrm{Pt}$ alloy belongs to a group of so-called Invar alloys possessing anomalous physical properties. The phase diagram of $\mathrm{Fe}_{3} \mathrm{Pt}$ alloy is similar to that for the FeNi system $[67,68]$. As the $\mathrm{Pt}$ content increases, the starting point for the $\gamma$ (fcc) $\rightarrow \alpha$ (bcc) martensite transition drops from $910^{\circ} \mathrm{C}$ for pure $\mathrm{Fe}$ down to room temperature for an alloy containing about 20 at.\% Pt. The reverse $\alpha \rightarrow \gamma$ transition takes place with temperature hysteresis and completes close

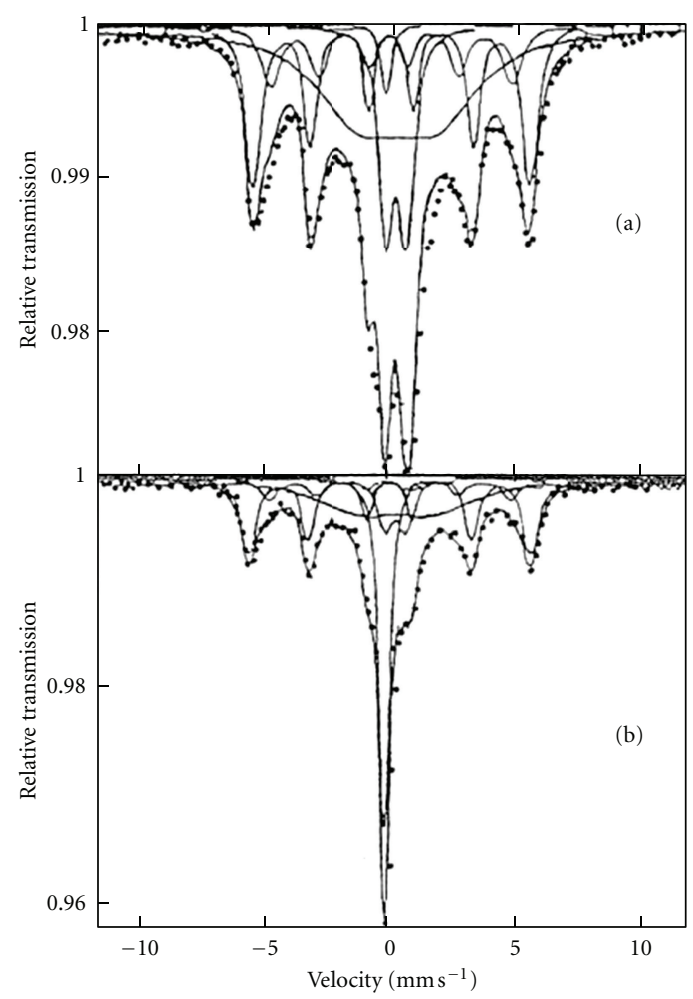

Figure 15: Mössbauer spectra of Fe (64.94\%) Ni (25.25\%) Mn $(9,83 \%)$ nanoparticles. (a) Nanoparticles with a core size of $D=$ (5-8) nm; singlet, $S_{1}=0.02$; oxide doublet, $S_{2}=0.16$; two sextets: (I) $S_{3}=0.25, H_{3}=34.0 \mathrm{~T}$; $S_{4}=0.13, H_{4}=29.5 \mathrm{~T}$; (II) "sagged" line $S_{5}=0.44$. (b) Nanoparticles with a core size of $D=(10-15) \mathrm{nm}$; singlet, $S_{1}=0.12$; oxide doublet, $S_{2}=0.12$; two sextets: (I) $S_{3}=0.24$, $H_{3}=34.5 \mathrm{~T}$; (II) $S_{4}=0.13, H_{4}=29.5 \mathrm{~T}$; "sagged" line $S_{5}=0.39$.

to $600^{\circ} \mathrm{C}$ at the $\mathrm{Pt}$ concentration corresponding to the $\mathrm{Fe}_{3} \mathrm{Pt}$ composition. Alloys with larger $\mathrm{Pt}$ content have a fcc structure even when the temperature falls down to $77 \mathrm{~K}$. The curves of concentration dependence for saturation magnetization and also for the thermal expansion of the alloy sharply fall down to near zero in the temperature range $(20-70)^{\circ} \mathrm{C}$. The same sharp drop of the coefficient of thermal expansion was observed previously in the fcc phase of the FeNi alloy containing about 30\% Ni [69]. It is very surprising that the coefficient of thermal expansion and saturation magnetization hold their values in a certain range of varying the additive to iron after drop in the concentration-dependent curves (Invar effect) even though a change of crystalline structure takes place in this case. The most acceptable explanation of the effect is suggested by the two-state Weiss' model [41]. However, phase transitions and phenomena of phase ordering can complicate the pattern considerably. $\mathrm{Fe}_{3} \mathrm{Pt}$ alloy offers a unique possibility to unambiguously distinguish between the crystallographic and magnetic ordering.

Unlike FeNi and FeMn alloy, $\mathrm{Fe}_{3} \mathrm{Pt}$ system is noted by a significant difference of atomic scattering factors of components. This permits to study ordering processes in alloy by 


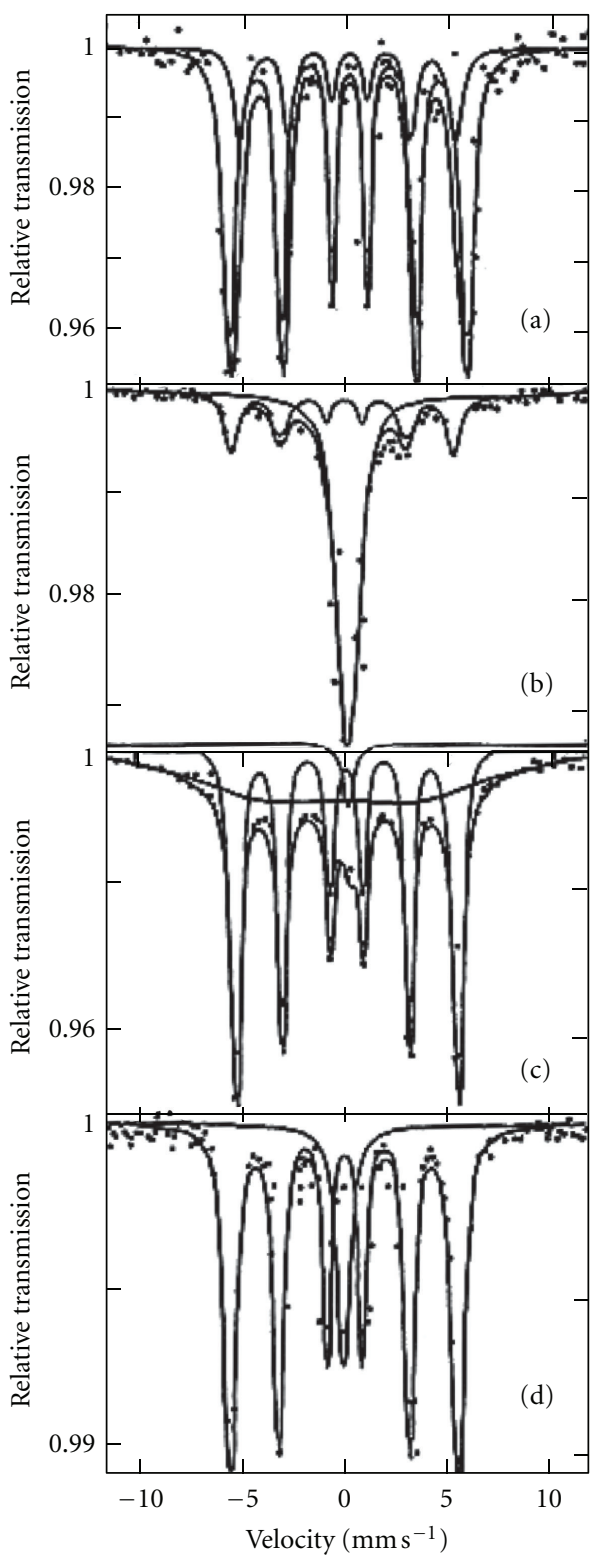

Figure 16: Mössbauer spectra of $\mathrm{Fe}_{3}$ Pt alloy. (a) bcc foil thick $4 \mu \mathrm{m}$; two sextets: (I) $S_{1}=0.75, H_{1}=35.3 \mathrm{~T}$; (II) $S_{1}=0.25, H_{1}=32.3 \mathrm{~T}$; (b) bcc particles of $7 \mathrm{~nm}$ size; singlet $S_{1}=0.69$; sextet, $S_{1}=0.31$, $H_{1}=33.3 \mathrm{~T}$; (c) particles of $20 \mathrm{~nm}$ size; singlet $S_{1}=0.02$; sextet, $S_{2}=0.44, H_{1}=33.3 \mathrm{~T}$; "sagged" line $S_{3}=0.54$; (d) the same powder after heating at $650^{\circ} \mathrm{C}$ in vacuum $2.7 \times 10^{-2} \mathrm{~Pa}$ for $2 \mathrm{~h}$; singlet $S_{1}=0.15$; sextet, $S_{1}=0.85, H_{1}=33.7 \mathrm{~T}$.

structural methods because additional superstructure lines arise in the ordered structure [67].

A thin $\mathrm{FePt}$ (24.52 at.\%) foil with iron enriched in the isotope ${ }^{57} \mathrm{Fe}$ to $19 \%$ and the impurity content no higher than $0.05 \%$ was used as a starting material in our experiments $[50,55,70]$. This composition is close enough to the formula $\mathrm{Fe}_{3} \mathrm{Pt}$. The foil was kept before in air for more than 15 years. It had the bcc structure without any traces of the fcc phase and showed a sextet lines in its Mössbauer spectrum (Figure 16(a)). Because the sextet peaks were rather broad, they could be decomposed into two components, namely, the predominant component with the hyperfine field $H_{1}=$ $35.0 \mathrm{~T}$ and the weaker one with $\mathrm{H}_{2}=32.3 \mathrm{~T}$. By additional experiments with $\mathrm{FePt}(24.52$ at.\%) foil it has been shown that the dominant sextet with $H_{1}$ could be assigned to HS state of the fcc phase whereas the weaker sextet with $\mathrm{H}_{2}$ to LS state of the fcc phase. After a prolonged storage of the foil in air (in our case ageing was for 15 years), the fcc phase completely transformed to the bcc phase. The latter inherits side by side mainly the HS state and a slight fraction of the LS state of the former fcc structure. Mössbauer spectra of the ordered $\mathrm{Fe}_{3} \mathrm{Pt}$ alloy prepared by slow cooling of the alloy samples from a high temperature are given in Figure 17 [70]. The specific relationship of the peak intensities and the reduced effective magnetic field at Fe nuclei compared with the disordered structure, as well as the lack of the central peak in the spectrum, are characteristic for the ordered ferromagnetic state of the fcc $\mathrm{Fe}_{3} \mathrm{Pt}$.

The aerosol $\mathrm{Fe}_{3} \mathrm{Pt}$ particles with a size of about $7 \mathrm{~nm}$ have a disordered bcc structure $[43,55]$. The corresponding Mössbauer spectrum is shown in Figure 16(b). After heating the sample at $500^{\circ} \mathrm{C}$ in vacuum for $1 \mathrm{~h}$ the shape of the spectrum and the structure remains practically unchanged. Because of absence of the fcc phase in the sample which could be paramagnetic, the central peak in Figure 16(b) should be ascribed to exhibition of superparamagnetism of particles weakly linked to each other. The larger particles of about $20 \mathrm{~nm}$ size show, in along with the main bcc phase, an admixture of the fcc phase. In this case the Mössbauer spectrum (Figure 16(c)) can be decomposed into a sagged line, sextet, and a small central singlet. Heating of the same powder at $650^{\circ} \mathrm{C}$ in vacuum for $2 \mathrm{~h}$ gave rise to changing the spectrum, namely, increasing the area of the singlet line at the expense of the disappearance of the spectrum sagging (Figure 16(d)). The singlet to sextet ratio has increased approximately in 4 times. Meanwhile, according to the X-ray diffraction patterns, the fcc phase fraction does not increase after heating the sample, but even somewhat decreases. These results can be interpreted as a transformation of distribution of high-temperature HS states of the fcc in the bcc lattice into a paramagnetic LS state. A corresponding LS hyperfine field $H_{2}=32.3 \mathrm{~T}$ in the bcc phase is close to the value $H=33.0 \mathrm{~T}$ for iron. The sextet component of the foil with the high hyperfine field $H_{1}=35.3 \mathrm{~T}$ lacks in the Mössbauer spectra of ultrafine particles. Thus, in the case of $\mathrm{Fe}_{3} \mathrm{Pt}$ system the HS and LS states of the fcc phase display themselves more distinctly in the massive alloy than in ultrafine particles.

4.4.5. FeCo. FeCo alloy has a stable bcc structure in a wide Co content range from 0 to 73 at.\% and the fcc structure for $80-95$ at.\% Co content $[67,68]$. Therefore, one cannot expect manifestation of the Invar anomalies connected with the presence of two-spin stases in the Fe-rich fcc phase. In our experiments [71] an attempt was undertaken to freeze the high-temperature fcc modification of the FeCo alloy and its magnetic ordering by employing a rapid cooling of aerosol particles in the course of their preparation by the gas evaporation technique. 


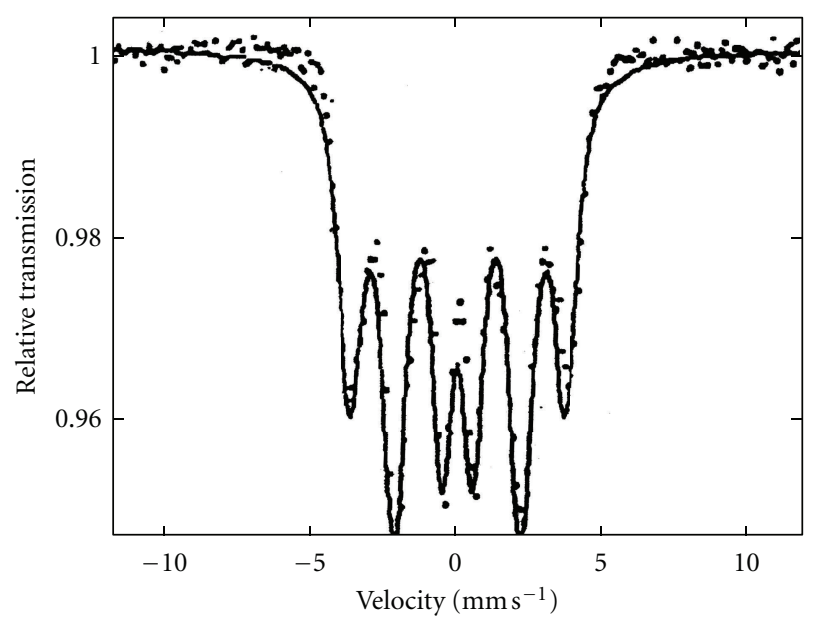

(a)

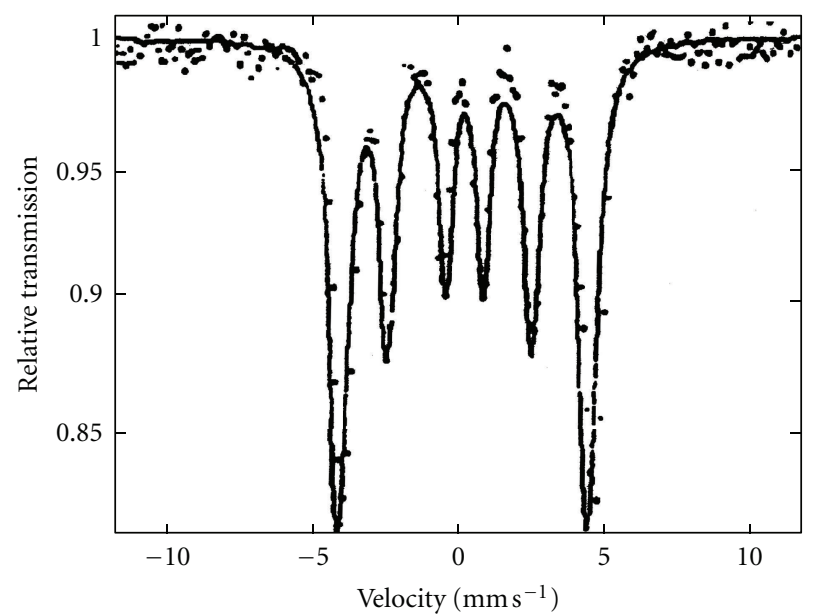

(b)

FIgure 17: Mössbauer spectra of ordered $\mathrm{Fe}_{3} \mathrm{Pt}$ alloy. (Cooling rate of the high temperature heating is about $1^{\circ} \mathrm{C} \times$ min ${ }^{-1}$ ). (a) Foil with completely ordered fcc structure obtained after annealing at $640^{\circ} \mathrm{C}$ for $48 \mathrm{~h}$ followed by cooling to room temperature. The spectrum consists of a sextet with $H=22.6 \mathrm{~T}$; (b) spectrum of the foil after heating at $1100^{\circ} \mathrm{C}$ for $4 \mathrm{~h}$ followed by cooling to room temperature. The ordered fcc phase with a noticeable fraction of the bcc phase. Both structures are deformed. The spectrum consists of a sextet with $H=26.3 \mathrm{~T}$.

The aerosol FeCo (10-95\%) particles with a size of about 10-15 $\mathrm{nm}$ were studied. It was shown that the structure and lattice parameters with an accuracy of $\pm 0.002 \AA$ did not differ for the particles and bulk alloys. All particles contained only one phase (bcc for FeCo $(<80 \%)$ and fcc for FeCo $(>90 \%))$ except for FeCo (35\%) system which had in some samples, in addition to the bcc phase, also a slight admixture of the fcc one. Availability of the fcc phase was reflected by a singlet in the Mössbauer spectrum (Figure 18(a)). By heating the $\mathrm{FeCo}(35 \%)$ particles at $600^{\circ} \mathrm{C}$ for $0.5 \mathrm{~h}$ in vacuum 2.7 $\times 10^{-3} \mathrm{~Pa}$ a "sagged" line disappeared, and a fraction of the sextet belonged to the bcc phase increased and attained $66 \%$. This allowed one to evaluate the Fe oxide content which was less than $34 \%$. The oxide had only insignificant influence on magnetism of particles.

Small FeCo $(\approx 85 \%)$ particles showed a mixture of bcc and fcc phases in different proportions. Unlike the bulk FeCo alloys Mössbauer spectra of which showed only a sextet with rather narrow lines for any composition, the spectra of FeCo (85\%) particles (Figure 18(b)) had a more complicated shape. It contained a sextet relative to an FM bcc phase and a broad central peak, which could be decomposed into a doublet caused by amorphous SP Fe oxide and a small singlet. The sextet could be decomposed into two components with the hyperfine fields at $\mathrm{Fe}$ nuclei equal $H_{1}=36.3 \mathrm{~T}$ and $H_{2}=33.6 \mathrm{~T}$. A component with the larger relative area of spectrum and the higher field $H_{1}$ should be attributed to the fcc phase because its content prevailed in particles, whereas a fraction of bcc phase turned out to be insignificant. The component corresponding to the field $\mathrm{H}_{2}$ was related probably both to the bcc and fcc phases.

In spectra of the $\mathrm{FeCo}(90 \%)$ and $\mathrm{FeCo}(95 \%)$ particles a sextet was referred to the fcc phase, an oxide doublet disappeared, and a central peak could be decomposed into two singlets, one of which was attributed to a PM fcc phase, whereas another slightly shifted singlet was likely caused by small clusters of pure iron (Figure 18(c)). The Mössbauer spectrum of $\mathrm{FeCo}(83 \%)$ particles previously heated in vacuum and experienced the bcc $\rightarrow$ fcc phase transformation is shown in Figure 18(d). It demonstrates two well-resolved sextets with hyperfine fields $\mathrm{H}_{2} \approx 46.3 \mathrm{~T}$ and $H_{3} \approx 49.4 \mathrm{~T}$ in addition to a sextet with $H_{1} \approx$ $32.8 \mathrm{~T}$ related to the fcc phase of the alloy. Although the simultaneous presence of two components with fields of $\mathrm{H}_{2}$ and $\mathrm{H}_{3}$ as well as their magnitudes is characteristic of both spinels $\mathrm{Fe}_{3} \mathrm{O}_{4}$ and $\mathrm{CoFe}_{2} \mathrm{O}_{4}$, nevertheless, an area relation of the two sextets is closer to that for the inverse spinel of $\mathrm{Co}_{0.54} \mathrm{Fe}_{3-0,54} \mathrm{O}_{4}$ composition [72]. Thus, in the Co-rich alloy particles both HS and LS states of the fcc lattice, as in the case of Fe-rich FeNi, FeMn, and FePt particles, are observed.

Comparison of the concentration dependence of hyperfine field $H_{i}$ for FeCo nanoparticles with that for massive alloys is given in Figure 19. Moreover, only the most representative data are shown in order not to obscure the figure [71]. It is seen that there is a considerable diversity in the data of different authors, but the experimental points for aerosol particles do not go beyond the scatter. One reason for the discrepancies in the results of different authors might be an uncontrollable ordering of FeCo alloys. For example, it has been shown in $[74,75]$ that the hyperfine field in ordered alloys with 40-60 at.\% Co is much smaller than in disordered alloys (see Figure 19). The data [73] just hit the curve for the ordered alloys, although this issue was not touched in the work.

The initial growth of $H_{i}$ to the maximum achieved at about 30 at.\% Co content can be explained both by the dominant contribution of the polarization mechanism, 


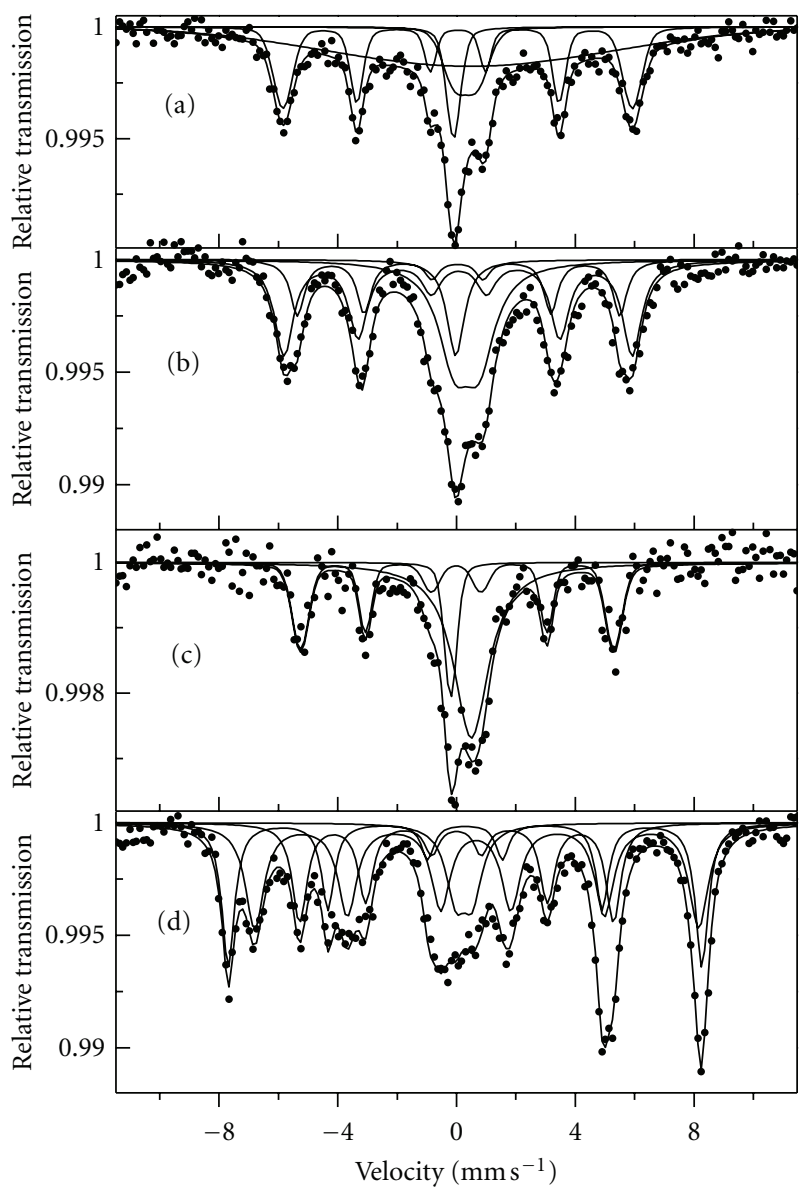

Figure 18: Concentration-dependent Mössbauer spectra of FeCo nanoparticles with a size of $D=10-15 \mathrm{~nm}$. (a) FeCo (35\%); sextet, $S_{1}=31 \%, H_{1}=36.5 \mathrm{~T}$; "sagged" line, $S_{2}=48 \%$; oxide doublet, $S_{2}=$ $13 \%$; singlet of PM phase, $S_{4}=8 \%$; (b) FeCo (85\%); two sextets: (I) $S_{1}=41 \%, H_{1}=36.3 \mathrm{~T}$ (II), $S_{2}=19 \%, H_{2}=33.6 \mathrm{~T}$; oxide doublet, $S_{3}$ $=31 \%$; singlet, $S_{4}=9 \%$; (c) FeCo (90\%); sextet $S_{1}=36 \%, H_{1}=$ $32.7 \mathrm{~T}$; a narrow singlet, $S_{2}=11 \%$, a broad singlet, $S_{3}=53 \%$; (d) FeCo $(85 \%)$ after heating in vacuum at $600^{\circ} \mathrm{C}$ for $30 \mathrm{~min}$. Three sextets: (I) $S_{1}=23 \%, H_{1}=32.8 \mathrm{~T}$; (II) $S_{2}=26 \%, H_{2}=46.3 \mathrm{~T}$; (III) $S_{3}=41 \%, H_{2}=49.4 \mathrm{~T}$; doublet, $S_{4}=10 \%$.

which increases the hyperfine field at iron nuclei, and by the growth of the magnetization of the very alloy. The influence of HS state inheritance of fcc Fe lattice cannot be also excluded which increases the lattice constant of alloy and its magnetic moment. With further increasing Co content the other competitive mechanisms become dominant which reduce $H_{i}$ or decrease the magnetization of the alloy. All these mechanisms evolve in the bcc phase in the Co concentration range of 0 to 73 at.\%. Then the alloys acquire the $\mathrm{fcc}$ structure keeping up to an $\mathrm{Fe}_{8} \mathrm{Co}_{92}$ composition. Naturally, one can expect a change of interactions as a result of the $\mathrm{bcc} \rightarrow \mathrm{fcc}$ transition. Indeed, when the content of $\mathrm{Co}$ is over 80 at.\% in alloy sample [73] and ball milling powders [77], the hyperfine field $H_{i}$ ceases to depend on the alloy composition within the diversity of the experimental data and becomes equal $H_{i} \approx 33 \mathrm{~T}$ as in pure iron. The curve of the concentration dependence of $H_{i}$ for aerosol particles

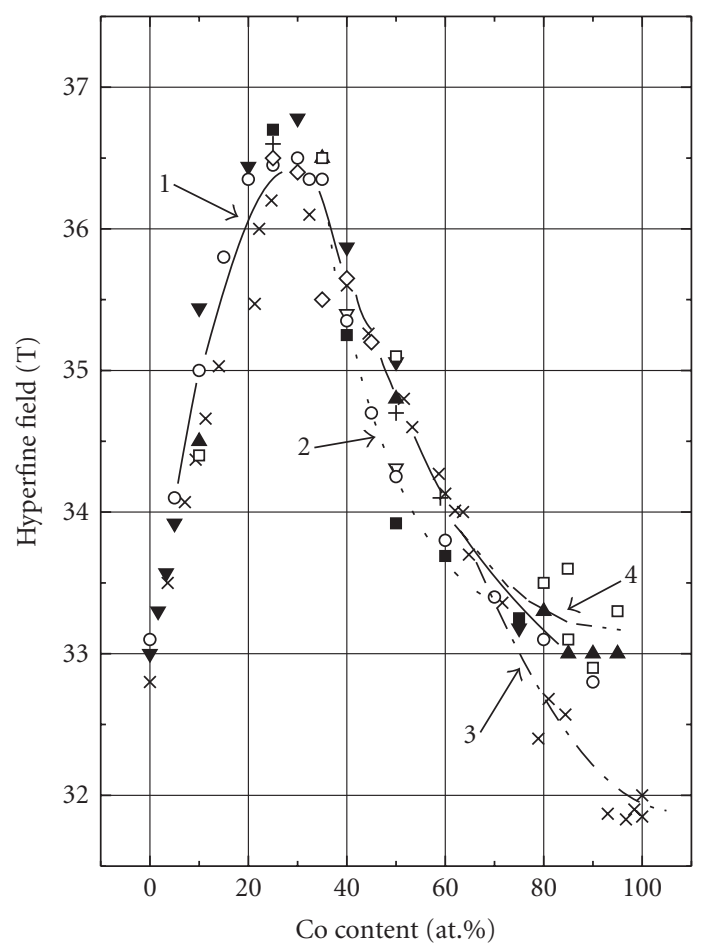

FIGURE 19: Concentration-dependent hyperfine field at the iron nuclei in FeCo alloys. Alloying components: $\mathrm{O}$ according to [73]; + for the disordered and $\boldsymbol{\square}$ for the ordered alloys [74]; $\boldsymbol{\nabla}$ for the disordered and $\nabla$ for the ordered alloys [75]; $\times$, electrolytic deposition [76]; $\boldsymbol{\Delta}$, ball milling [77]; $\diamond$, precipitation of small particles in the $\mathrm{Cu}$ matrix [78]; $\square$, nanoparticles with a size of $D$ $=10-15 \mathrm{~nm}$ [71]. The curves correspond to disordered alloy (1), ordered alloy (2), electrolytically deposited films (3), nanoparticles (4).

in the range of cobalt-rich alloys also tends to the value $H_{i} \approx 33.1 \mathrm{~T}$. This is probably due to release of small iron clusters in the system. Meanwhile, in contrast to the results for samples prepared by alloying or ball milling components, significantly different concentration dependence of $H_{i}$ was obtained for the electrodeposited Co-rich films (Figure 19) [76]. One tried to explain this difference by the presence of various lattice defects [77], but its reason can be directly linked to the influence of the substrate, resulting in a strong deviation of the intensities of the 2 nd and 5th peaks in the sextet [76] from the ratio $3: 2: 1$ specific to a random orientation of magnetic moments. Owing to this in (6597 at.\%) Co concentration range, where the fcc structure of the films holds, the resulting field $H_{i}$ is lower than that at alloyed samples and aerosol particles. When the Co concentration in the alloy is higher than 97 at.\%, the electrodeposited films have a hcp structure, and $H_{i}$ gets a value of about $31.9 \mathrm{~T}[76]$.

The curve shape in Figure 19 roughly appears similar to that for massive alloys, and nanoparticles. This denotes that the origin of concentration dependence for FeCo alloy which remained unclear so far, is at atomic level. Correlation between the concentration dependences of hyperfine field at 


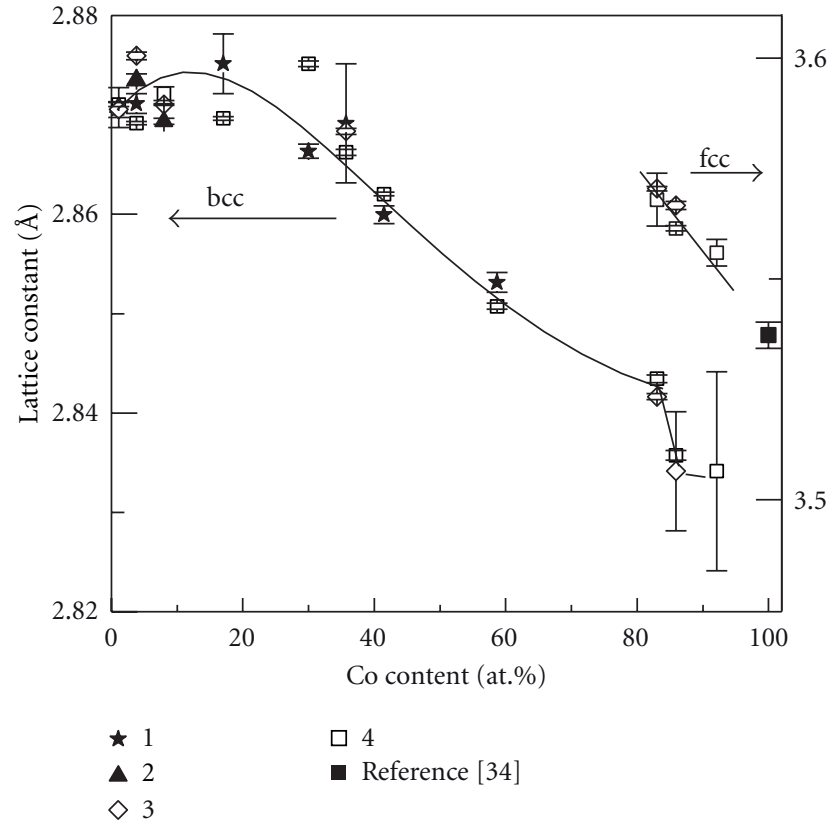

FIGURE 20: Concentration dependence of the lattice parameter $a$ of the $\mathrm{Fe}_{100-x} \mathrm{Co}_{x}$ particles produced at an argon pressure of (1) 67, (2) 133, (3) 400, and (4) $1330 \mathrm{~Pa}$.

Fe nuclei and lattice constant at FeCo nanoparticles has been displayed in [79].

As seen in Figure 20, at increasing Co content in $\mathrm{Fe}_{100-x} \mathrm{Co}_{x}$ nanoparticles the lattice constant curve is similar to the curve in Figure 19. At first, the bcc lattice constant somewhat increases, attaining the maximum value $2.875 \AA^{\prime}$ nearby $x=20$ and then smoothly decreases to value $2.841 \AA^{\prime}$ at $x \approx 82$ where bcc $\rightarrow$ fcc transformation takes place. This transformation is accompanied with a sharp reduction of the bcc lattice constant and occurrence of an fcc phase with the lattice constant equal approximately $3.554 \AA^{\prime}$. Note that in the point of phase transition the volumes per atom are almost equal $\left(11.47 \AA^{3}\right.$ for the bcc phase and $11.40 \AA^{3}$ for the fcc one). In the range $x \approx 83-92$ the bcc and fcc phases coexist. At $x \rightarrow 100$ the fcc lattice constant tends to the value for aerosol Co particles $\left(3.5373 \pm 0.003 \AA^{\prime}[80]\right)$.

The revealed correlation between concentration dependences of hyperfine field at Fe nuclei and those for lattice constants of nanoparticles has a quantum mechanical explanation. According to Slater [81] the isolated atoms with a partially filled shell and also a crystal of such atoms can lower its energy due to establishing the identical direction of electron spins (spin polarization) that gives rise to magnetization of a crystal. However, owing to a finite width of energy bands, the energy of crystal increases, as soon as it becomes magnetized. If the partially filled bands are too wide, the mechanism increasing the crystal energy can prevail over exchange effects which tend to provide the spontaneous magnetization lowering its energy. Then the crystal will appear nonmagnetic. As the band width depends on a distance between atoms, the magnetic state of crystal is defined by the change of lattice constant. Approximate calculations for iron have revealed that the interatomic spacing, at which magnetism disappears, appears less than the equilibrium distance between atoms. Therefore, ferromagnetism of iron should hold even at some reduction of the bcc lattice constant (exact theoretical estimations are absent).

As Slater noted, the energy distinction between ferromagnetic and antiferromagnetic states of crystal is extremely little which strongly complicates their theoretical identification. The existing theories consider magnetic interaction of the next atoms by means of Heisenberg exchange integral $J$ [82]. Depending on reduction of the ratio of the internuclear distance to a radius of the unfilled shell, a positive magnitude $J$ at first smoothly grows to a maximum describing a ferromagnetism region, then it rather sharply falls and changes the sign. The region of negative values $J$ is connected with antiferromagnetic state of substance.

The average hyperfine field of $\mathrm{Fe}_{100-x} \mathrm{Co}_{x}$ nanoparticles shows similar behaviour in a ferromagnetic region. The ferromagnetism of nanoparticles slightly grows to a maximum at the small additive of cobalt increasing the bcc lattice constant. The subsequent increase of the Co content leads to reduction of the lattice constant and particle ferromagnetism. Thus, it is available the full symbasis of considered parameters. The latter proves to be true also by that the transition from a maximum of curves in Figures 19 and 20 to a point of bcc $\rightarrow$ fcc transformation is accompanied with a close reduction of both the magnetization (an average hyperfine field) of particles in 36.6/33 = 1.11 times, and the lattice constant in 2.875/2.841 $=1.012$ times.

Mössbauer spectra of FeCo particles of all compositions before and after bcc $\rightarrow$ fcc transition (Figure 18) consist of a sextet of lines, which defines an average hyperfine field, and also from a wide central paramagnetic peak. The part of this peak including the contribution from Fe oxide, obviously, should decrease with growth of the Co additive. On the other hand, the relative fraction of a paramagnetic singlet considerably increases at $x>80$ at.\%. This can be connected with superparamagnetism of the smallest groupings of iron arising in the fcc Co lattice and somewhat expanding it near a bcc $\rightarrow$ fcc transition of FeCo alloy (Figure 20). Larger atomic Fe groupings give a ferromagnetic sextet of lines with almost the same average hyperfine field as that at a massive iron. And though these groupings are not found out by X-ray, their lattice constant, obviously, should not differ considerably from that for a massive iron. In other words, in a point of bcc $\rightarrow$ fcc transition there is its partial stratification on small particles of pure iron and the particles of cobalt containing an impurity of smaller iron aggregations. Such immiscibility, obviously, arises already at early stages of process of formation of aerosol particles of the given composition.

4.4.6. $\mathrm{FeCr}$. The structure and phase composition of $\mathrm{FeCr}$ alloys above $300^{\circ} \mathrm{C}$ are defined by the equilibrium constitution diagram (Figure 21) [68]. At lower temperatures, 


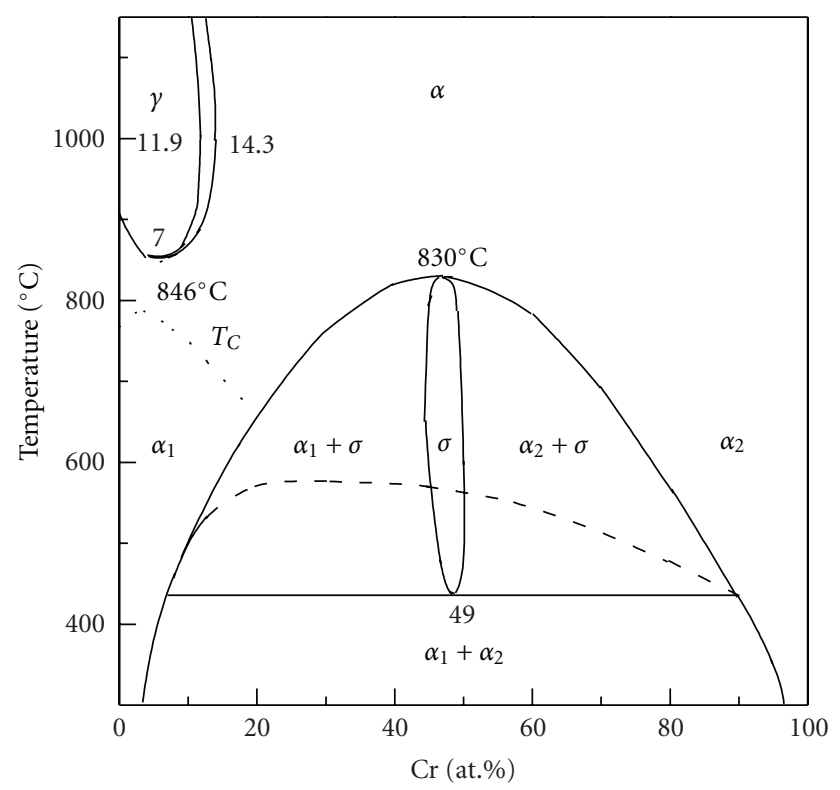

FIgure 21: Constitution diagram for FeCr system (see [68]).

thermal equilibrium cannot be established because of slow diffusion of atoms, and alloys can stay in a metastable state for a long time. A different situation occurs in nanoparticles within which, due to their small size, mixing of atoms proceeds with much higher rate than in the bulk substance, and the thermal equilibrium is established even at temperatures below $300^{\circ} \mathrm{C}$ [83].

The structure and Mössbauer spectra of metastable $\mathrm{Fe}_{100-x} \mathrm{Co}_{x}(x=2.36 \div 80.3)$ bulk alloy and nanoparticles with an average size of $13-15 \mathrm{~nm}$ produced by fast evaporation of the predegassed in vacuum initial alloys in an Ar atmosphere have been studied [83-85]. X-ray patterns for the bulk alloys show only a bcc phase without any foreign lines. The lattice constant of the bcc phase slightly rises with $\mathrm{Cr}$. The bcc lines are observed also in X-ray patterns of the nanoparticles. However, as $\mathrm{Cr}$ content in the nanoparticles exceeds a value of 23.4 at.\%, an appearance, followed by intensification, and, finally, an attenuation of lines referred to the $\sigma$-phase are observed. In $\mathrm{FeCr}$ (47.68 at.\%) nanoparticles the $\sigma$-phase dominates over the bcc phase. Traces of $\sigma$-phase are kept even at $\mathrm{FeCr}$ (83.03 at.\%) nanoparticles. The lattice constant of $\sigma$-phase $(\mathrm{a}=8.78 \AA, c=4.55 \AA$ [83]) agrees well with the data of Bergman and Shoemaker [86] for $\mathrm{FeCr}$ (46.5 at.\%) alloy annealed at $650^{\circ} \mathrm{C}(a=8.7995 \AA, c=$ $4.5442 \AA$ ) and with the data of Dickins et al. [87] for FeCr (50 at.\%) ( $a=8.790 \AA, c=4.559 \AA)$. The results of studying structure and composition of FeCr particles of 13-18 nm size with a different $\mathrm{Cr}$ content are given in works $[84,85]$.

Concentration dependence of the bcc lattice constant in $\mathrm{FeCr}$ alloy at room temperature is shown in Figure 22 [83]. At the low $\mathrm{Cr}$ content (2.36-8.86 at.\%) the lattice constant for nanoparticles is found to be larger than that for a bulk alloy, though the values of hyperfine fields at Fe nuclei do not differ. In contrast to pure Fe aerosol particles whose lattice constant is identical to that of the bulk substance, $\mathrm{FeCr}$ alloys with the

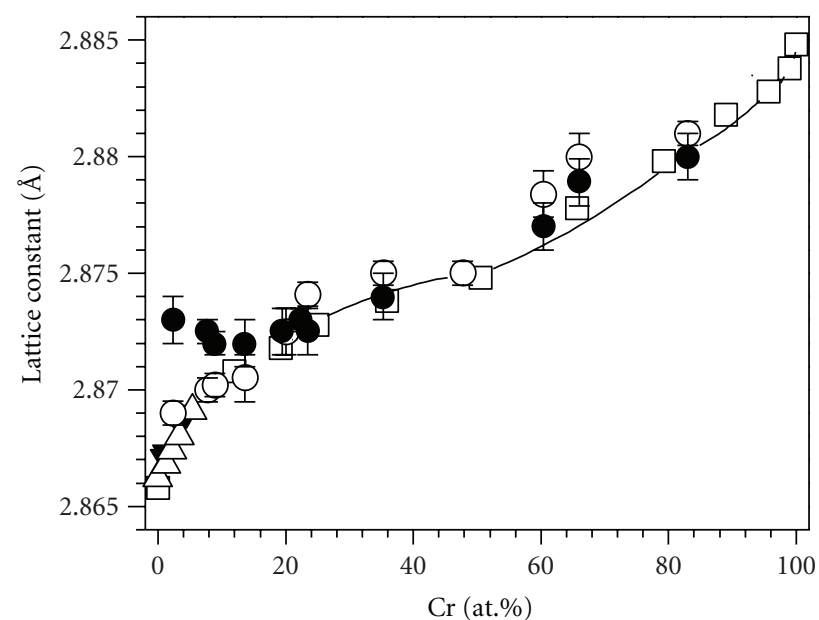

Figure 22: Concentration dependence of the bcc lattice constant for FeCr alloy at room temperature. Metastable bulk alloy: $\square[88,89]$; $\boldsymbol{\nabla}[90] ; \triangle[91] ; \mathrm{O}[83,84]$; nanoparticles with a size of $13-15 \mathrm{~nm}$ $[83,84]$.

low Cr impurity (2.36-8.86 at.\%) have an appreciably larger lattice constant compared to that of nanoparticles.

As can be seen in Figure 22, at $\mathrm{Cr}$ content $>16$ at.\% the data for nanoparticles satisfactorily agree with the data of other authors obtained for metastable homogeneous bcc alloys. A sharp bend in the smoothed visual curve is well observed near the equiatomic alloy composition, which indicates a trend to $\alpha \rightarrow \sigma$ phase transition. Just at this $\mathrm{Cr}$ concentration the bcc lines of the X-ray patterns for the nanoparticles are attenuated at the expense of the intensification of $\sigma$-phase lines.

Mössbauer spectra of bulk alloy with different $\mathrm{Cr}$ content are shown in Figures 23 and 24. Similar Mössbauer spectra of $\mathrm{FeCr}$ nanoparticles in size of about $14 \mathrm{~nm}$ are given in Figure 25 and Figure 26. The corresponding distributions of hyperfine fields at Fe nuclei are shown in the inserts of the figures. At the lowest $\mathrm{Cr}$ content (2.36 at.\%) the spectra of the bulk alloy and nanoparticles contain sextets of rather narrow lines, which practically do not differ from that for pure Fe. The spectrum of nanoparticles exhibits an additional SP (superparamagnetic) split doublet which belongs to Fe oxides, and the very spectrum is "sagged" at the central part because of the hyperfine field distribution. After heating of the powder in vacuum at $300^{\circ} \mathrm{C}$ for $1 \mathrm{~h}$ or at $500^{\circ} \mathrm{C}$ for $0.5 \mathrm{~h}$ the sagging of spectrum completely disappears. The annealing of the powder at $500^{\circ} \mathrm{C}$ gives rise to appearance of structural lines related to $\mathrm{Fe}_{3} \mathrm{O}_{4}$ and the additional sextets with hyperfine fields of $44.25 \mathrm{~T}$ (a fraction of total spectrum area $S=28.4 \%$ ) and $47.8 \mathrm{~T}$ $(S=7.4 \%)$. This is caused by the Fe nuclei occupying octahedral and tetrahedral sites in the lattice of spinel, respectively. A relative area of the SP doublet is somewhat increased (from $20 \%$ up to $31.5 \%$ ). It can be explained by supposition that at high-temperature heating the outward layer of the primary amorphous shell is crystallized in spinel, whereas the metallic core continues to slowly oxidize even in vacuum of $2.7 \cdot 10^{-3} \mathrm{~Pa}$. A newly forming oxide appears 


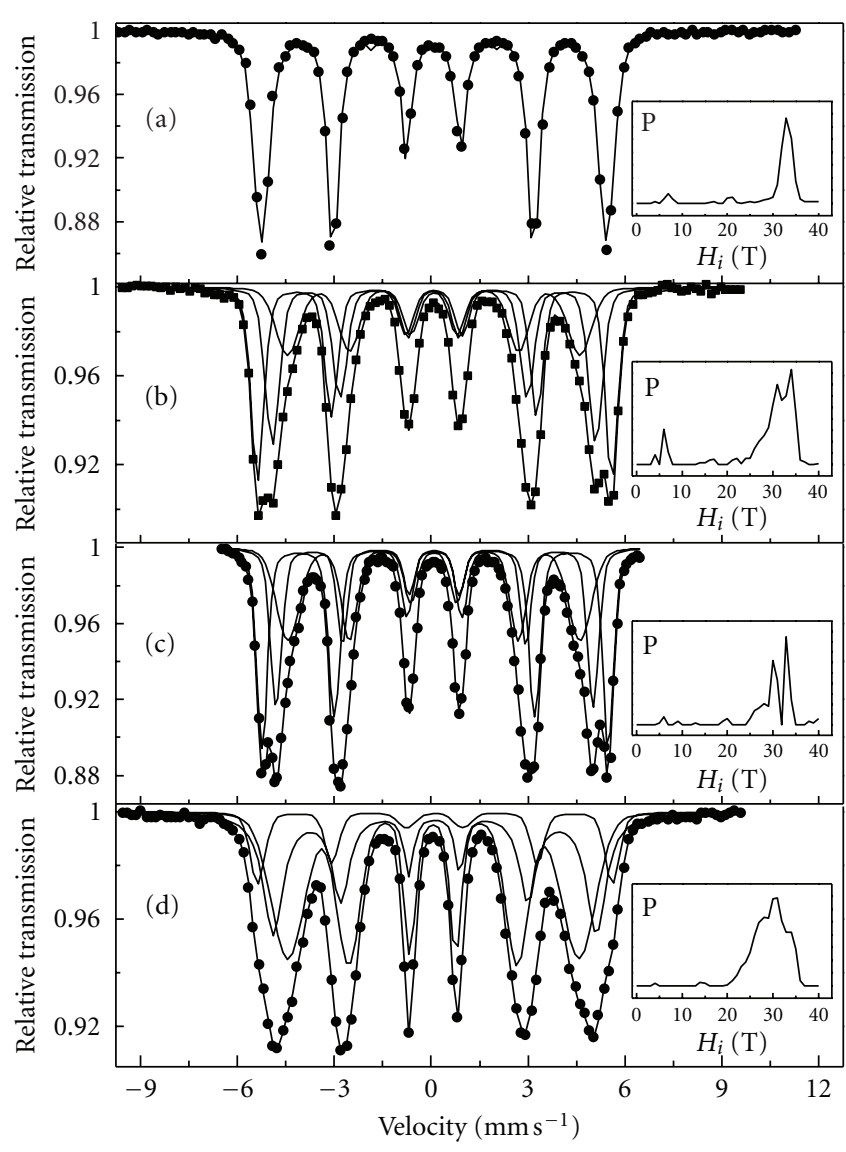

Figure 23: Mössbauer spectra of bulk $\mathrm{FeCr}$ alloy with low $\mathrm{Cr}$ content. (a) 2.36 at.\%; (b) 7.69 at.\%; (c) 8.86 at.\%; (d) 13.62 at.\%. Hyperfine field distributions are shown in the inserts.

to be amorphous and superparamagnetic owing to the low thickness. It is known $[18,20]$ that the SP doublet is lacking in the spectra of pure Fe nanoparticles though an amorphous oxide is likely to constitute no less than $30 \%$ of the particle volume. However, the additive of some metals to iron leads to occurrence of oxide doublet in Mössbauer spectra. This, apparently, is connected with some decrease of mean-square deviations of $\mathrm{Fe}$ atoms in an amorphous $\mathrm{Fe}$ oxide shell of particles. The latter, in turn, raises Mössbauer effect probability and consequently superparamagnetism of Fe oxide becomes appreciable. Even a little $\mathrm{Cr}$ addition to $\mathrm{Fe}$, evidently, disturbs a cubic symmetry that is manifested essentially strongly at the particle surface. A rise of the electric field gradients and their interaction with quadrupole moment of $\mathrm{Fe}$ nucleus results in quadrupole splitting in the Mössbauer spectra leading to the oxide split doublet. At the same time the electric field gradients owing to $\mathrm{Cr}$ impurity have no substantial effect on the sextet shape in the spectrum of $\mathrm{FeCr}$ (2.36 at.\%) nanoparticles. The SP oxide doublet is present in the spectra of $\mathrm{FeCr}$ nanoparticles of any composition. Based on its relative areas, the oxide content is from $20 \%$ to $40 \%$.

The Mössbauer spectra for the bulk FeCr ( $\geq 7.69$ at.\%) alloys appreciably change. A sextet is clearly decomposed

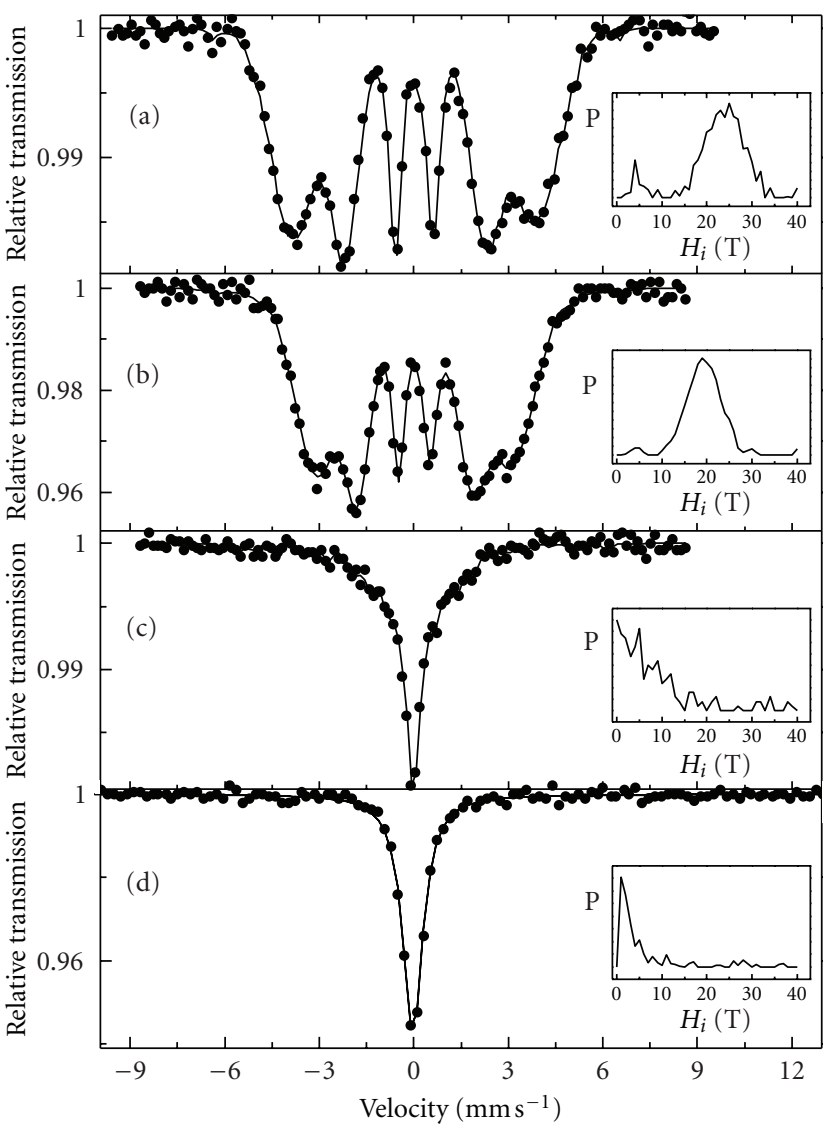

FIgURE 24: Mössbauer spectra of bulk FeCr alloy with high $\mathrm{Cr}$ content. (a) 23.4 at.\%; (b) 47.68 at.\%; (c) 66,02 at.\%; (d) 83.03 at.\%. Hyperfine field distributions are shown in the inserts.

into three components, the hyperfine field of one of which is close to that for pure $\mathrm{Fe}\left(H_{0} \approx 33 \mathrm{~T}\right)$ and two others are lower $\left(H_{1} \approx 30.5 \mathrm{~T}, H_{2}=2.80 \mathrm{~T}\right)$ (Figure 23). Annealing of $\mathrm{FeCr}\left(8.86\right.$ at.\%) alloy at $300^{\circ} \mathrm{C}$ in vacuum $2.7 \times 10^{-3} \mathrm{~Pa}$ for $2 \mathrm{~h}$ practically has no any effect on both the lattice constant of the bulk bcc lattice and the Mössbauer spectrum. The Mössbauer spectrum of the $\mathrm{FeCr}$ (47.68 at.\%) alloy, which contains two narrow central lines and two broaden outer peaks at each side, corresponds to a wide hyperfine field distribution centered at about $19 \mathrm{~T}$ (Figure 24(b)). The Mössbauer spectrum of the $\mathrm{FeCr}$ (66.02 at.\%) alloy holds a broadened central peak with vague sextet features (Figure 24(c)). Finally, the spectrum of $\mathrm{FeCr}$ (83.03 at.\%) alloy shows only a singlet for which a hyperfine field distribution is grouped nearby zero value with a full width at half maximum of $\approx 3 \mathrm{~T}$ (Figure $24(\mathrm{~d})$ ).

Comparison of Figures 25 and 26 with Figures 23 and 24 shows that the Mössbauer spectra for bulk alloys and nanoparticles significantly differ. In the case of $\mathrm{FeCr}$ (7.69 at.\%) and $\mathrm{FeCr}$ (8.86 at.\%) nanoparticles, the sextet lines are broadened and the oxide split doublet is present. The sextet can be decomposed, at least, into three components with hyperfine fields $H_{i}=27.1,29.19,32.2 \mathrm{~T}$ for $\mathrm{FeCr}$ (7.69 at.\%) and into two components with hyperfine fields $H_{i}=26.77,33.22 \mathrm{~T}$ for $\mathrm{FeCr}$ (8.86 at.\%). A shape of the 


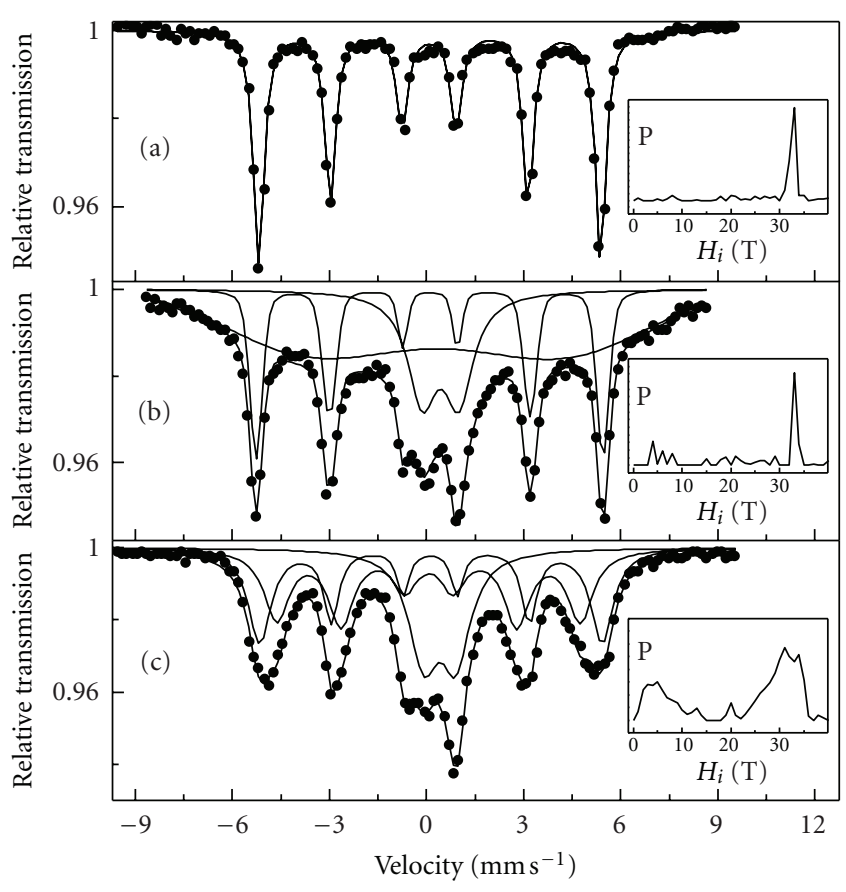

Figure 25: Mössbauer spectra of FeCr nanoparticles of $D=14 \mathrm{~nm}$ size with low $\mathrm{Cr}$ content. (a) Pure iron; (b) 2.36 at.\%; (c) 7.69 at.\%. Hyperfine field distributions are shown in the inserts.

sextet lines for $\mathrm{FeCr}$ (13.62 at.\%) nanoparticles is somewhat different, and the sextet can be decomposed not only into two $\left(H_{i}=29.65,32.84 \mathrm{~T}\right)$ but also even to three components. For FeCr (23.4 at.\%) a difference in the spectra of bulk and nanoparticles becomes essential since a singlet shows up in the nanoparticle spectrum. With the further $\mathrm{Cr}$ increase, the singlet becomes dominant.

A dependence of the averaged hyperfine fields on concentration for $\mathrm{FeCr}$ alloys is shown in Figure 27 in comparison with the data of other authors. A value of the hyperfine field for $\mathrm{FeCr}$ (20 at.\%) nanoparticles with a size of $21 \mathrm{~nm}$ at $4 \mathrm{~K}$ is also given in Figure 27. At $\mathrm{Cr}$ content $\leq 24$ at. $\%$ the data for the bulk alloy and nanoparticles do not deviate from the average values obtained by other authors. The further sharp falldown of the curve with $\mathrm{Cr}$ is accompanied by attenuation of ferromagnetism transforming into paramagnetism. Nanoparticles were found to become predominantly PM at less Cr content $(\approx 35$ at. $\%)$ than the bulk alloy $(\approx 68$ at. $\%)$. However cooling to $4 \mathrm{~K}$ (curve 3 ) restores the ferromagnetism of alloys. The Cr-rich alloys are purely PM at room temperature (curve 5) and $\mathrm{AF}$ at $4 \mathrm{~K}$ (curve 4). As for $\sigma$-phase it is certainly paramagnetic above Curie point $T_{C}=160 \mathrm{~K}$ [95]. Occurrence of singlet in Mössbauer spectra of nanoparticles (Figure 26(a)) can be attributed to an appearance of $\sigma$-phase. The further intensification of singlet (Figures 26(b) and 26(c)) is caused with summation of contributions of PM $\sigma$-phase and Cr-rich bcc phase. The Curie temperature $T_{C}$ in the metastable quenched $\mathrm{FeCr}$ alloys decreases to $80-50 \mathrm{~K}$ in the $80.0-81.8$ at. $\% \mathrm{Cr}$ range [99]. The $\mathrm{FeCr}(>80$ at.\%) alloys and pure $\mathrm{Cr}$ are antiferromagnetic.

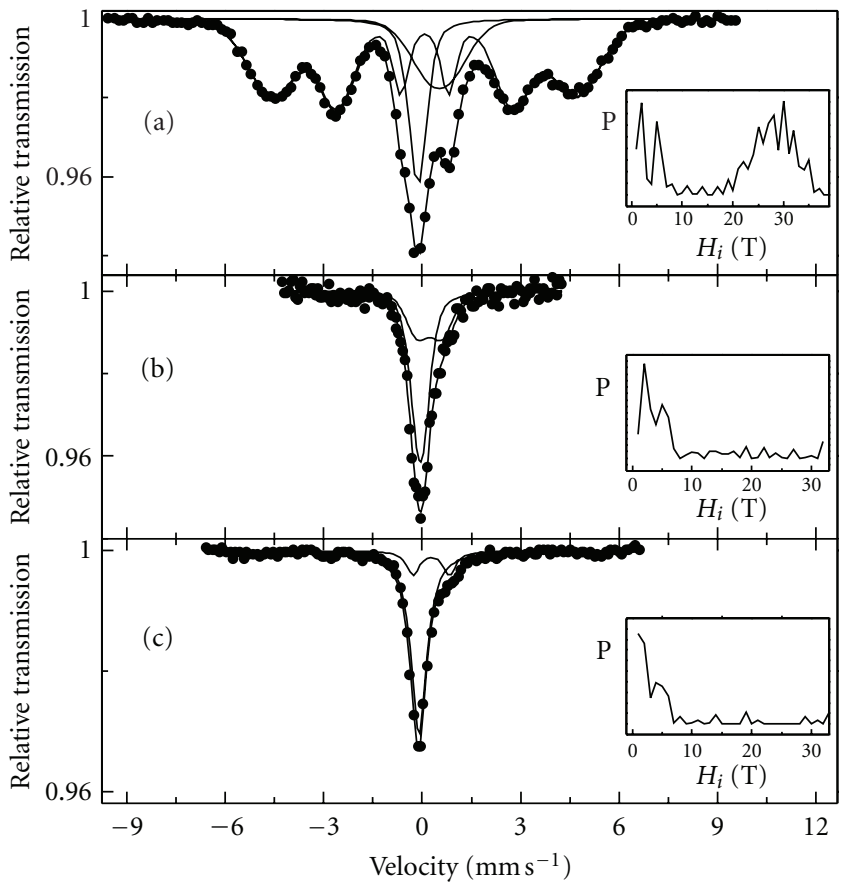

FIGURE 26: Mössbauer spectra of FeCr nanoparticles with high $\mathrm{Cr}$ content. (a) 23.4 at.\%; (b) 47.68 at.\%; (c) 83.03 at.\%. Hyperfine field distribution are shown in the inserts.

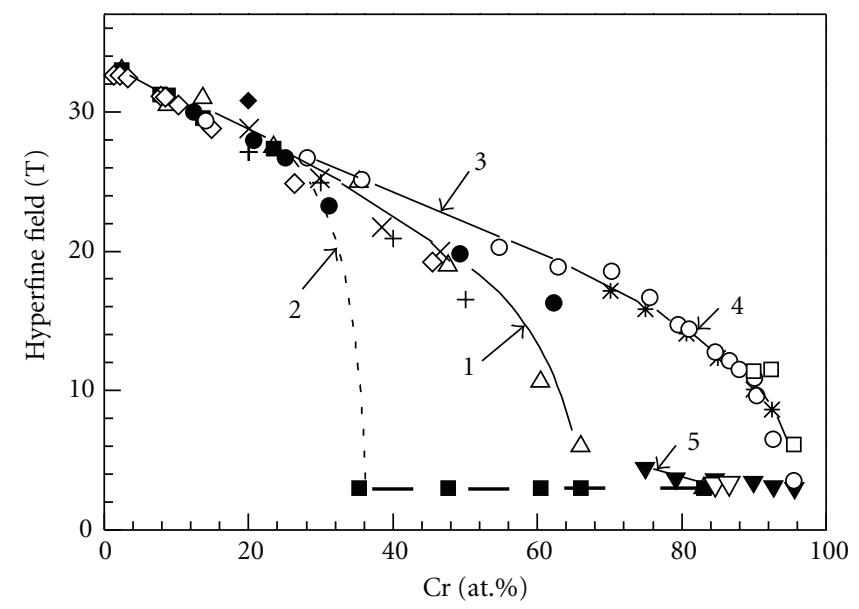

FIGURE 27: Concentration-dependent hyperfine field at Fe nuclei in FeCr alloy. Quenched bulk alloy: $\triangle[84] ;+[92] ; \bullet[73] ; \times[93] ; *$ (5 K) [94]; $\nabla$ [95]; $\diamond ~[96] ; \nabla(40 \mathrm{~K})$ [97]; O (4.2 K) [97]; $\square$ (1.7 K) [97]. Nanoparticles: $\sim 14 \mathrm{~nm}$ particles [84]; $21 \mathrm{~nm}$ particles at $T=4.2 \mathrm{~K}[98]$; visual curves: 1 , bulk alloy $(300 \mathrm{~K}) ; 2$, nanoparticles $(300 \mathrm{~K}) ; 3$, bulk alloy $(4.2 \mathrm{~K})$; 4 , averaged data for the bulk alloy at 4.2 and $1.7 \mathrm{~K}$; and 5, averaged data for the bulk alloy at 300 and $40 \mathrm{~K}$.

Comparison of magnetic properties of bulk $\mathrm{FeCr}$ alloys and nanoparticles produced from them has been fulfilled in [85]. The hysteresis loops for the different $\mathrm{FeCr}$ alloys and nanoparticles taken at room temperature are shown in Figure 28. For bulk alloys the loops are typical; namely, they are a soft magnetic material with a negligible coercivity $\left(H_{C}\right)$ which does not exceed 15 Oe in any composition. Let 


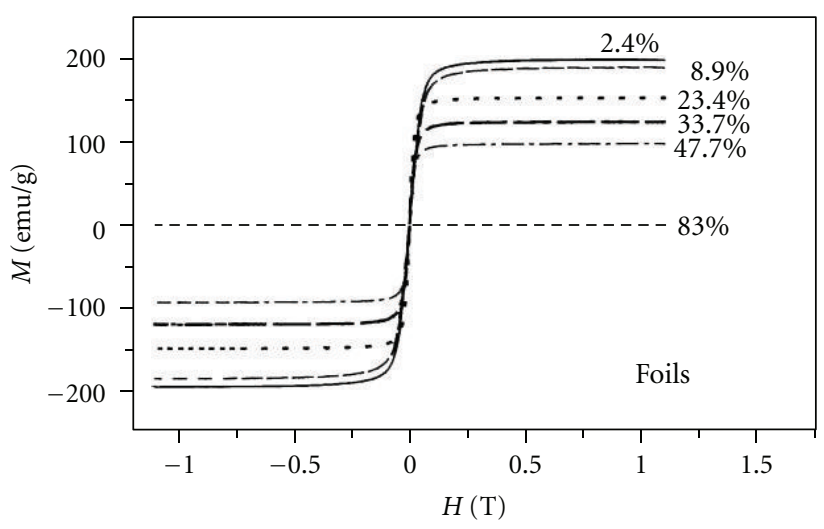

(a)

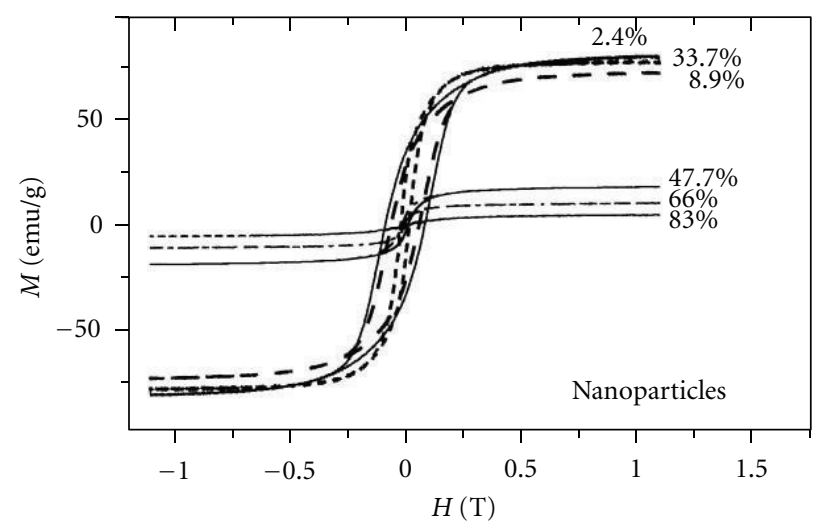

(b)

FIGURE 28: Magnetization curves at room temperature for different $\mathrm{Fe}_{100-x} \mathrm{Cr}_{x}$ alloys (a) and nanoparticles of $D=13-15 \mathrm{~nm}$ size (b). The values of $x$ are given for every curve.

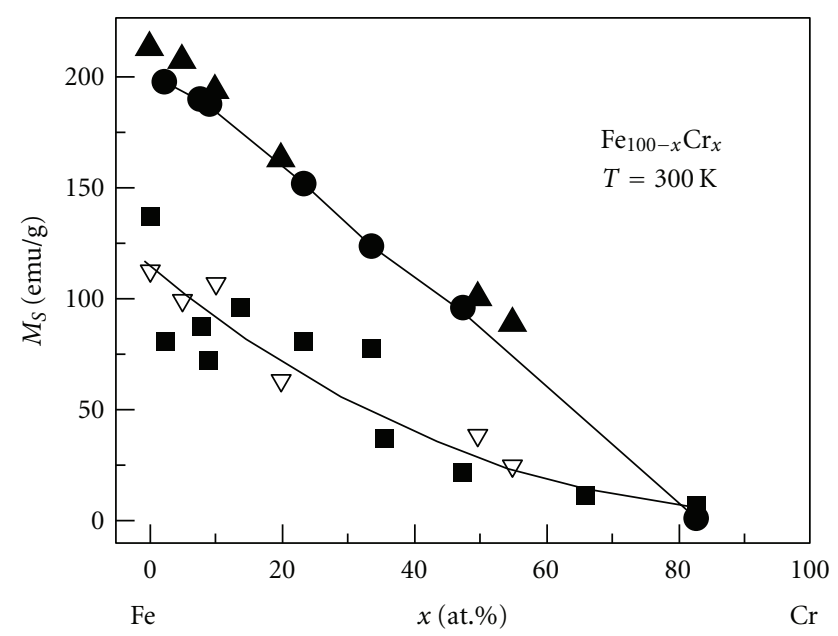

Figure 29: Concentration dependences of saturation magnetization, $M_{S}$, for both bulk FeCr alloys and nanoparticles defined at the applied field $H=1.1 \mathrm{~T}$ and at room temperature. Bulk alloy: - [85], $\boldsymbol{\Lambda}$ [100]. Aerosol particles: $\boldsymbol{\square}$ [85], $\nabla 21 \mathrm{~nm} \mathrm{[100].}$

us draw the reader's attention to the fact that hysteresis loops are rectangular in case of bulk alloys whereas at $\mathrm{FeCr}$ nanoparticles they are inclined similarly to that for iron nanoparticles. The inclination of hysteresis loops, obviously, is caused by presence in the sample of small chains of the particles connected by magnetic forces.

Hysteresis loops allow one to define saturation magnetization $\left(M_{S}\right)$ (Figure 29) and coercivity $H_{C}$ (Figure 30). A magnitude $M_{S}$ at a bulk alloy is approximately in 2 times above that at nanoparticles with a low $\mathrm{Cr}$ content, but as the latter grows, the magnitudes of $M_{S}$ approach, becoming equal in an alloy with composition near $\mathrm{Fe}_{20} \mathrm{Cr}_{80}$. On the contrary, the value $H_{C}$ at particles close to that for $\mathrm{Fe}_{20} \mathrm{Cr}_{80}$ foil sharply increases when diminishing $\mathrm{Cr}$ content. This distinction of magnetic properties of bulk alloys and particles, obviously, is caused by difficult exchange

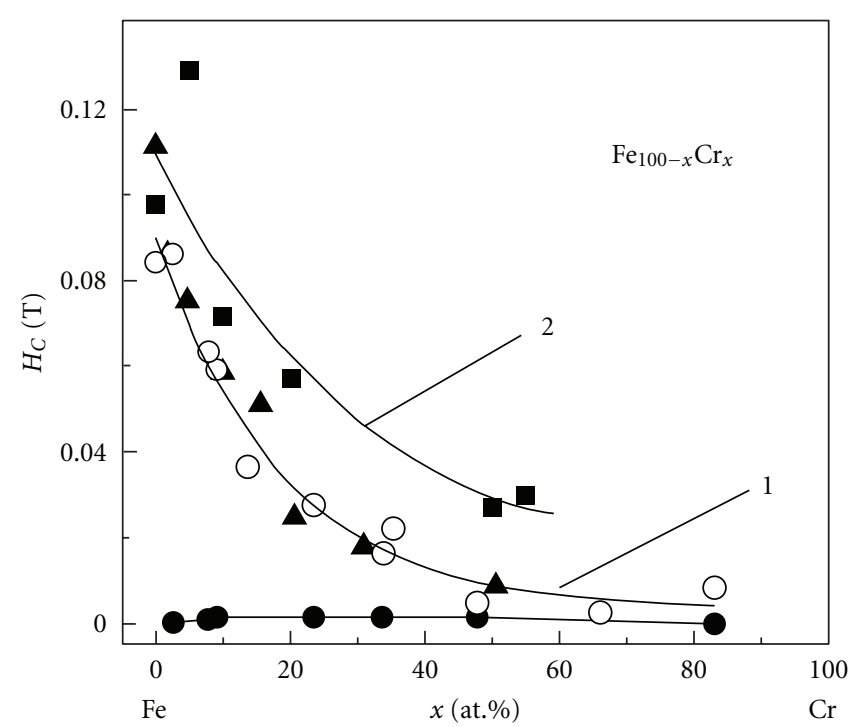

Figure 30: Concentration dependence of coercivity $H_{C}$. Bulk alloy: - [85]. Aerosol particles: O [85], $\square 21 \mathrm{~nm} \mathrm{[100],} \boldsymbol{\Delta} 15-20 \mathrm{~nm}$ [101]. 1,2 lines to guide the eye.

interaction of core with Fe oxide shell and presence of $\sigma$ phase in particles. The metastable massive alloy has only a bcc structure and does not contain $\sigma$-phase. Influence of surface oxide at the bulk alloy or particles with the large $\mathrm{Cr}$ content on their magnetic properties appears negligible.

The questions of splitting Mössbauer spectra and magnetization of massive $\mathrm{FeCr}$ alloys have been in detail discussed in [84]. Many investigators observed a splitting of outer sextet lines into two components in the Mössbauer spectra of quenched solid Fe solutions doped by a number of elements. Flinn and Ruby [102] explained its appearance by occupying the first coordination sphere with impurity atoms in a bcc Fe lattice in accordance with the binomial distribution law. Later Wertheim et al. [103] extended the approach of Flinn and Ruby to the first two coordination spheres of 
Fe atom which have eight nearest neighbors and six nextnearest neighbors. It was assumed that in disordered iron alloys the internal hyperfine field $H_{i}$ obeys some equation parameters which are defined by a sum of contributions by every coordination sphere. One can calculate $H_{i}$ for the given values of parameters and determine an individual sextet in the Mössbauer spectrum. Using various combinations of parameters the observed spectrum can be fitted by a simulation curve. A fitting efficiency depends on the choice of the parameters. This method makes it possible to get rather a good description of complex spectra even when the binomial distribution law is not applicable, that is, when a basic supposition about the random distribution of impurity atoms is violated as in ordered or concentrated solutions.

So, the model of Wertheim et al. is valid only for dilute solute concentrations, but even in this case the model of the nearest neighbors is disputable (see, e.g., a review of Fultz [104]). In Fe-rich FeCr alloys the model of occupation of the nearest coordination spheres turns out incapable to describe satisfactorily the observed Mössbauer spectra [84]. Looking for other explanations, it is necessary to consider that a sextet splitting in the Mössbauer spectra of the quenched $\mathrm{FeCr}$ alloys may be caused with two reasons at low $\mathrm{Cr}$ content, namely, (1) the splitting can have a "memory" effect about a high-temperature fcc phase; (2) the alloy has a tendency to decompose itself into two stable phases $\alpha_{1}$ and $\alpha_{2}$. The decomposition of $\mathrm{FeCr}(7.69-13.62$ at.\%) spectra into three components (Figures 23(b), 23(c), and 23(d)) evidently can be caused by these reasons. As for the capabilities to exhibit the "memory" effect in Fe-rich alloys, it is worthy remind that the HS and LS magnetic states of the high-temperature fcc phase are kept in the resulting bcc structure of Fe-rich FeNi, FeMn, and so forth.

The mean hyperfine field (Figure 27) practically coincides with the hyperfine field value of one of the sextet components in the $\mathrm{FeCr}$ spectra relative to $H_{1}=31.0 \mathrm{~T}$ for FeCr (7.69 at.\%), $H_{1}=30.38 T$ for FeCr (8.86 at.\%), and $H_{1}=30.98 \mathrm{~T}$ for $\mathrm{FeCr}$ (13.62 at.\%). These components can be referred to bcc $\alpha$-phase of the alloy itself. At the same time, the hyperfine fields $H_{0}=33.95,33.11 \mathrm{~T}$, and $34.40 \mathrm{~T}$ of another sextet component are higher than the mean field strength, which is evidently the result of the HS state in a high-temperature fcc keeping in some domains of the bcc lattice under quenching. The third sextet component with $\mathrm{H}_{2}$ $=28.05,27.98$, and $27.91 \mathrm{~T}$ can be attributed to an admixture of $\left(\alpha_{1}+\sigma\right)$-phases or to LS state of the high-temperature fcc structure in bcc phase. In more concentrated solutions a nature of the deformation of the Mössbauer spectra is different and connected with possible decomposition of the initial alloy into two Fe-rich and Cr-rich phases.

Because of the extremely low rate of the formation of $\sigma$-phase, the Mössbauer spectra for bulk alloys annealed after quenching contain only $\alpha_{1}$ - and $\alpha_{2}$-phases in different proportions, with $\alpha_{2}$-phase displaying as a PM singlet, and $\alpha_{1}$-phase exhibiting as a FM sextet. The origin of $\sigma$-phase correlates with appearance of singlet in the Mössbauer spectra (Figure 26) and with the outset of hyperfine field decrease (Figure 27$)$ at FeCr ( 24 at.\%) nanoparticles. The further drop of the hyperfine field (Figure 27, curve 2), which is sharper than it could be expected from the constitution diagram (Figure 21), is, likely, caused by transition of the decreasing fraction of the FM bcc phase into the SP state. In FeCr ( $\geq 35$ at.\%) alloys the residual FM $\alpha_{1}$-phase only slightly widens the central peak (Figure 26(b)) whereas paramagnetic $\sigma$-and $\alpha_{2}$-phases cannot be separated from the central peak.

A tendency of the growth of $\sigma$-phase clusters in the bulk alloys is displayed by a sharp bend in the dependence for the lattice constant on concentration (Figure 22) and by a drastic drop of the hyperfine field strength at 50 at.\% Cr (Figure 27, curve 1). The falldown of the hyperfine field is not so sudden when temperature decreases to $4 \mathrm{~K}$ (Figure 27, curve 3 ). The bulk FeCr $(>70$ at.\%) alloys are PM at room temperature (Figure 27, curve 5) and become $\mathrm{AF}$ at $4 \mathrm{~K}$ (Figure 27, curve 4).

As for mechanism of decomposition of metastable alloys into two phases, according to the low-angle neutron scattering data, small chemical clusters are already present in the quenched $\mathrm{Fe}_{1-x} \mathrm{Cr}_{x}(x=0.15 ; 0.30 ; 0.50 ; 0.73)$ alloys, and a degree of clusterization increases with growth $x$ [105]. Undoubtedly, there is the equilibrium distribution of the composition fluctuations in the $\alpha$-phase of a solid homogeneous solution. At temperatures below $830^{\circ} \mathrm{C}$, the distribution is transformed in such a way that $\alpha_{1^{-}}, \alpha_{2^{-}}$, and $\sigma$-clusters are stable, and the quantitative relation is defined by the lever rule. The clusters can grow due to diffusion of atoms at favorable conditions. Hence, the decomposition of the quenched bulk alloy into two phases occurs only at cluster level. Such clusters cannot grow essentially because of the drastic slowing down of atomic diffusion rate with decrease of temperature. Since a mixing of atoms in nanoparticles is essentially faster than in a bulk substance, the phases close to the equilibrium are formed even in the course of rather rapid cooling of particles.

4.4.7. FeCu. Stable homogeneous FeCu solutions are present only as a high temperature melt. At room temperature these melts must be decomposed to pure iron and copper because of the mutual immiscibility of both components [67]. However, after high temperature quenching, an equilibrium state for the system appears not to be attained due to a very strong deceleration of diffusion processes at low temperatures and the system may be kept in a metastable state for a long time. The results of early measurements for mechanical, electrical, and magnetic properties of similar metastable alloys have been summarized in a monograph [67]. References to numerous later works are given in [106].

Nowadays metastable $\mathrm{FeCu}$ alloys are extensively produced by ball milling (BM) technique in the course of which grains of the presumably homogeneous $\mathrm{FeCu}$ solutions arise with a mean size of $10-30 \mathrm{~nm}$. Some of them have unique properties. So, $\mathrm{Cu}$-rich alloys show a giant magnetoresistance effect which can be used to develop sensors $[5,6,107]$. Another interesting result is a discovery of Invar effect at metastable $\mathrm{Fe}_{44} \mathrm{Cu}_{56}$ alloy having an fcc structure [12]. A sharp change of thermal expansion coefficient for this alloy 
close to a temperature of $350^{\circ} \mathrm{C}$ allows one to use it as a temperature switch.

Compositions specifying the boundaries of bcc and fcc phases for Fe-rich and $\mathrm{Cu}$-rich alloys, respectively, are determined insufficiently clearly and they differ from each other in various works. Estimation of atomic volume via the Wigner-Zeitz cell showed that alloying of Fe into $\mathrm{Cu}$ merely slightly changes the lattice constant of the latter and alloying of $\mathrm{Cu}$ into the bcc Fe gives rise to its noticeable expansion [108].

It is known long time ago that the Mössbauer spectra for $\mathrm{Cu}$-rich condensates having an fcc structure show a central paramagnetic peak of complicated shape (see, e.g., $[5,109,110])$. Its nature is still insufficiently clear. This central peak is usually decomposed in a singlet and a number of doublets. The singlet is referred either to the $\mathrm{Cu}$ matrix isolated single Fe atoms or to the small fcc Fe admixture. At the same time one raised doubts against appearance of the fcc Fe $[109,111]$. The doublet is ascribed to small Fe clusters in the Co matrix. It arises due to the electric field gradient on the cluster surface.

Mössbauer spectra of metastable FeCu alloys sometimes showed, in addition to the sextet specific to the bcc Fe, also sextets with a wide spread of hyperfine field values. In particular, the very fuzzy lines of sextet with the hyperfine field $H_{i}=20.6 \mathrm{~T}$ were referred to the metastable fcc FeCu alloy and the paramagnetic singlet to the fcc Fe [112]. After high-temperature annealing the singlet remained, with the amount of the fcc Fe and of the bcc Fe increasing at the expense of the decrease of the fcc $\mathrm{FeCu}$ component. At the same time the hyperfine field related to the fcc $\mathrm{FeCu}$ component went smoothly to zero at $673 \mathrm{~K}$.

The initial amount of the fcc Fe was likely very small and it could not be observed by X-ray diffraction. To find out if the fcc Fe is formed after high-temperature treatment, the BM metastable $\mathrm{Fe}_{52} \mathrm{Cu}_{48}$ solution was annealed at $823 \mathrm{~K}$ [113]. As a result, the X-ray patterns showed a presence of only fcc $\mathrm{Cu}$ and bcc Fe phases. Meanwhile, the decrease of the sample magnetization by $30 \%$ compared to that before annealing was observed at liquid helium temperature. It was accompanied with the appearance in the Mössbauer spectrum of a nonmagnetic phase in the amount of $30 \%$ together with a ferromagnetic sextet referred to the bcc Fe. To explain this fact it is supposed that, along with the fcc $\mathrm{Cu}$ and the bcc Fe phases, a noticeable quantity of a nonmagnetic phase, most likely fcc Fe, is also deposited. The proximity of lattice constants for fcc Fe and fcc $\mathrm{Cu}$ phases favors this $[113,114]$. Crespo et al. [115] considered that the decrease of magnetization for $\mathrm{Fe}_{51} \mathrm{Cu}_{49}$ solution after its annealing at high temperatures, which was observed in the works $[114,116]$, was caused by the precipitation of a significant amount of $\mathrm{Fe}$ in the form of dissolved atoms or small clusters.

The degree of metastability of $\mathrm{Fe}_{100-x} \mathrm{Cu}$ s solution significantly depends on its composition and milling time. This has been studied for $\mathrm{Fe}_{60} \mathrm{Cu}_{40}$ by X-ray diffraction and Mössbauer spectroscopy in [117]. At the early BM stage sextets with $H_{i}=5.0,10.0$, and $35.0 \mathrm{~T}$ appeared. After $\mathrm{BM}$ for $80 \mathrm{~h}$, a wide single sextet with $H_{i} \approx 25.0 \mathrm{~T}$ was

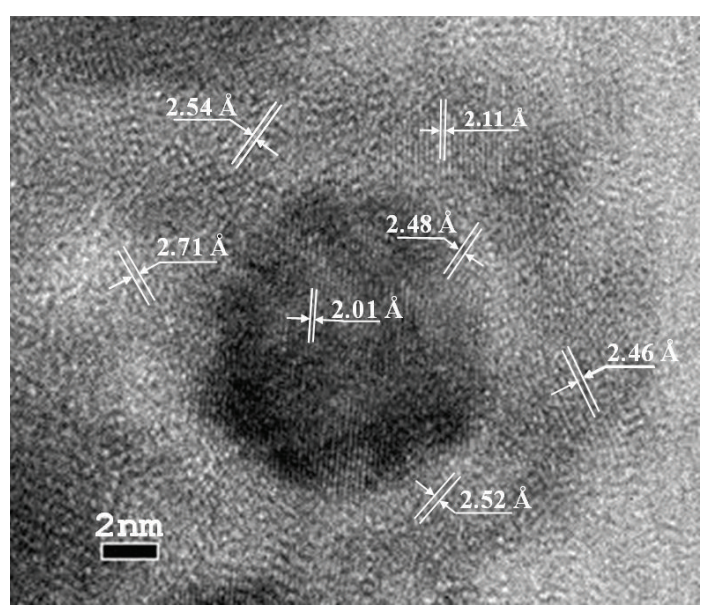

Figure 31: HRTEM pattern for FeCu (50.4 at.\%) particle after ageing in ambient air for 2 years. Interplanar spacings are pointed by arrows.

formed whereas the sextet related to bcc Fe almost completely disappeared.

So, there are a number of contradictions in different studies of structure and decomposition of metastable FeCu alloys concerning, in particular, the role of the high-temperature fcc Fe modification. An attempt to clarify these discrepancies based on studies of the structure and the magnetic state of aerosol $\mathrm{FeCu}$ nanoparticles in a wide composition range has been undertaken in a work [106]. Distinct features of the gas evaporation technique are a possibility to quench a hightemperature homogeneous solution and a fast attainment of the equilibrium state owing the atoms to diffuse into immiscible components as the particles are separated during their cooling in the inert gas atmosphere. The preliminary results for aerosol $\mathrm{FeCu}$ (50.4 at.\%) particles have been published in [118].

FeCu alloys containing 0.6-92.1 at.\% Cu were produced by direct alloying of the pentacarbonyl iron and electrolytic copper, using a $10 \mathrm{~kW}$ RF generator with a working frequency of $440 \mathrm{kHz}$ and a specially designed inductor. Aerosol particles were produced by fast evaporation of the predegassed in vacuum initial alloys in an Ar atmosphere at 133,400 , and 1330 Pa pressure from a tungsten spiral-basketshaped filament heated by ac power.

A specific feature of the technique applied for producing aerosol $\mathrm{FeCu}$ particles is that, arising near to the evaporator, they form a homogeneous solution. The subsequent fast quenching of particles in a rather cold inert gas leads to their segregation, which completes at elevated temperatures. Particles appear to be close to the equilibrium state because the atom diffusion and thermal exchange proceed quite fast owing to a smallness of their size. Due to the higher melting point of iron, when the formed particles begin cooling down as they move away from the evaporator, the solid iron core appears first, and the liquid copper shell covers it and later solidifies. Such structure is well seen in Figure 31 for $\mathrm{FeCu}$ (50.4 at.\%) particles. Therefore, one can conclude that the $\mathrm{bcc}$ and fcc phases observed in the XRD patterns for $\mathrm{FeCu}$ 


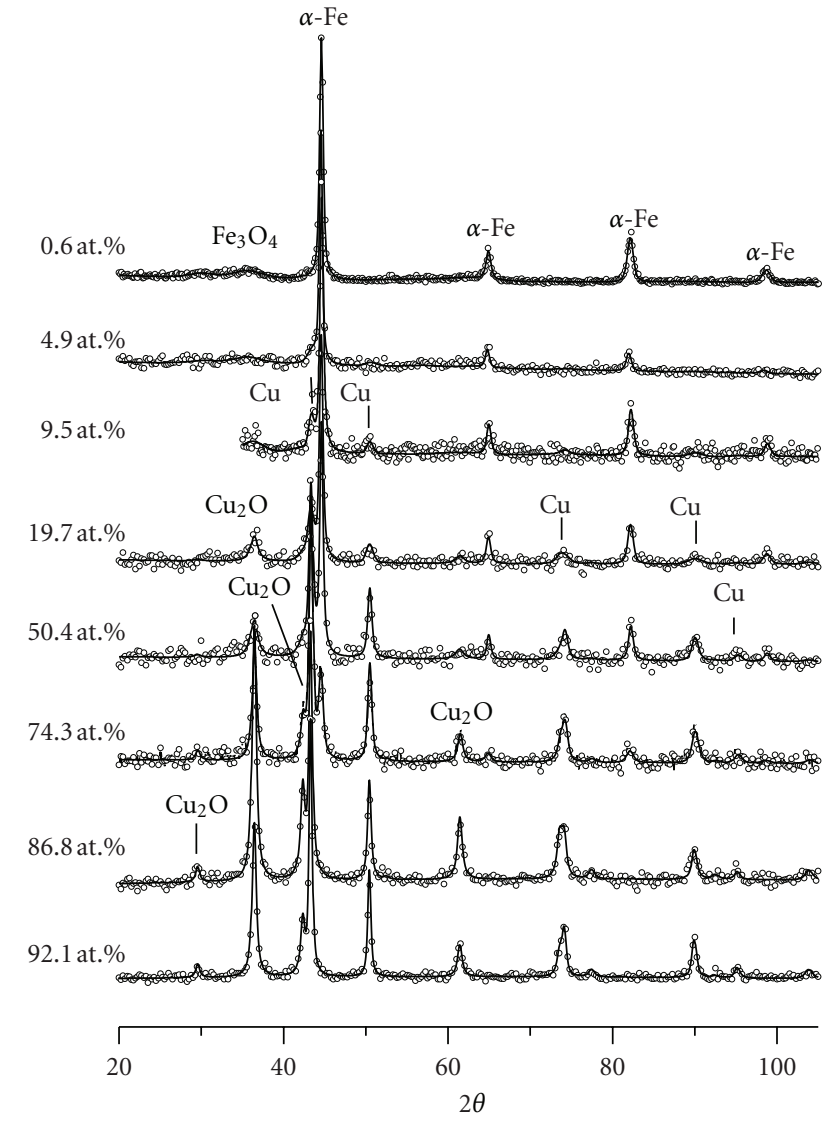

FIgURE 32: XRD patterns for $\mathrm{FeCu}$ particles with a various $\mathrm{Cu}$ content.

powders with $\mathrm{Cu}$ content from 4.9 to 74.3 at.\% are coexisting in the same particle. As the $\mathrm{Cu}$ content in $\mathrm{FeCu}$ particles grows up to 80 at.\%, their aerosol iron core decreases as far as it fully disappears, leading to ferromagnetism loss in the $\mathrm{FeCu}$ (86.8) (Figure 36(h)) and $\mathrm{FeCu}$ (92.1 at.\%) (Figure 36(i)) samples. In the course of producing $\mathrm{Cu}$-rich $\mathrm{FeCu}$ particles both Fe clusters and, mainly, copper grains appear. The iron dimers-trimers might be incorporated in the copper grains, resulting in a little increase of the fcc lattice constant of the $\mathrm{FeCu}(84.6$ at.\%) and $\mathrm{FeCu}$ (92 at.\%) samples.

The main measurements were performed with particles prepared at $p=400 \mathrm{~Pa}$. They have a complicated core-shell structure. This is evidenced by electron microscopy images and Fourier analysis for $\mathrm{FeCu}(50.4$ at.\%) particles after 2 years of exposure to air (Figure 31) $[106,118]$. The core has a size of about $12 \mathrm{~nm}$. The size of the whole particle together with the shell is $\approx 24 \mathrm{~nm}$. The shell is composed of crystallites of copper, $\mathrm{CuO}$, and $\mathrm{Fe}_{3} \mathrm{O}_{4}$.

Figure 32 shows the XRD patterns for as-prepared $\mathrm{FeCu}$ particles of different composition. It is seen that for the $\mathrm{Cu}$ diluted $\mathrm{FeCu}$ particles there are lines corresponding to the bcc phase of iron $(\alpha-\mathrm{Fe})$, which gradually decrease to their complete disappearance for $\mathrm{FeCu}$ (86.8 at.\%) particles. In the case of $\mathrm{FeCu}(0.6$ at.\%) particles there is also a diffuse peak due to $\mathrm{Fe}_{3} \mathrm{O}_{4}$. With increasing $\mathrm{Cu}$ content, lines relative to an fcc phase appear in the XRD patterns. First, it is as a

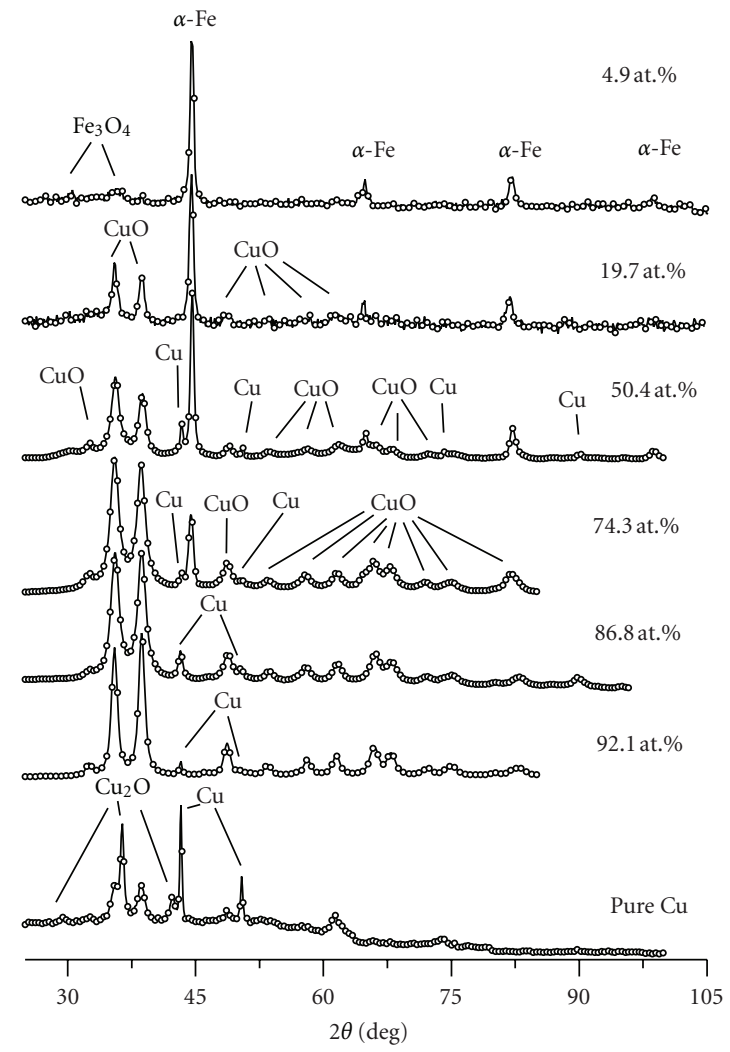

FIGURE 33: XRD patterns for $\mathrm{FeCu}$ particles with a various $\mathrm{Cu}$ content after ageing in ambient air for 2 years.

small asymmetry at the base line of the $\mathrm{Fe}(110)$ peak toward smaller Bragg angles in the $\mathrm{FeCu}$ (4.9 at.\%) sample. This asymmetry is converted to a separated peak $\mathrm{Cu}(111)$, when the copper content reaches 9.5 at.\%, and the latter continues growing with the $\mathrm{Cu}$ content. Its position as well as those of other lines emerging in the XRD patterns fits well with the fcc $\mathrm{Cu}$ lattice. In addition, the XRD patterns show the presence of lines relative to $\mathrm{Cu}_{2} \mathrm{O}$. The traces of iron oxide cannot be identified for all the samples containing more than 9.5 at.\% $\mathrm{Cu}$ against the background of the $\mathrm{Cu}_{2} \mathrm{O}$ lines.

After ageing the samples for 2 years in ambient air, the XRD patterns vary significantly (Figure 33). The amount of copper decreases, $\mathrm{Cu}_{2} \mathrm{O}$ disappears, and strong lines of $\mathrm{CuO}$ arise. It is curious that heating $\mathrm{FeCu}$ (50.4 at.\%) particles for $0.5 \mathrm{~h}$ in vacuum at $500^{\circ} \mathrm{C}$ leads to the reverse transformation of $\mathrm{CuO} \rightarrow \mathrm{Cu}_{2} \mathrm{O}$ as well as the appearance of intense lines of $\mathrm{Fe}_{3} \mathrm{O}_{4}$ and the decrease of iron content [118].

The concentration dependence of the crystallite size for the bcc and fcc phases, determined from line broadening with the help of the MAUD program, is shown in Figure 34. Subject to the complicated structure of particles, their mean sizes determined by XRD and TEM conform to each other. The composition dependence of the lattice constant for the bcc and fcc phases of the FeCu particles is shown in Figure 35 for two series of samples prepared in the identical conditions. Solid lines are drawn for the sake of clearness. The position of the bcc phase line for $\mathrm{FeCu}(<10$ at.\%) samples is not 


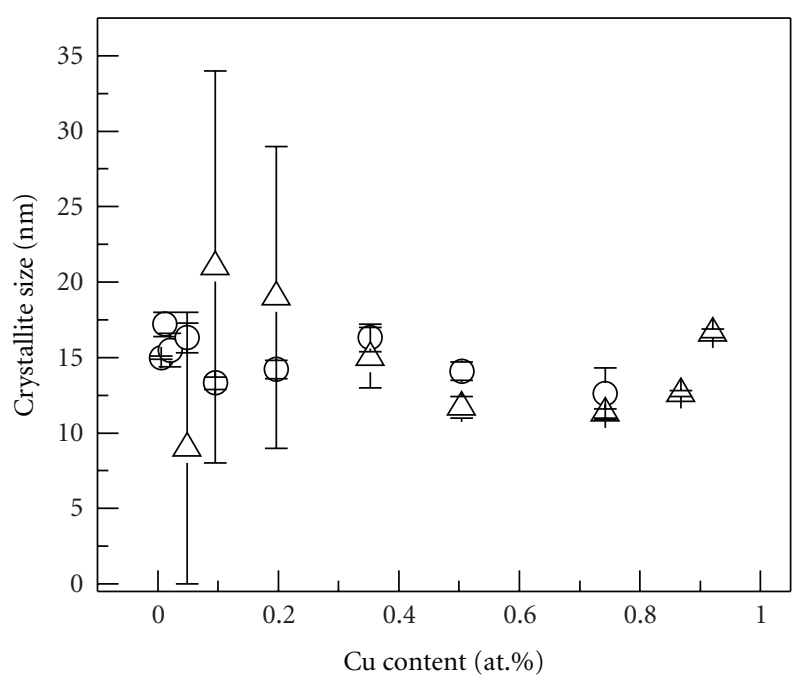

O bcc

$\Delta \mathrm{fcc}$

Figure 34: Concentration-dependent crystallite size for the bcc and fcc phases in $\mathrm{FeCu}$ nanoparticles.

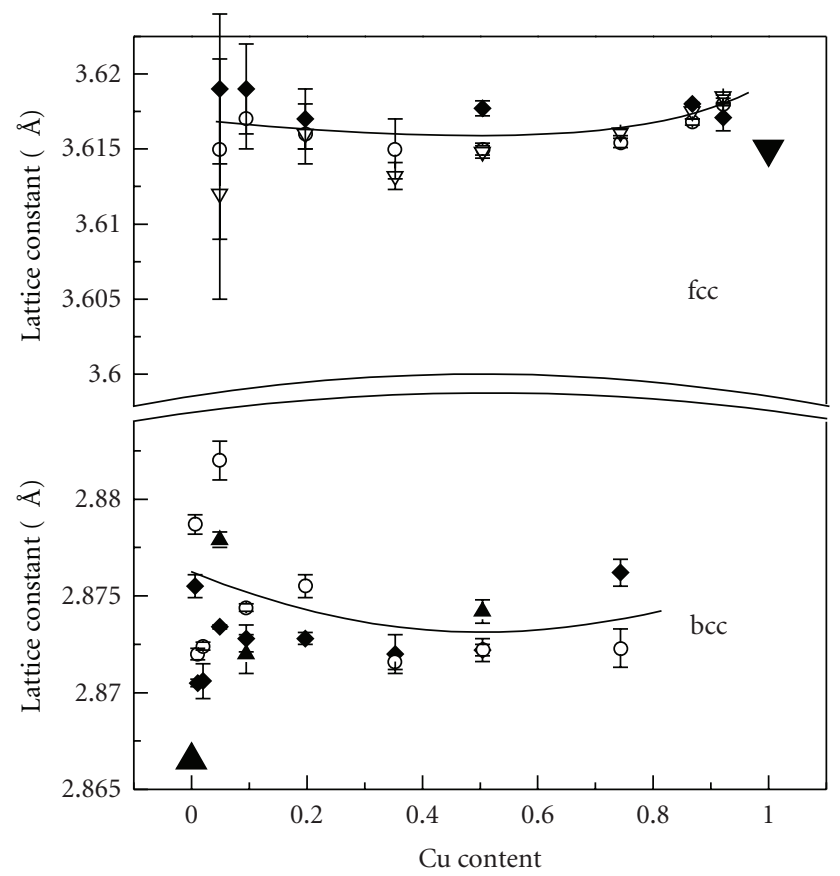

Figure 35: Concentration-dependent lattice constant for the bcc and fcc phases in FeCu nanoparticles. $\mathbf{\Delta}$-bulk Fe; $\mathbf{\nabla}$-bulk Cu.

sufficiently precise because of the large spread of data. In the case of the bcc phase the lattice constant significantly exceeds that for iron, whereas the lattice constant of the fcc phase is almost exactly equal to that for copper (Figure 35). This is likely due to the fact that the copper atoms are larger than iron atoms and as a result the embedding of copper atoms into the iron structure leads to an increase in the lattice parameter of the bcc phase. This increase is restricted quantitatively due to solubility limit of copper in the iron

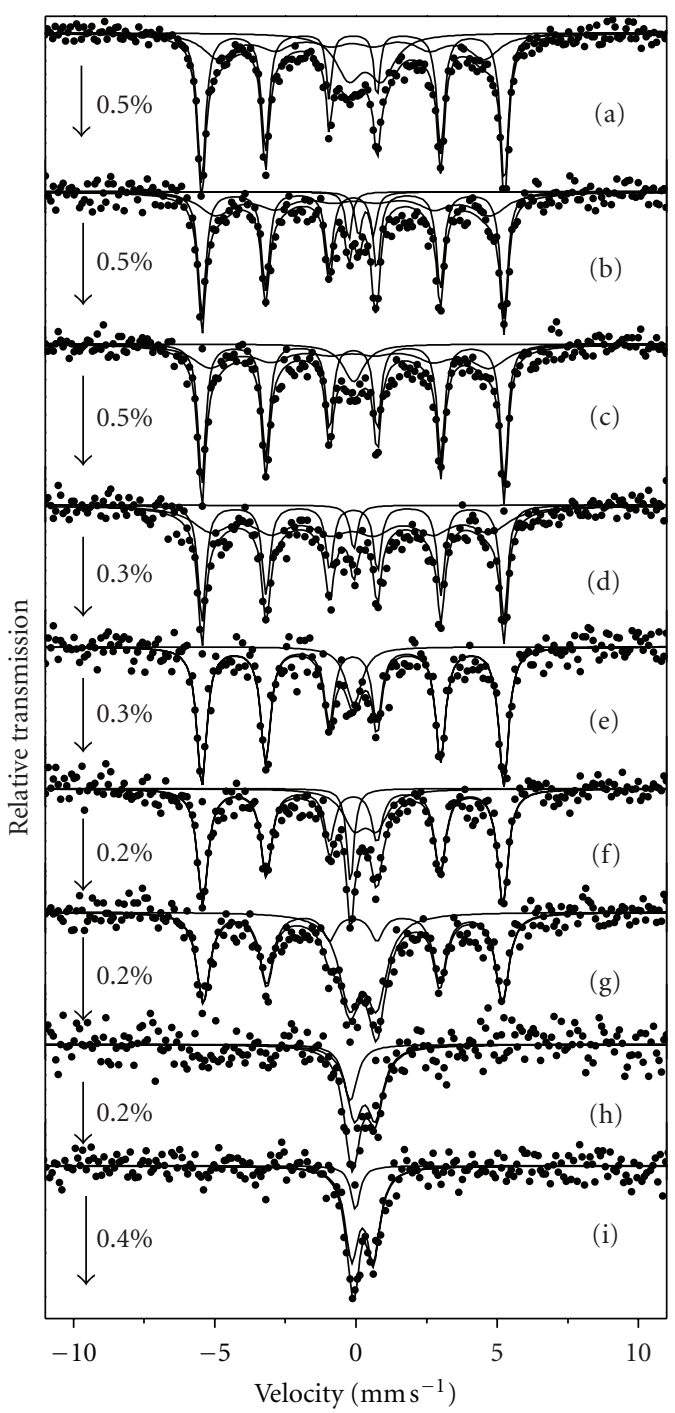

Figure 36: Room temperature Mössbauer spectra for $\mathrm{FeCu}$ particles with various $\mathrm{Cu}$ contents (at.\%). (a) 0.6; (b) 4.9; (c) 9.5; (d) 19.7 ; (e) 35.3 ; (f) 50.4 ; (g) 74.3 ; (h) 86.8 ; (i) 92.1 .

core of the particles. Some lift of the lattice constant curve for the fcc phase at the $\mathrm{FeCu}(>80$ at.\%) samples (Figure 35) denotes a slight embedding of the iron atoms into the $\mathrm{Cu}$ grains as well. In this case, the amount of iron atoms is not enough for the emergence of fragments of the bcc structure in the course of aerosol particles formation as evidenced by the lacking of the corresponding lines at the XRD patterns. On the other hand, the copper atoms, linking each other in $\mathrm{Ar}$, form aerosol particles with a fcc structure, inside and/or on the surface of which there are the aggregations of iron atoms. The latter, pushing apart the copper atoms, likely lead to some increase of the fcc lattice parameter for the $\mathrm{FeCu}$ (>80 at.\%) samples.

Figures 36 and 37 show how the room temperature (RT) and $80 \mathrm{~K}$ Mössbauer spectra of as-fresh $\mathrm{FeCu}$ samples change with $\mathrm{Cu}$ content. All spectra have a complex structure. For samples with $\mathrm{Cu}$ content from 0.6 to 19.7 at.\% (Figure 36) 


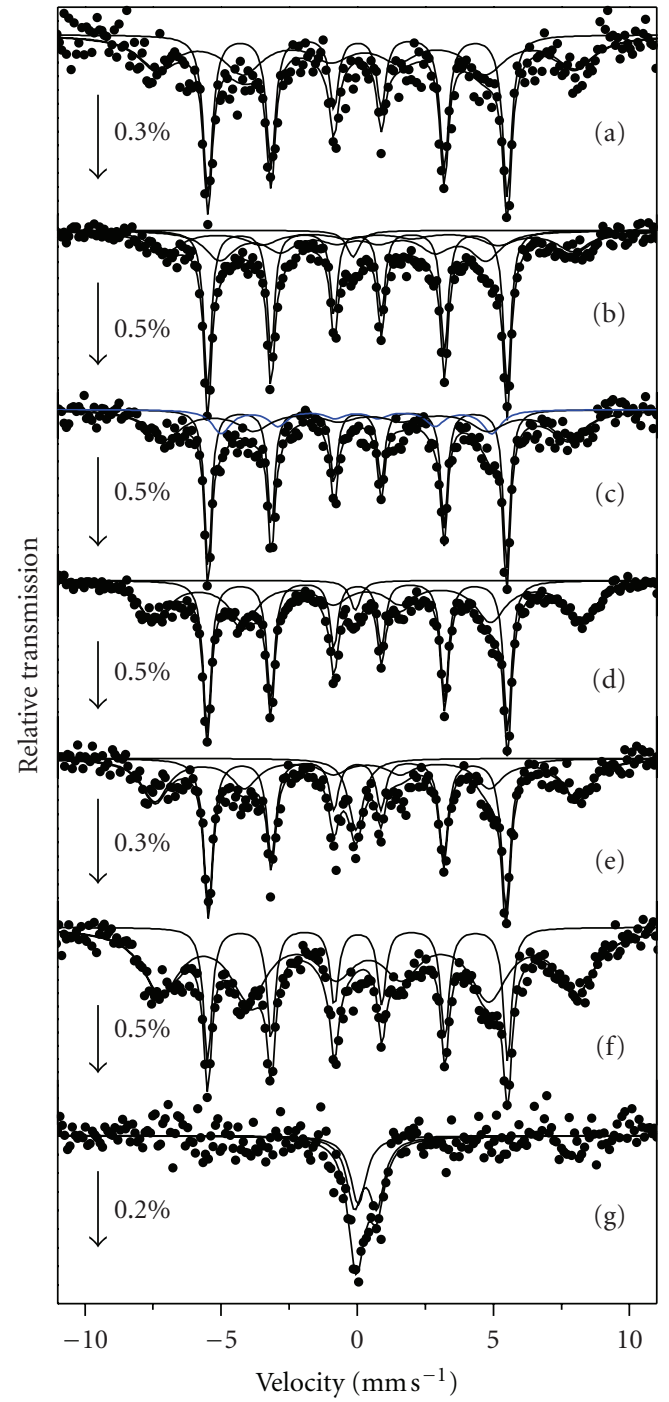

Figure 37: $80 \mathrm{~K}$ Mössbauer spectra for $\mathrm{FeCu}$ particles with various Cu content (at.\%). (a) 0.6; (b) 4.9; (c) 19.7; (d) 35.8; (e) 50.4; (f) 74.3 ; (g) 92.1 .

the observed sextet can be presented by superposition of two components, one of which with the hyperfine field $H_{i} \approx 33.0 \mathrm{~T}$ and the fraction area $S=49.1-56.7 \%$ belongs to the iron and another one with $H_{i} \approx 30.0 \mathrm{~T}(S=29.2-$ $34.5 \%$ ) (hereinafter we will name it as the second sextet) the origin of which will be considered below. In addition, the central part of the spectra changes from sample to sample. For $\mathrm{FeCu}(<4.9$ at.\%) samples the central part of spectra contains a doublet referred to $\mathrm{Fe}_{3} \mathrm{O}_{4}$. Here, unlike aerosol nanoparticles of pure iron, occurrence of oxide doublet, certainly, is connected with the small additive of copper in a surface layer of particles. A small singlet arise in the $\mathrm{FeCu}$ (4.9 at.\%) spectrum which holds also in the spectra of $\mathrm{FeCu}$ samples with higher $\mathrm{Cu}$ content. The doublet cannot be separated in spectra of $\mathrm{FeCu}$ (9.5 at.\%) to $\mathrm{FeCu}$ (35.3 at.\%) samples, though their $80 \mathrm{~K}$ spectra show sextet with $H_{i} \approx$ 48.0 T specific to $\mathrm{Fe}$ oxide.
The paramagnetic peak in the Mössbauer spectra of metastable $\mathrm{FeCu}$ alloys with a large $\mathrm{Cu}$ content has been a subject of many studies $[110,119,120]$. It is often connected with the presence of a high-temperature fcc iron modification which should be paramagnetic at room temperature. In view of affinity of crystal structures of copper and fcc Fe modification Weiss' model [39-41], which was repeatedly mentioned above, may be relevant to $\mathrm{FeCu}$ system.

It has been experimentally established that small iron particles precipitating from a oversaturated solution in copper [121-123] or fcc Fe thin films epitaxially grown-up on a surface of $\mathrm{Cu}$ single crystal [124-127] were paramagnetic $(\mathrm{PM})$ at room temperature and the transition to an AF state occurred below the Neel' point, that is, about $65-67 \mathrm{~K}$. The AF fcc Fe possessed a hyperfine field of $\approx 2.0 \mathrm{~T}$ and the magnetic moment per atom of $\approx 0.7 \mu_{B}$.

Klein et al. [110] have defined optimum conditions for the precipitation of the fcc Fe from metastable CuFe (3 at.\%) solutions produced by alloying the components in a quartz sealed-off tube. The prepared alloy was rolled to a thin foil, annealed to homogenize it in a high vacuum at $990^{\circ} \mathrm{C}$, and quenched in an ice water. A Mössbauer spectrum of the sample showed a central asymmetric peak which could be decomposed onto three components with different isomer shifts, namely: (1) singlet relative to a dominant number of Fe atoms in the solution; (2) doublet referred to Fe clusters with a different environment; (3) singlet related to the fcc Fe. The larger amount (90\%) of the fcc Fe was formed after the foil annealing at $450-600^{\circ} \mathrm{C}$ for $48 \mathrm{~h}$. Mössbauer spectra of multilayer films formed by Fe layer of $0.5 \mathrm{~nm}$ thick between $5 \mathrm{~nm} \mathrm{Cu}$ layers for two samples of different laboratories were compared [120]. The results for both samples did not, in principle, differ. Besides the Fe oxide doublet, the PM peak also included two components one of which was transformed at $4.2 \mathrm{~K}$ to a sextet with $H_{i} \approx 27.0 \mathrm{~T}$ whereas another remained as a singlet. The former was ascribed to Fe atoms in a bcc-like coordination. Spreading of the singlet at $4.2 \mathrm{~K}$ has allowed one to assume that this component is connected with the fcc Fe because the hyperfine field for the AF fcc Fe below $T_{N}$ is approximately $2.4 \mathrm{~T}$.

On the other hand, Window [111] and Keune et al. [109] doubted if the fcc Fe clusters would be able to appear in a strongly diluted metastable CuFe solution because they should be in the AF state at $4.2 \mathrm{~K}$ and the corresponding spread singlet would have a too low hyperfine field $(\approx 2.5 \mathrm{~T})$ compared with that of $8.0 \mathrm{~T}$ for the observed one. The results of work [106] show that the PM fcc Fe in Cu-rich $\mathrm{FeCu}$ systems appears only after heat treatment resulting in coarsening the Fe aggregations inwards or on the surface of $\mathrm{Cu}$ grains.

The existence of an FM fcc Fe state and its role in the behavior of the $\mathrm{FeCu}[110,128]$ is difficult to understand as this state should remain at room temperature. This FM state in fcc iron has been firstly revealed by extremely rapid quenching of aerosol Fe particles of $50 \mathrm{~nm}$ diameter [43].

In the case of epitaxial growth of Fe films on a $\mathrm{Cu}$ single crystal, there are favorable conditions for the formation of fcc Fe because of the similarity of crystal structures. 
Gradmann and Isbert [129] prepared fcc Fe(111) films of 2 or 3 atomic layers by epitaxial growth on the surface of a thin $\mathrm{Cu}_{1-x} \mathrm{Au}_{x}(111)$ crystal. The lattice parameter of the latter could be modified by changing Au content. Such fcc Fe films showed a gradual transition from weak ferromagnetism $\left(0.6 \mu_{B}\right.$ /atom, lattice constant $\left.a=3.615 \AA\right)$ when deposited on a clean $\mathrm{Cu}(111)$ surface to a strong ferromagnetism $\left(2.6 \mu_{B} /\right.$ atom, $\left.a=3.68 \AA\right)$ with a sufficient addition of gold (14 at.\%). Keune et al. [127] also deposited epitaxially iron on a $\mathrm{Cu}$ single crystal and they found that both FM and AF states of fcc Fe were observed, depending on the deposition conditions. By choosing the substrate structure they could vary the lattice constant of the epitaxial fcc Fe film, the latter was AF at $a \leq 3.63 \AA$ and FM at $a \geq 3.67 \AA$.

In a recent paper [130] the results of numerous studies on the epitaxial growth of iron films on the surface of $\mathrm{Cu}(001)$ at room temperature have been summarized, and the following generalizations have been supposed. Up to four monolayers the Fe film is FM overall with the easy axis perpendicular to its plane (the 1st level). For the layers between the 5th and 10th the easy axis is still perpendicular to the film plane but only the first layers are FM whereas the other layers show AF ordering (the 2nd level). The follow-up layers form FM bcc iron with the magnetization direction in the film plane (third level). There is no consensus whether the first level layers and the initial layers of the 2 nd level constitutes a bcc-like or shear-deformed fcc structure. The rest layers of the 2nd level present everywhere the AF fcc structure, as confirmed by measurements with the two-photon and inverse photoemission methods. Thus, in terms of electronic structure, just thin enough fcc Fe precipitates on $\mathrm{Cu}(001)$ may be FM, whereas the subsequent thicker layers of fcc Fe should be AF. However, the thickness of the layer at which the fcc Fe undergoes FM $\rightarrow$ PM transition remains ambiguous.

Comparing the results of the aforecited works we can conclude that the magnetic state of thin fcc Fe films epitaxially grown on different faces of $\mathrm{Cu}$ single crystals at room temperature varies considerably. It can be either PM [(111) face] or FM [(001) face] despite the fact that in both cases, the atomic volume remains unchanged.

The issue of the possible presence of FM fcc Fe in the $\mathrm{FeCu}$ system has been raised repeatedly in the literature $[8,109,129-132]$. It was tried to obtain by heat treatment of ball-milled (BM) both pure iron and iron in a mixture with copper. BM Fe powder with the grains size of $10 \mathrm{~nm}$ was examined by Mössbauer spectroscopy, HRTEM, and magnetization measurements [132]. It was found that a new phase appeared after annealing at $570 \mathrm{~K}$ for $1 \mathrm{~h}$ at the expense of diminishing the bcc Fe. The latter had a hyperfine field of $21.0 \mathrm{~T}$ and lower magnetic moment than that for a bulk iron. A magnetic order-disorder transition at this phase was observed at $\sim 500 \mathrm{~K}$ and it was ascribed to the fcc Fe. It was assumed that the bcc and fcc phases were in an orientation relation, namely, $(110)_{\mathrm{bcc}}$ and $(111)_{\mathrm{fcc}}$. In a work [133] the $\mathrm{BM}$ fcc $\mathrm{Fe}_{16} \mathrm{Cu}_{84}$ alloy was heated at different temperatures lower than $1100 \mathrm{~K}$ followed by cooling to room temperature. Neutron diffraction and magnetic studies of the sample were carried out during each cycle of heat treatment. By cooling from $1100 \mathrm{~K}$, in addition to bcc $\mathrm{Fe}$ and $\mathrm{fcc} \mathrm{Cu}$ phases, there appeared about $5 \%$ of isolated fcc Fe precipitates. Subsequent heating at above $600 \mathrm{~K}$ led to an increase of the magnetization by $50 \%$ compared with its value at room temperature. According to the Weiss' model the reason for the hightemperature anomalies of magnetization was seen in the thermal excitation of FM state with respect to the ground AF state of the fcc Fe. The thermal expansion measurements showed that fcc Fe lines were retained when cooling the sample to a temperature of about $650 \mathrm{~K}$.

Extrapolating these data to room temperature gave the lattice constant for the PM fcc Fe $a=3.59 \AA$. In some cases, a splitting of sextets into two components with the hyperfine fields 33.0 (bcc Fe) and 21.0-28.0 T was observed in the Mössbauer spectra of the Fe-rich FeCu alloys [5, 113, 119]. The emergence of the second component was ascribed to the different environments of iron atoms surrounded by copper atoms according to a binomial distribution. In this context, the possibility of formation of FM fcc Fe at the interface of copper and iron was ignored. On the other hand, the fcc Fe apparently plays an important role in the Invar effect observed at the $\mathrm{BM}$ metastable fcc $\mathrm{Fe}_{44} \mathrm{Cu}_{56}$ alloy [12]. The lattice parameter measured by neutron diffraction in the temperature range between 300 and $350 \mathrm{~K}$ remains almost unchanged. At $350 \mathrm{~K}$, it sharply increases which is typical for Invar effect. Upon heating the alloy above $350 \mathrm{~K}$ it undergoes the $\mathrm{PM} \rightarrow$ FM transition. The two-state Weiss' model satisfactorily explains the behavior of the Invar $\mathrm{Fe}_{44} \mathrm{Cu}_{56}$ alloy $[12,133]$.

In our work [106] both states of fcc Fe are clearly manifested in the Mössbauer spectra of Fe-rich FeCu systems in the form of a second sextet with a hyperfine field of $H_{i}$ $\approx 30.0 \mathrm{~T}$ (FM fcc Fe) and a concomitant singlet attributed to PM fcc Fe. Indeed, this singlet coexists and probably gradually disappears together with the second sextet with increasing $\mathrm{Cu}$ content in the sample from 4.9 to 35.3 at.\%. Their disappearance in the $\mathrm{FeCu}(35.3$ at.\%) sample is caused by an increased degree of surface oxidation of copper grains (Figure 32), which prevents the formation of fcc Fe in the interface area between the iron core and the copper shell. The same reason explains the disappearance of the second sextet and the concomitant singlet after 2-year ageing of the $\mathrm{FeCu}$ ( 4.9 at.\%) sample in air.

The singlet in the Mössbauer spectrum of the $\mathrm{FeCu}$ (35.3 at.\%) sample (Figure 36(e)) is due to another reason, namely, to the presence of bcc Fe aggregations. Such aggregations have little or not at all in the $\mathrm{FeCu}(<35.3$ at.\%) samples. Otherwise, they would have manifested themselves most notably as a singlet in the $\mathrm{FeCu}(0.6$ at.\%) sample which does not correspond to reality (Figure 36(a)).

From the measured Mössbauer spectra, one may suppose that the Fe layers closest to the copper surface have an FM fcc structure responsible for the appearance of the second sextet with a hyperfine field of $H_{i} \approx 30.0 \mathrm{~T}$ (Figures 36(a)-36(d)). Ferromagnetism of the fcc Fe layer can be explained by the fact that the surface of copper apparently faced to the iron with the (001) facet.

The layers being more distant from the copper surface might be PM, giving a central singlet in the spectrum (Figures 36(b)-36(d)). The behavior of Fe atoms in the 
particle border zone is rather complicated, because they are exposed to the simultaneous action of the $\mathrm{Cu}$ shell and Fe core. The Fe atoms acquiring the fcc $\mathrm{Cu}$ structure $(a=$ $3.615 \AA$ ) have the volume per atom equal $11.81 \AA^{3}$, whereas in the bcc structure (Figure $35, a=2.873 \AA$ ) the volume occupied by the Fe atom is $11.86 \AA^{3}$. Thus, two different structures with the almost equal atomic volumes are FM. The appearance of the PM layers in the interstitial region can be attributed to the deviation of the atomic volume from the border value. FM fcc Fe adjacent to the $\mathrm{Cu}$ layer takes its lattice constant $a \approx 3.615 \AA$ (note that epitaxial fcc Fe films with this value of lattice parameter have to be PM [127]). If one takes into account that the bcc Fe packing is looser, hence, it has a weaker effect on the atoms in the transition region than the close-packed fcc Fe structure. Therefore, the latter must also hold in layers more distant from the $\mathrm{Cu}$ surface. However, the influence of the $\mathrm{Cu}$ surface becomes weaker in these layers and the fcc Fe lattice constant will decrease, tending to its intrinsic value at room temperature, namely, $a=(3.57-3.59) \AA$ [the respective atomic volume (11.38-11.57) $\AA^{3}$ ], which corresponds to the PM state of the fcc Fe. Thus, the appearance of the singlet in the Mossbauer spectrum corresponds to a decrease in the atomic volume in the intermediate layer, which is not larger than $0.43 \AA^{3}$.

When the $\mathrm{Cu}$ layer covering the Fe core of the particle is thin, the $\mathrm{Cu} \mathrm{fcc} \mathrm{structure} \mathrm{is} \mathrm{not} \mathrm{observed} \mathrm{in} \mathrm{the} \mathrm{XRD}$ patterns, as in the case of the $\mathrm{FeCu}$ (0.6 at.\%) sample (Figure 32). Nevertheless, there is a sextet with a hyperfine field $H_{i}=29.2 \mathrm{~T}$ in the Mössbauer spectrum (Figure 36(a)), with a singlet being absent. Hence, a thin $\mathrm{Cu}$ layer is unable to keep the sufficiently removed Fe atoms in the PM fcc structure. But such a singlet appears in the $\mathrm{FeCu}$ (4.9 at.\%) spectrum (Figure 36(b)) together with the sextet respective to the FM layer fcc Fe. This singlet remains when cooling the sample to $80 \mathrm{~K}$ (Figure 37 (b)) in accordance with the fact that the $T_{N}$ for the AF fcc Fe is equal to $65-67 \mathrm{~K}$.

Comparing the above data for aerosol particles and the corresponding data obtained by other methods for the metastable $\mathrm{FeCu}$ alloys, one can draw the following conclusions. In all cases there was a region of two-phase composition of samples in a wide range of $\mathrm{Cu}$ content. However, the boundaries of this region were very fuzzy. Thus, according to Keune et al. [109], metastable solid $\mathrm{Fe}_{100-x} \mathrm{Cu}_{x}$ solutions, produced by the joint condensation of the Fe and $\mathrm{Cu}$ vapors, with the copper content $x=0-40$ at.\% have a bcc structure, for $x=40-65$ at. $\%$ the solution consists of a mixture of bcc and fcc structures, whereas for $x>65$ at. $\%$ a sole fcc structure was only detected. On the other hand, $\mathrm{Fe}_{100-x} \mathrm{Cu}_{x}$ mixtures after BM for $52 \mathrm{~h}$ had fcc structure for $x \geq 50$ at. $\%$ and a bcc for $x \leq 20$ at.\% [134]. Both the bcc and fcc phases were principally FM for $x \leq 80$ at. $\%$ and the sample with $x=90$ at. $\%$ remained PM down to $78 \mathrm{~K}$.

In our work [106], coexistence of bcc and fcc phases was observed at $x=4.9-74.3$ at.\%, with the samples being everywhere FM (Figure $36(\mathrm{~g}) ; H_{i} \approx 33.0 \mathrm{~T}$ ). At a higher $\mathrm{Cu}$ content the Mossbauer spectra showed only the central PM peak (Figures 36(h) and 36(i)) presented by superposition of a singlet and a doublet, the shape of these spectra remained down to $80 \mathrm{~K}$ (Figure $37(\mathrm{~g})$ ). The separated singlet, apparently, cannot be associated with the PM fcc Fe because in such a case it would have to appear together with the FM sextet related to the fcc Fe which is lacking. In our opinion, the singlet might be referred to the small aggregations of $\mathrm{Fe}$ atoms and the doublet to Fe clusters with a distorted cubic symmetry due to their interaction with the fcc $\mathrm{Cu}$ lattice.

The second sextet and the concomitant singlet observed in the Fe-rich $\mathrm{FeCu}$ systems are most likely due to the fcc $\mathrm{Fe}$, existing in the transition region from the $\mathrm{Cu}$ shell of the $\mathrm{Fe}$ core in the particles. The explanation assuming a model of filling the various sites of coordination spheres at a resonating $\mathrm{Fe}$ nucleus with $\mathrm{Cu}$ atoms, as suggested in a number of the above cited works $[5,113,119]$ is not plausible. Indeed, the area ratio of the second sextet and the sextet related to Fe is equal to about 0.6 for all samples (Figure 36). This means that more than a half of the Fe atoms must have a copper atom at its first coordination sphere, which is not feasible because of the low solubility limit of copper in iron. In addition, the presence of many $\mathrm{Cu}$ atoms contributes to their association, thereby violating the basic assumption of independent random distribution of impurity atoms in the Fe lattice $[5,112,119]$.

In general, the aerosol $\mathrm{FeCu}$ particles are evidently in a stable mechanical combination of copper and iron crystallites (grains). The lattice parameter of the latter is slightly larger due to embedding of $\mathrm{Cu}$ atoms into $\mathrm{Fe}$ lattice. One might think that the $10-30 \mathrm{~nm}$ FeCu particles obtained by joint condensation or BM method have a similar structure. If so, the widespread notion of "metastable alloy" as the nonequilibrium solution is not entirely correct with respect to the $\mathrm{FeCu}$ system. For example, in experiments with granular films one assumed that the alloying of the components, practically insoluble in each other, occurs at the atomic level with the formation of metastable FeCu alloy $[5,135-139]$. In fact, it is probably not the case. The final result of BM, apparently, is the formation of small regions (grains), in which $\mathrm{Fe}$ and $\mathrm{Cu}$ are in an intimate mechanical contact, with the copper atoms being embedded into the Fe lattice. In the case of aerosol particles, the Fe core with embedded $\mathrm{Cu}$ atoms could be taken as a genuine equilibrium solution, and we can assume it as a new bcc phase. However, the fcc phase consists of pure copper, the diffusion of $\mathrm{Fe}$ atoms into which does not manifest itself, at least until the $\mathrm{FeCu}(74.3$ at.\%) composition. A slight increase in the lattice constant at the $\mathrm{FeCu}(86.8$ at.\%) and $\mathrm{FeCu}$ (92 at.\%) samples is undoubtedly due to incorporating small iron complexes, likely dimmers-trimers, into the copper grains in the course of aerosol particle preparation.

\section{A Fine Structure in the Hyperfine Field Distribution (HFD) at ${ }^{57}$ Fe Nuclei}

Determination of hyperfine field distribution (HFD) at ${ }^{57} \mathrm{Fe}$ nuclei is one of the prime goals for Mössbauer spectroscopy of iron contained substances. For this purpose one applies a variety of codes, for instance, the NORMOS program [26] which offers fitting either by a superposition of components (SITE) or by a hyperfine distribution (DIST). In the last 
case spectra can be presented by superposition of up to 50 sextets. A contribution of every sextet is defined by finding the least-squares deviation $\left(\chi^{2}\right)$ of the fitted spectrum with respect to the measured one. As a rule, the hyperfine field range is chosen quite broad. Therefore a step for change of hyperfine field between the adjacent trial sextets $(\Delta)$ appears to be relatively large which might result in a loss of details in HFD.

Analyzing Mössbauer spectra of some diluted $\mathrm{Rh}_{1-x} \mathrm{Fe}_{x}(x=0.1,0.2$, and 0.3$)$ [140], $\left(\mathrm{Pd}_{100-x} \mathrm{Rh}_{x}\right)_{90} \mathrm{Fe}_{10}(x=0,10,20)[141], \mathrm{Pd}_{1-x} \mathrm{Fe}_{x}(x=0.03-$ $0.20), P d F e A g, P d F e R h$ [142] alloys taken at $T=5 \mathrm{~K}$, a hyperfine field distribution (HFD) at ${ }^{57} \mathrm{Fe}$ nuclei was shown to be discontinuous and expressed in occurrence of a wavy profile at the HFD. Authors of works [140-142] applied the own fitting program and explained their results by variations of contributions from the magnetic moments of iron atoms next to the resonating atom, assuming a competition of ferro- and antiferromagnetic interactions of neighbors and their random distribution in the nearest coordination sphere of iron. The calculation scheme close to that has been suggested in a work [103]. Though such a treatment of Mössbauer spectra became worldwide, it is completely useless at a comparable content of components or for ordered alloys due to violation of the random distribution of atoms in alloy. Moreover, as shown in [84] for FeCr alloy, the similar approach is not always applicable for diluted solid solutions too. It has been established that a split or a broadening of external lines of sextet for nanoparticles of pure Fe and of some Fe-rich alloys had been caused by quenching of a magnetic order of the high-temperature fcc phase [43].

5.1. On the Conditions Eliciting a Detailed Structure in HFD $a t{ }^{57} \mathrm{Fe}$ Nuclei. If one takes into account overlapping of lines of the ${ }^{57} \mathrm{Fe}$ source and absorber, then a natural linewidth in Mössbauer spectra of pure iron can be easy estimated as $\sim 0.2 \mathrm{~mm} \times \mathrm{s}^{-1}$. A linewidth observed in our measurements with iron typically was about $0.35 \mathrm{~mm} \times \mathrm{s}^{-1}$ that is by factor of about 1.8 larger than a total natural linewidth. This permits to suppose that the spectra may be a superposition of several sextets. A change of magnetic field by $1 \mathrm{~T}$ is connected with displacement in a Mössbauer spectrum $\Delta V\left(\mathrm{~mm} \mathrm{~s}^{-1}\right)$ and energy by the following relation:

$$
1 \mathrm{~T} \longrightarrow 0.323 \mathrm{~mm} \times \mathrm{s}^{-1} \longrightarrow 1.55 \times 10^{-8} \mathrm{eV} .
$$

Though the linewidths increase to some extent due to instrumental effects, they are evidently kept for every sample examined in standard conditions. So, except conventional Zeeman levels at ${ }^{57} \mathrm{Fe}$ nucleus, there are also slightly shifted levels resulting in occurrence of additional sextets in the spectrum.

If one would wish to reveal a fine structure in HFD for some magnetic component separated in a particular Mössbauer spectrum, it would be reasonable to apply all resources of the NORMOS code available for HFD analysis (up to 50 trial sextets) for describing it by superposition of trial sextets within a narrow hyperfine range. In this way one might set a comparatively small step $\Delta$ that would allow revealing a plausible fine structure in HFD. But for all that a question arises if the program NORMOS can generally distinguish two closely spaced narrow peaks in HFD. If so, what restrictions should be imposed on the defined parameters? From this standpoint, determination of HFD from Mössbauer spectra has been critically analyzed [143]. The results of this analysis have served as a basis for developing the technique, which previously had ensured the finding of several peaks spaced by $0.5-1.0 \mathrm{~T}$ in HFD for a bulk iron [144, 145] as well as for FePd [146] and FeCo [147] alloys.

The NORMOS code summarizes positive results of the previous theoretical works $[148,149]$. It permits, in particular, to introduce an arbitrary smoothing parameter $\lambda$ which Hesse and Rübartsch [148] introduced to smooth the ill-posed initial conditions for the integral equation coupling a detected Mössbauer spectra with a set of simulated spectra.

A role of smoothing parameter $\lambda$ at defining HFD from Mössbauer spectra as well as the meaning of criterion of the fitting goodness (Hesse-Rübartsch parameter) has been clarified in [143]. The latter is the ratio of square deviation of the calculated curve $\left(s_{i}\right)_{c}$ from the experimental one $s_{i}$ to the total count number $N_{\Sigma}$ in all channels of analyzer, namely,

$$
\mathrm{HR}=\left(N_{\Sigma}\right)^{-1} \sum_{i=1}^{N}\left[s_{i}-\left(s_{i}\right)_{c}\right]^{2}
$$

The best calculated result yields $\mathrm{HR}=1$. In this case the deviations of the obtained fit correspond to a statistical error of the number of counts. Basing on the general concepts of statistics, it has been shown that HR criterion is always applicable and not only to assess the effect of $\lambda$ value [143]. The best fitting quality is obtained by meeting the condition $\mathrm{HR} \leq 1$ when the standard deviation of the calculated values from the observables does not go beyond the statistical errors of measurements.

5.2. Discreteness in HFD for Pure Iron and Fe-Based Alloys. To reveal if a discontinuity of HFD is possible in iron-based alloys, FePd [146], FeCo [79, 147], and FeCr [150] bulk alloys and nanoparticles have been examined. The set of peaks in HFD separating from each other by $0.5-1.0 \mathrm{~T}$ has been found out in all three alloys.

In a work [146] it has been first shown that the discrete structure of HFD is inherent to bulk FePd alloys in a wide (from 2.2 to $91 \mathrm{wt} . \%$ ) Pd concentration range both at low and room temperatures. The similar pattern with some features takes place also in FePd nanoparticles with a size of $D \approx 12 \mathrm{~nm}$. Mössbauer spectra and corresponding HFD for FePd (2.2, 35, and 49.6\%) alloys and nanoparticles are shown in Figures 38-40.

The following features are appreciable at first sight. The quantity of peaks depends on Pd content in FePd alloy. Its number is maximal at a point of bcc $\rightarrow$ fcc transition for a massive FePd (35wt.\%) alloy when the spectrum lines strongly broaden (Figure 39). In this case HFD looks like two groups of peaks, one of which is located in intervals between others. In FePd (49.6\%) alloy spectrum, lines are split on two 


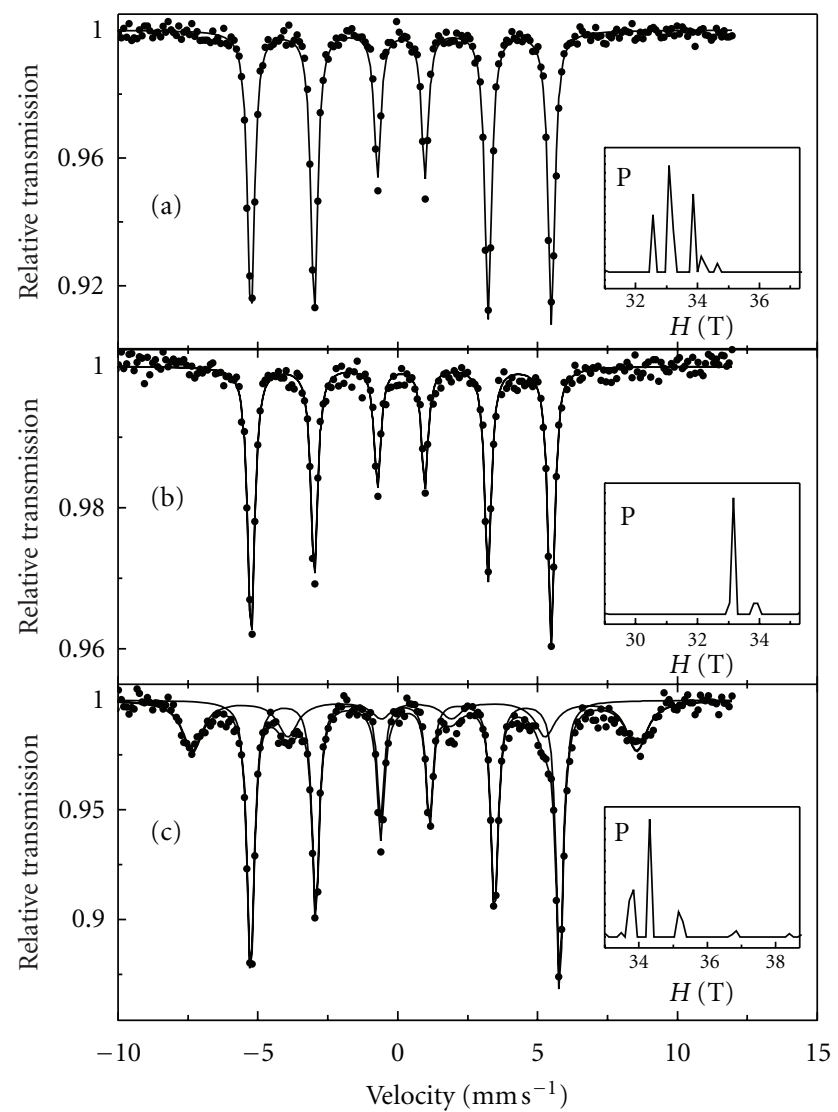

Figure 38: Mössbauer spectra for FePd (2.2\%). Hyperfine field distribution for a ferromagnetic component in spectrum is shown in inserts. (a) The bulk alloy, $T=293 \mathrm{~K}$; (b) particles with a size of $D=12 \pm 3 \mathrm{~nm}$ at $T=293 \mathrm{~K}$; (c) the same particles at $T=100 \mathrm{~K}$.

components, and accordingly HFD contains two groups by 3 peaks (Figure 40). At $T=293 \mathrm{~K}$ HFD for nanoparticles contains only $1-2$ peaks. With drop of temperature to $100 \mathrm{~K}$ some more 1-2 peaks are added to them.

Influence of a little additive to iron on the HFD shape was studied by comparison of spectra of an iron foil and $\mathrm{FeCr}$ (2.36 at.\%) alloy [150]. The results for pure iron (a foil and $13 \mathrm{~nm}$ particles) are given in Figure 41 and the corresponding data for $\mathrm{FeCr}$ (2.36 at.\%) alloy are presented in Figure 42.

A group of three peaks in HFD is observed for a massive iron (Figure 41(a)) irrespective of its origin (in addition, iron pentacarbonyl $\mathrm{Fe}(99.9+\%)$ foil was also examined) [144]. These peaks are referred to the effective hyperfine fields $H_{e} \approx$ $32.2,32.9$ and $33.5 \mathrm{~T}$. It is notable that the small Fe particles show only a single peak with $H_{e} \approx 32.6 \mathrm{~T}$, whereas at $T=80 \mathrm{~K}$ apart from the peak with $H_{e} \approx 33.9 \mathrm{~T}$, an additional peak with $H_{e} \approx 34.75 \mathrm{~T}$ arises (Figure $41(\mathrm{c})$ ). At $T=4.2 \mathrm{~K}$ position of these two peaks practically does not change. Call attention to absence of oxide traces in the spectra of small Fe particles at RT (Figure 41(b)). However at low temperatures a sextet with an average field $\left\langle H_{e}\right\rangle \approx 49 \mathrm{~T}$ appears in the spectra and it is related to $\mathrm{Fe}_{3} \mathrm{O}_{4}$ (Figures 41(c), and 41(d)). Basing on sextet areas, the oxide content is about $40 \%$. Thus, the initial X-ray amorphous and invisible in Mössbauer spectra

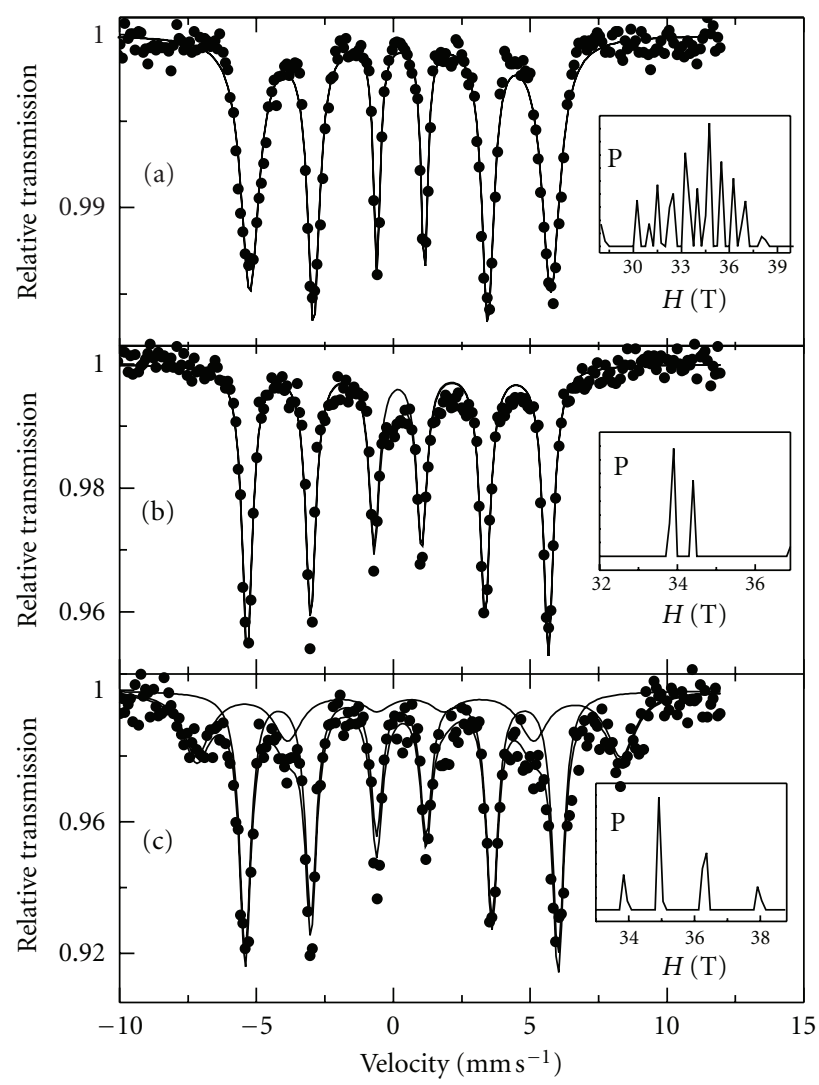

FIgure 39: Mössbauer spectra for FePd (35\%). Hyperfine field distribution for a ferromagnetic component in spectrum is shown in inserts. (a) The bulk alloy, $T=293 \mathrm{~K}$; (b) particles with a size of $D=12 \pm 3 \mathrm{~nm}$ at $T=293 \mathrm{~K}$; (c) the same particles at $T=100 \mathrm{~K}$.

oxide shell of small Fe particles becomes ferrimagnetic at low temperatures. Note that when defining HFD at ${ }^{57} \mathrm{Fe}$ nuclei of the metallic core of small particles a contribution of $\mathrm{Fe}$ oxide in their Mössbauer spectra was eliminated. At large Fe particles a presence of oxide was not observed in the Mössbauer spectra even at $4.2 \mathrm{~K}$ because of its small relative content. Appearance of two peaks $\left(H_{e}=33.9\right.$ and $\left.34.75 \mathrm{~T}\right)$ with cooling small particles to $80 \mathrm{~K}$ (Figure 41 (c)) occurs simultaneously with appearance and development of sextet in spectra related to $\mathrm{Fe}_{3} \mathrm{O}_{4}$ (a respective hyperfine field is $\left.H_{e}=46-49 \mathrm{~T}\right)$. Hence one may suppose that one of two peaks $\left(H_{i}=34.75 \mathrm{~T}\right)$ is caused with interaction between surface $\mathrm{Fe}$ atoms and oxide shell of the nanoparticles. After warming small particles from $80 \mathrm{~K}$ up to $293 \mathrm{~K}$, there is again a single peak but at somewhat higher value, namely, $H_{e}=$ $33.46 \mathrm{~T}$ instead of $H_{e}=32.6 \mathrm{~T}$. It means that the hyperfine field specific to the low temperature nearly holds, whereas the peak with $H_{i}=34.75 \mathrm{~T}$ likely caused with exchange interaction of the particle core with its shell vanishes in the Mössbauer spectrum together with disappearance of Fe oxide sextet.

The chrome additive to iron keeps three peaks in HFD of $\mathrm{FeCr}$ (2.36 at.\%) foil spectrum at $T=293 \mathrm{~K}$, influencing only on a ratio of their intensities (Figure 42) whereas in a case of nanoparticles a very small peak with $H_{e} \approx 31.75 \mathrm{~T}$ is 


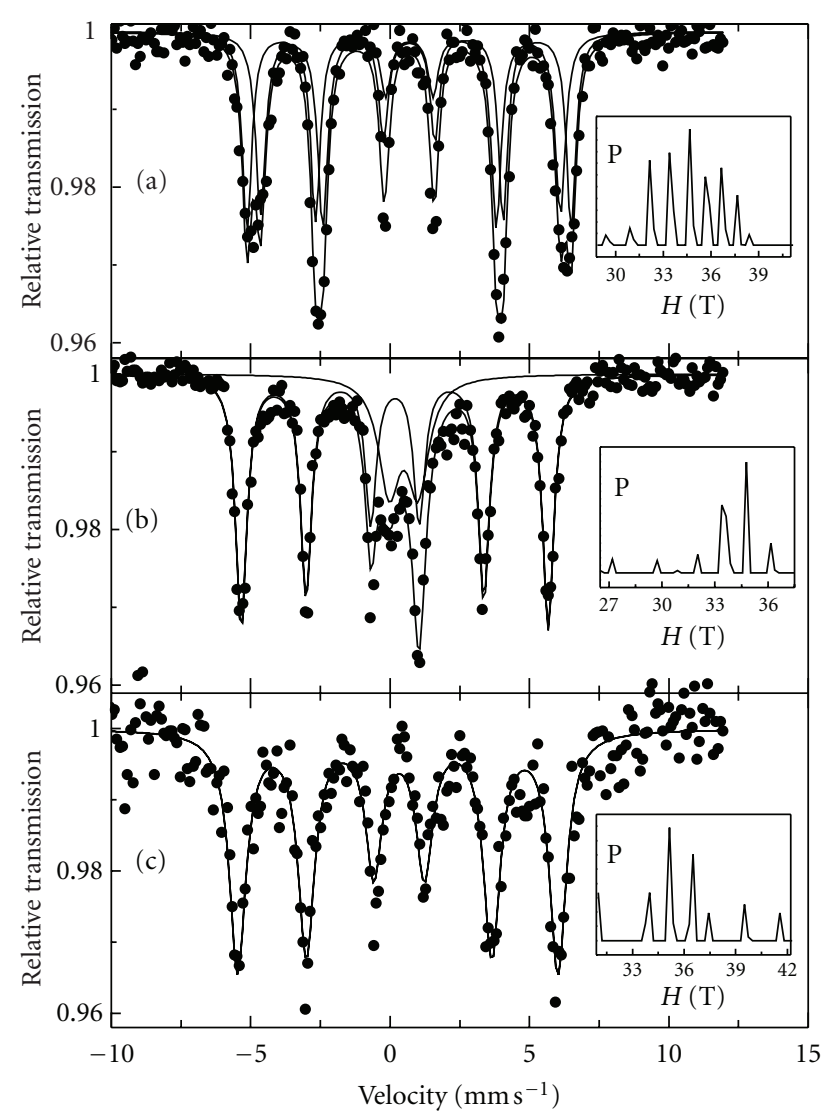

Figure 40: Mössbauer spectra for FePd (49.6\%). Hyperfine field distribution for a ferromagnetic component in spectrum is shown in inserts. (a) the bulk alloy, $T=293 \mathrm{~K}$; (b) particles with a size of $D=12 \pm 3 \mathrm{~nm}$ at $T=293 \mathrm{~K}$; (c) the same particles at $T=100 \mathrm{~K}$

added to the basic peak $\left(H_{e} \approx 32.9 \mathrm{~T}\right)$ (Figure $\left.42(\mathrm{~b})\right)$ [150]. Cooling of $\mathrm{FeCr}$ (2.36 at.\%) particles to $80 \mathrm{~K}$ is accompanied by disappearance of the former and occurrence of a poorly expressed peak $\left(H_{e} \approx 34.8 \mathrm{~T}\right)$ at the right side of the basic peak $\left(H_{e}=34.4 \mathrm{~T}\right)$. An arising simultaneously sextet related to $\mathrm{Fe}$ oxide turns out to be much weaker than that at pure iron (Figure 41(c)). Hence, chrome presence interferes developing Fe oxide on $\mathrm{FeCr}$ (2.36 at.\%) particles. As a result, magnetic interaction of the particle core with oxide shell becomes weaker. It is just the reason why, after cooling of the sample to $80 \mathrm{~K}$ and its subsequent warming to room temperature, a magnitude of the hyperfine field corresponding to unique peak in HFD for $\mathrm{FeCr}$ (2.36 at.\%) particles remains practically invariable (Figure $42(\mathrm{~d})$ ).

A little bit different results show FeCo particles with a size of $D \approx 13 \mathrm{~nm}[79,147]$. In HFD for FeCo $(10$ at.\%) and FeCo (50 at.\%) alloys there are three main peaks whereas at FeCo (85 at.\%) alloy near a point of bcc $\rightarrow$ fcc transition the number of peaks increases, and they are divided on two groups (Figure 43). Cobalt makes appreciable impact on $3 d-$ electrons of $\mathrm{Fe}$ atoms, giving rise to a discontinuity of HFD because it is a good ferromagnetic.

So, as a result of alloying of iron with $\mathrm{Pd}, \mathrm{Cr}$, and $\mathrm{Co}$, a set of peaks arises in HFD. However, as follows from Figure 41,

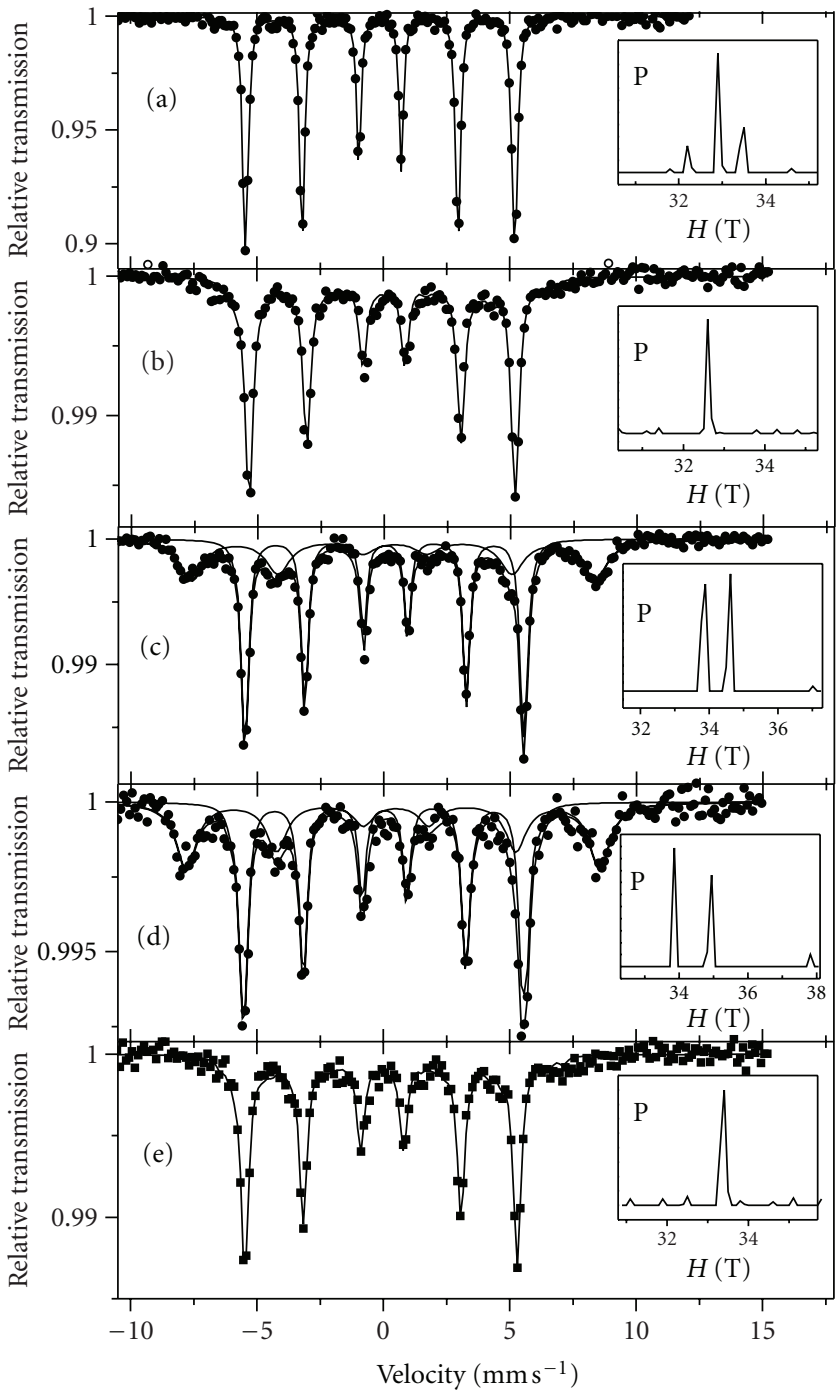

FIGURE 41: Mössbauer spectra for pure Fe foil (a) and nanoparticles ((b)-(e)) of $D=13 \pm 2 \mathrm{~nm}$ size at different temperatures. (a) $293 \mathrm{~K}$; (b) $293 \mathrm{~K}$; (c) $80 \mathrm{~K}$; (d) $4.2 \mathrm{~K}$; (e) particles preliminarily aged at $80 \mathrm{~K}$ and subsequently warmed up to $293 \mathrm{~K}$.

HFD for a pure Fe foil also contains three peaks though in this case action of additives is absent. As above mentioned, the massive pure iron of a different origin, as a rule, had three main peaks in HFD, and Fe nanoparticles with a size of $D \approx 13-15 \mathrm{~nm}$ showed only one peak. Even at increasing the size of Fe particles to $D=117 \mathrm{~nm}$ this unique peak remained and did not change the value (Figure 44) [145]. On the other hand, as seen in Figure 44, for BM iron when regions of X-ray coherency attain a size of several tens of nanometers in grains of about $1 \mu \mathrm{m}$ size, their HFD show several peaks, evidencing a significant role of the magnetic block structure when forming a discontinuity of HFD. Small single domain particles, weakly interacting with each other, are characterized by a presence of the unique peak in HFD. A close contact of the single domain coherency areas in grains at ball milling leads to their strong magnetic interaction and discontinuity of HFD. The similar pattern takes place in the 


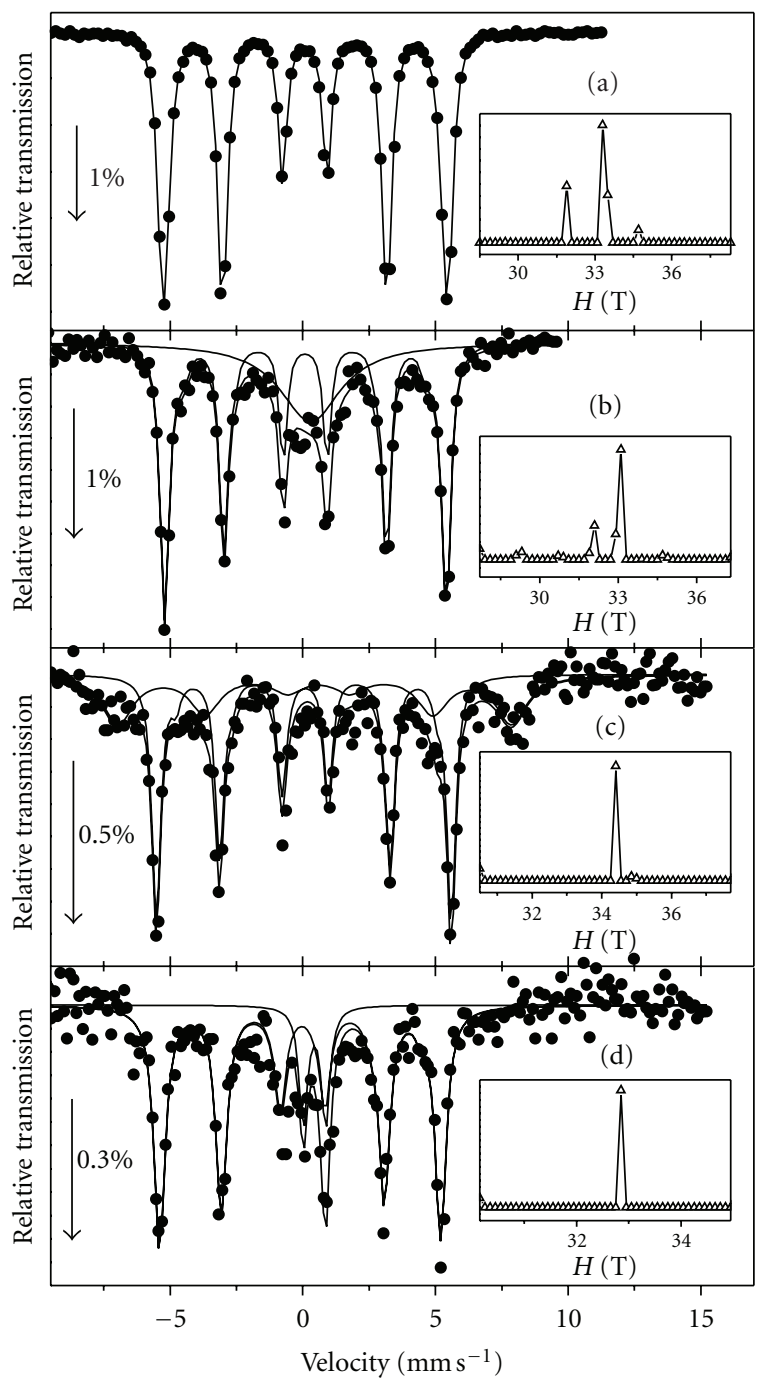

Figure 42: Mössbauer spectra for a bulk FeCr (2.36 at.\%) alloy (a) and as-fresh nanoparticles of the same composition ((b)-(d)) of $D=13 \pm 2 \mathrm{~nm}$ size at different temperatures. (a) A thin foil, $293 \mathrm{~K}$; (b) $293 \mathrm{~K}$; (c) $80 \mathrm{~K}$; (d) particles preliminarily aged at $80 \mathrm{~K}$ and subsequently warmed up to $293 \mathrm{~K}$.

iron foil magnetic domains which also interact with each other. When along a foil plane the external magnetic field of $0.03 \mathrm{~T}$ is applied which is sufficient to align the magnetic moments of domains parallel to the field, then thereby the multidomain structure of the foil turns to a single domain. As a result, instead of three initial peaks HFD shows only a unique peak, as at small single domain particles (Figure 45) [145].

An example of close contact single domain bcc and fcc regions is "abnormal" Fe particles with a size of $50 \mathrm{~nm}$ prepared in the levitation-flow generator by Guen and Miller $[21,22]$. Mössbauer spectra of abnormal Fe particles have shown the availability of two hyperfine fields with the average values $H_{i} \approx 33.0$ and $36.6 \mathrm{~T}$ (Figure 3 ) [43, 44]. The higher hyperfine field $H_{e} \approx 36.6 \mathrm{~T}$ was referred to

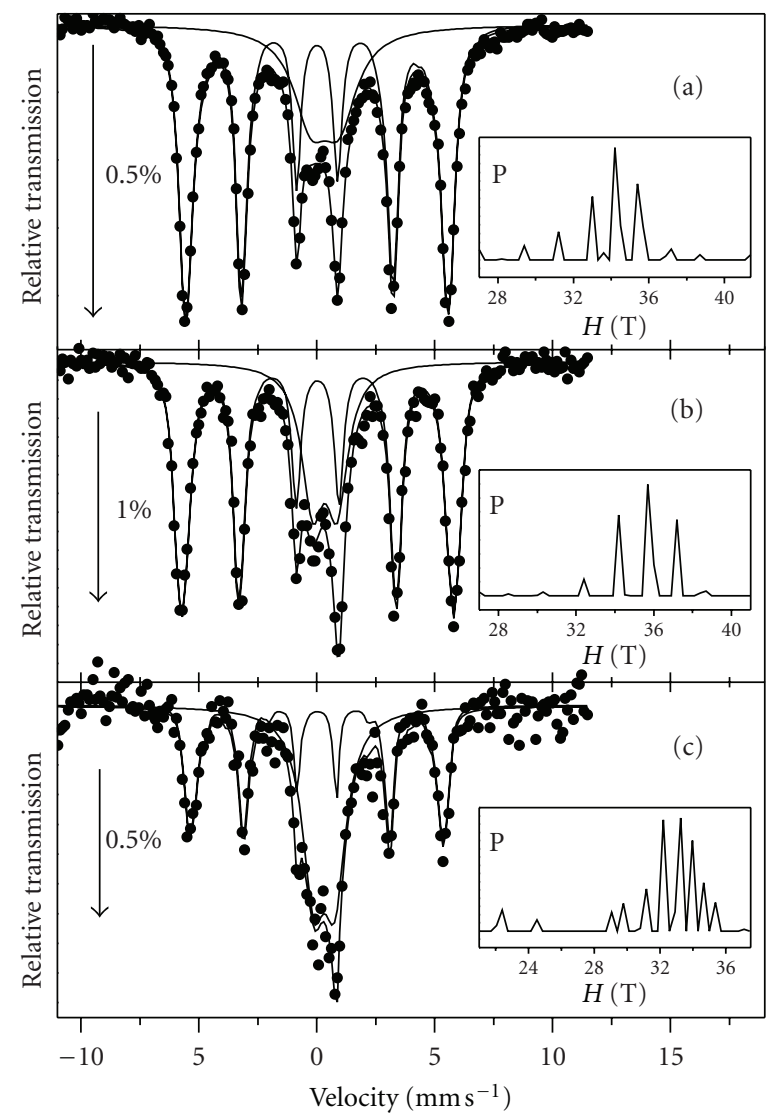

FIgUre 43: Mössbauer spectra of FeCo particles with a size of $D \approx$ $15 \mathrm{~nm}$ containing (a) 10, (b) 50, and (c) 85 Co at.\%.

regions of bcc phase kept the magnetic order of the hightemperature fcc phase. As seen in Figure 46(a), HFD for abnormal Fe particles consists of two groups of peaks [144]. With decreasing temperature down to $80 \mathrm{~K}$ a number of peaks in every group grows by unity (Figure 46(b)). If one compares the spectrum in Figure 8 with that in Figure 46(a) taken more than 10 years later, then it is easy seen some narrowing of lines and a change of two sextets areas ratio so that the fraction of the magnetic order specific to bcc phase rises at the expense of decrease of the contribution from the bcc regions having a magnetic order of the hightemperature fcc phase. This distinction is likely due to ageing of the powder. At $4.2 \mathrm{~K}$ spectrum (Figure $46(\mathrm{c})$ ) it is seen only one peak belonged to bcc Fe whereas the second group expands to seven nearly equidistant peaks. In the external magnetic field of $6 \mathrm{~T}$ all spins of abnormal particles tend to be oriented along it; therefore, lines 2 and 5 disappear (Figure 47). At the same time, the average hyperfine fields go from $H_{e}=33.8 \mathrm{~T}$ and $H_{e}=37.1 \mathrm{~T}(4.2 \mathrm{~K})$ down to $H_{e}=$ $29.5 \mathrm{~T}$ and $H_{e}=33.5 \mathrm{~T}(10 \mathrm{~K}, 6 \mathrm{~T})$; that is, they decrease by 4.3 and $3.6 \mathrm{~T}$, respectively. This indicates that the hyperfine fields are opposite to the external field and slightly inclined to it. One can distinctly see in its spectrum (Figure 47(a)) that two components with the average fields $H_{e} \approx 33.0$ and 36.2 $\mathrm{T}$ dominate in HFD given in the inset. 


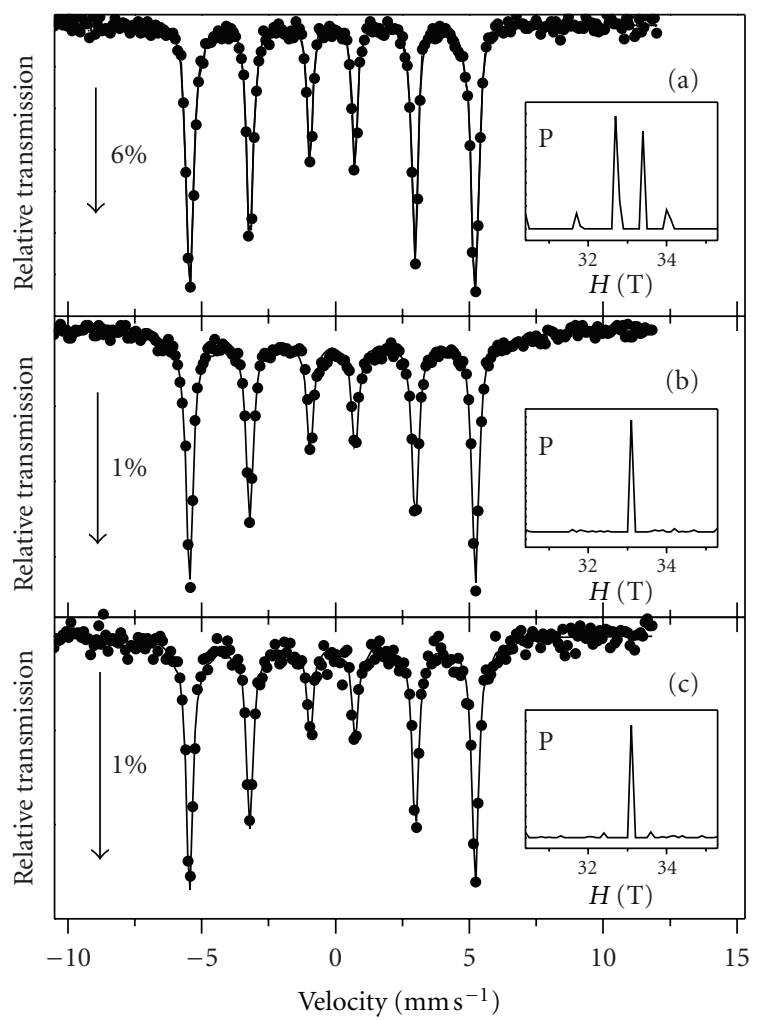

FIGURE 44: Mössbauer spectra of BM Fe powder (a) and Fe particles with a size of 20 (b) and 117 (c) nm.

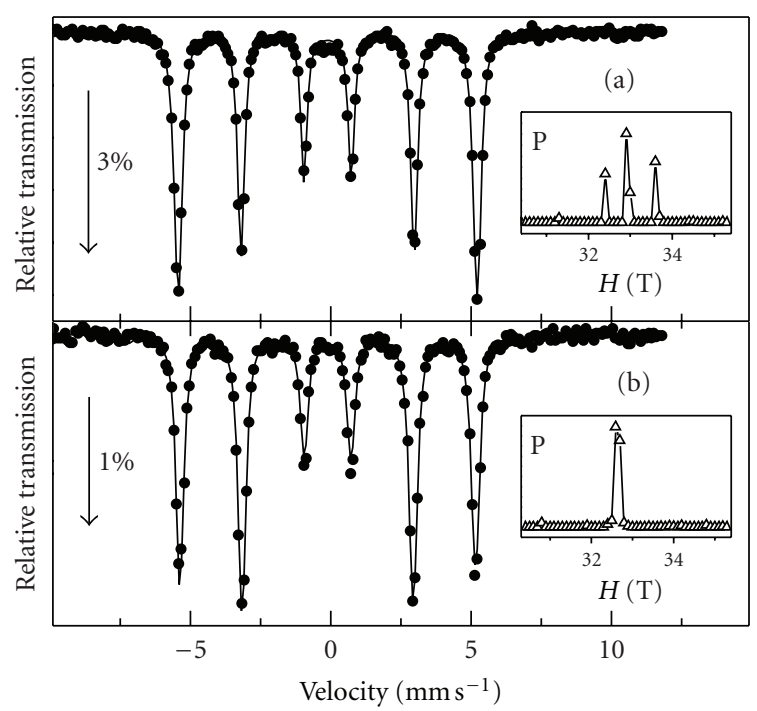

Figure 45: Influence of the external magnetic field of $0.03 \mathrm{~T}$ on the HFD shape for Fe foil. (a) An external magnetic field is lacking; (b) in an applied magnetic field.

5.3. Testing the NORMOS Program. Testing the NORMOS code to separate the adjacent closely spaced hyperfine fields has been carried out for different thin Fe foil applied for the set-up calibration [145]. Probation of the code operation was carried out by four ways: (1) effect of a smoothing parameter $\lambda$ on the HFD shape with its changing from zero to $0.1 ;$ (2)

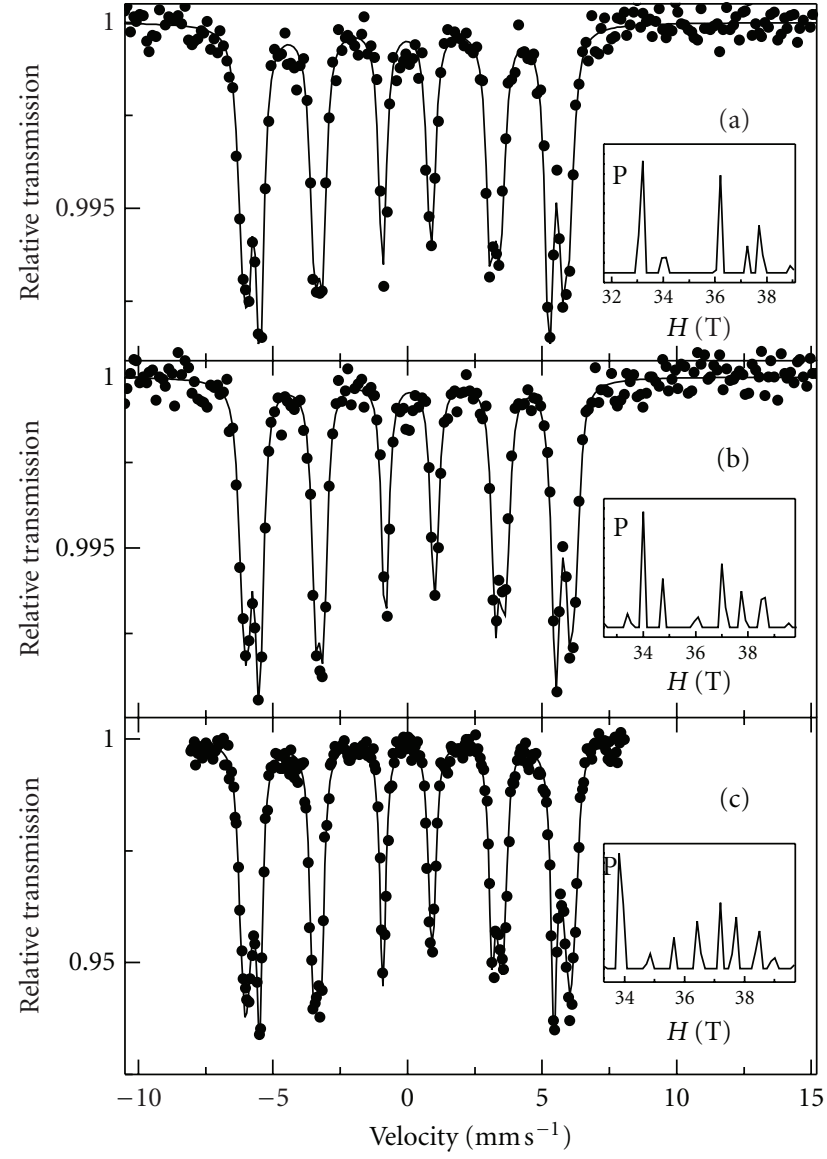

FIGURE 46: Mössbauer spectra of abnormal aerosol Fe particles with a size of $D=50 \mathrm{~nm}$ at different temperatures. (a) $293 \mathrm{~K}$; (b) $80 \mathrm{~K}$; (c) $4.2 \mathrm{~K}$.

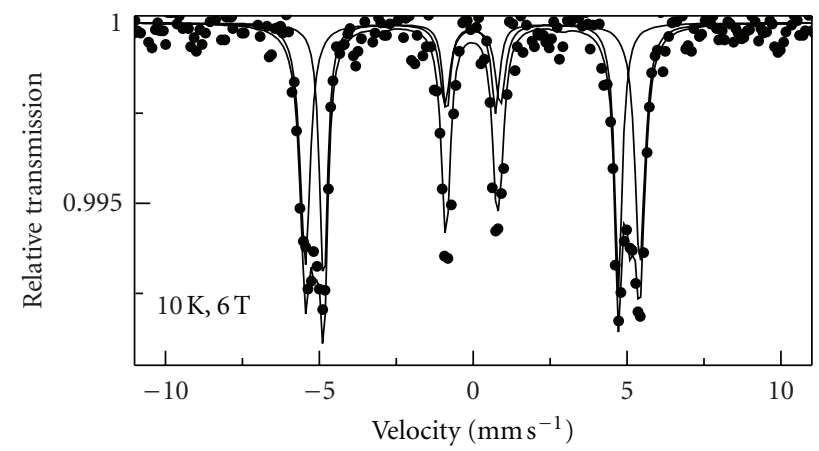

Figure 47: In-field Mössbauer spectrum of abnormal aerosol $\mathrm{Fe}$ particles at $T=10 \mathrm{~K}$ and $H=6 \mathrm{~T}$.

variation of a step for change of hyperfine field between the adjacent trial sextets $(\Delta)$ within $0.05-0.5 \mathrm{~T}$; (3) change of the hyperfine field value for the first trial sextet $\left(H_{\mathrm{oi}}\right)$ from 30.5 to $31.55 \mathrm{~T}$; (4) change of the trial sextet linewidth (l) from 0.1 to $0.25 \mathrm{~mm} \times \mathrm{s}^{-1}$. As has been noted, HFD for the Fe foils examined shows three main completely separated peaks. The location of these peaks did not change when varying parameters $H_{\mathrm{oi}}$ and $\Delta$ within the above-given range. 
With increase $\Delta$ of 0.05 to $0.5 \mathrm{~T}$ three peaks are gradually broadened and finally merged each other. Thus, only a choice of $\lambda=0$ permitted to reveal a discrete structure in HFD.

The similar testing was carried out also for small $(\sim$ $13 \mathrm{~nm}$ ) aerosol Fe particles when parameters $H_{\mathrm{oi}}, \Delta$, and $\lambda$ were varied [143]. The results exhibited that a quite broad sampling of these parameters as in the case of iron foils did not practically change HFD pattern. Mössbauer spectra showed always only one peak though their sextet linewidths were somewhat larger than in the case of $\mathrm{Fe}$ foils. Invariance of the peak location has been found out when changing $H_{\mathrm{oi}}$ from $20.1 \mathrm{~T}$ to $32.20 \mathrm{~T}$. Changing a step $\Delta$ of trial sextets from 0.01 to $0.5 \mathrm{~T}$ has no influence on a peak location and gives rise to an apparent broadening (Figure 48). This is due to the large value of trial sextet linewidth $\left(l=0.34-0.36 \mathrm{~mm} \times \mathrm{s}^{-1}\right)$ selected by the program.

To get an idea concerning influence of variable parameters on two closely spaced peaks in HFD the given procedure has been applied to the low temperature Mössbauer spectra of Fe nanoparticles (Figures 41(c) and 41(d)). HFD extracted from the spectra taken at 80 and $4.2 \mathrm{~K}$ has shown two individual intense peaks. The location of the peaks $\left(H_{i}=33.9\right.$ and $34.9 \mathrm{~T}$ ) was kept invariable with decrease of $H_{\mathrm{oi}}$ in the range of 25.3 to $30.08 \mathrm{~T}$. Note that a space between two peaks remains equal about $1 \mathrm{~T}$. At the same time a number of trial sextets within this range ( $1 \mathrm{~T}$ ) diminish with increase $\Delta$ from $0.05 \mathrm{~T}$ to $0.8 \mathrm{~T}$. At $\Delta=0.8 \mathrm{~T}$ both peaks fall on two adjacent trial sextets and look like as if they would merge with each other. Hence, there is a loss of information when two actual hyperfine fields cease to be distinguished (Figure 49).

The influence of the smoothing parameter $\lambda$ on a shape of HFD for the $80 \mathrm{~K}$ Mössbauer spectrum of small Fe nanoparticles is shown in Figure 50. It is seen that two peaks are clearly separated at values $\lambda$ of zero to $10^{-3}$ whereas at $\lambda=10^{-2}$ the peaks merged with each other. In all cases the high fitting goodness has been attained (see captions to Figure 50), but two peaks spaced by $1 \mathrm{~T}$ appear to be well separated only at $\lambda=0$.

Basing on the results obtained for Fe foils and nanoparticles, one may conclude that the increase of step $\Delta$ between trial sextets to about $0.5-0.8 \mathrm{~T}$ and smoothing parameter $\lambda$ to 0.1 results in a complete smearing of detailed structure in HFD at ${ }^{57} \mathrm{Fe}$ nuclei. Note that the suggested technique to elicit a fine structure of HFD is based not only on broadening lines in some Mössbauer spectrum but also on taking into account a characteristic shape of the lines. This as well as the possibility to detect quite weak $(<1 \mathrm{~T})$ magnetic interactions in a solid body is unquestionable advantages of the given technique.

5.4. On the Origin of Discontinuity of the Hyperfine Fields at ${ }^{57} \mathrm{Fe} \mathrm{Nuclei}$. It is easily seen at the spectra for massive $\mathrm{Fe}$ and its alloys that the peaks in HFD are grouped nearby the averaged magnitudes for the hyperfine field at Fe nuclei in pure iron, with some of them being spaced on each side of the ones. Appearance in the HFD for the various Fe foils of the peak with a higher field than that inherent to a singledomain state of iron $\left(H_{i} \approx 33 \mathrm{~T}\right)$ rouses the most interest.
This could be by no means due to any change of the electron properties of atoms at different sites in the sample because the ferromagnetic impurities are lacking or negligible, and vacancies close to the resonating Fe atoms could only reduce the hyperfine field strength. Moreover, the peak in the HFD referred to the higher field strength by about $0.5 \mathrm{~T}$ than that for the main peak $\left(H_{i} \approx 33 \mathrm{~T}\right)$ disappears when the foil is magnetized almost to saturation.

One reason for the HFD discreteness may be a sample to be multidomain [151]. Indeed, there are three main peaks in the HFD for a multidomain Fe foil, while there is only one peak at the single-domain small particles or in case of the same Fe foil magnetized up to about its saturation, when it is close to a single-domain state. Formation of multidomain structure in ferromagnetics is caused with its lower energy compared with the energy of the saturation homogenously magnetized sample. Each domain binds a number of the nearest collinear spins by exchange interactions that is equivalent to action of the effective molecular Weiss' field attaining a magnitude of order $10^{3} \mathrm{~T}$ [152]. This field defines the temperature-dependent magnetization of sample [151] though it does not affect on an HFD pattern. Dipole interaction of domains is weaker by magnitude of several orders, but it is just that leads to the appearance of an additional internal magnetic field in the sample. The magnetic moments of the nearest-neighbor domains usually form closed loops so that, in the mean, the overall sample is demagnetized.

Meanwhile, the closely contacted domains in closed loops might have the opposite magnetic moments. They interact with each other and so the effective internal magnetic fields arise in adjacent domains. As a result, a slight Zeeman splitting of energy levels for mainly d-electrons of Fe atoms may occur. The latter, in turn, changes polarization of selectrons close to $\mathrm{Fe}$ nuclei. In this connection, in addition to the main peak with $H_{i} \approx 33 \mathrm{~T}$, there appear two less intensity peaks corresponding the higher and lower hyperfine fields than that for the main peak. Such pattern is observed for demagnetized Fe foils and ball-milled Fe powder with grains having coherency regions of about $10 \mathrm{~nm}$ [132]. Since these regions are very close each other, they are able to interact. Therefore, HFD for BM Fe powder shows several peaks (Figure 44(a)) like for the Fe foil (Figure 45(a)).

By applying the external magnetic field, the domains with the opposite magnetic moments disappear, eliminating the possibility to generate the effective internal field in the adjacent domains. In this case the discontinuity in HFD might not arise that is evidenced by one peak in the HFD for the in-field Fe foil Mössbauer spectrum given in Figure 45(b).

A single peak in HFD is observed for an ensemble of disordered single-domain Fe nanoparticles which are quite far from each other. According to many magnetic measurements a critical size for single-domain Fe particle is $20-25 \mathrm{~nm}$ [1]. Therefore merely Fe nanoparticles of 13 and $20 \mathrm{~nm}$ size are totally single-domain whereas the larger $\mathrm{Fe}$ nanoparticles $(30,38,76$, and $117 \mathrm{~nm})$ may consist of two and more domains. However, due to thermal fluctuations of the magnetic moments of these domains at room temperature, 


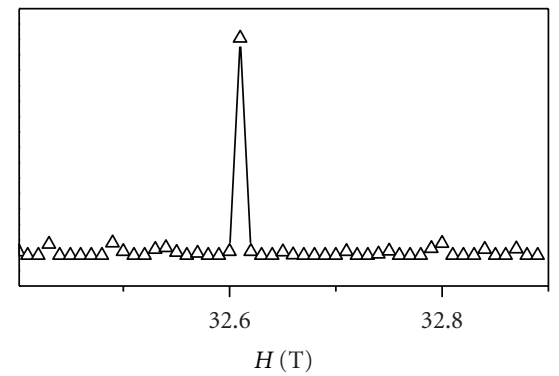

(a)

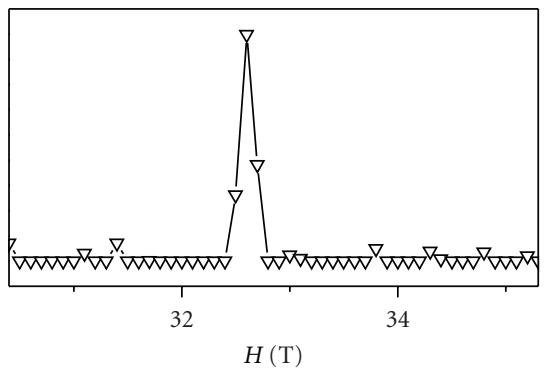

(b)

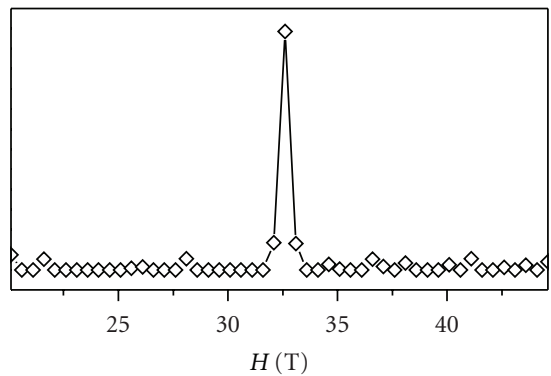

(c)

FIGURE 48: Effect of value $\triangle$ on HFD shape extracted from room temperature Mössbauer spectrum for small Fe particles. (a) $\triangle=0.01 \mathrm{~T}$ $\left(\lambda=0, l=0.36 \mathrm{~mm} \cdot \mathrm{s}^{-1}, \mathrm{HR}=1.612, \chi^{2}=2.066\right) ;(\mathrm{b}) \Delta=0.1 \mathrm{~T}\left(\lambda=0, l=0.35 \mathrm{~mm} \cdot \mathrm{s}^{-1}, \mathrm{HR}=1.873, \chi^{2}=2.401\right) ;(\mathrm{c}) \Delta=0.5 \mathrm{~T}(\lambda=0, l=$ $\left.0.34 \mathrm{~mm} \cdot \mathrm{s}^{-1}, \mathrm{HR}=1.707, \chi^{2}=2.188\right)$.

their interaction is weak and not ever able to create the closed loops and a sufficient internal local magnetic field leading to appearance of several peaks in the HFD. Figure 44(c) confirms this supposition. With decreasing the temperature fluctuations of the magnetic moments go down and the interaction of domains grows. Nevertheless, in the case of small single-domain nanoparticles their interaction is apparently insufficient to promote occurrence of closed magnetic loops and additional peaks in the HFD even at a temperature as low as $4.2 \mathrm{~K}$ (Figure $41(\mathrm{~d})$ ).

Any additives to iron having impact on the electron states of its $3 d$-shell should give rise to discreteness of hyperfine field at ${ }^{57} \mathrm{Fe}$ nuclei. Pd is easy polarized under $\mathrm{Fe}$ atoms in particles [153] that likely results in quantization of $3 d-$ electrons at $\mathrm{Fe}$ atoms. In turn, this changes somewhat a contact Fermi interaction $[154,155]$.

\section{Conclusive Remarks}

Synthesis, structure, and magnetic properties of aerosol particles of iron and its alloys have been considered. The nanoparticles were prepared by the gas evaporation technique having a set of distinctions. The apparent advantage is a possibility to produce near spherical particles of the reference mean dimension from about 2 to $10^{3} \mathrm{~nm}$ with a narrow size distribution. Extremely rapid cooling rate during preparation enables one to freeze high-temperature states and to reveal the effect of high-temperature Fe modification on magnetic properties of particles of pure iron and its alloys. Effect of Fe oxides, content of which may attain 30-40\%, on the particles shell has been thoroughly examined.

A particular attention has been paid to the analysis of the hyperfine fields at ${ }^{57} \mathrm{Fe}$ nuclei. The substantiation to apply the NORMOS code for separating closely located hyperfine fields has been considered. For iron and its some alloys both as a bulk and aerosol nanoparticles it was shown that a hyperfine field distribution has a discrete character with peaks spaced by $0.5-1.0 \mathrm{~T}$. The existence of several peaks in HFD for bulk Fe has been supposed to be caused with the internal magnetic fields owing to its multidomain structure. This argument has been corroborated by transformation of the HFD pattern for Fe bulk after applying the external magnetic field of $0.03 \mathrm{~T}$. At 


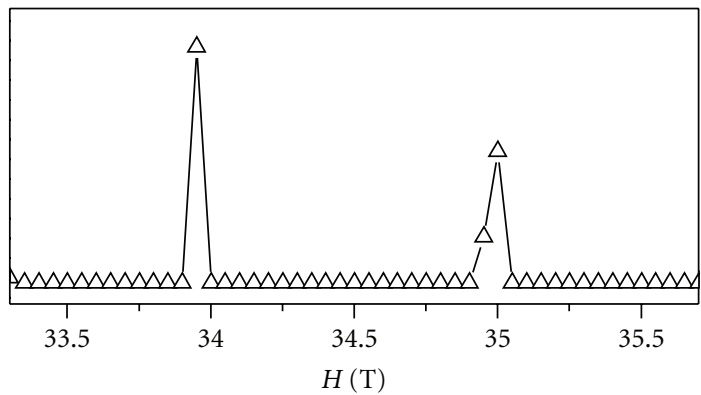

(a)

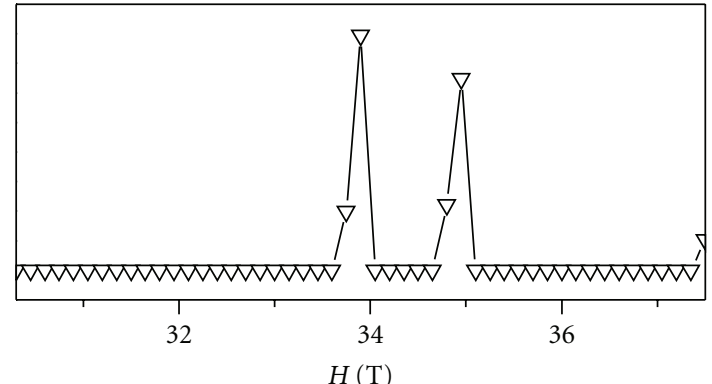

(b)

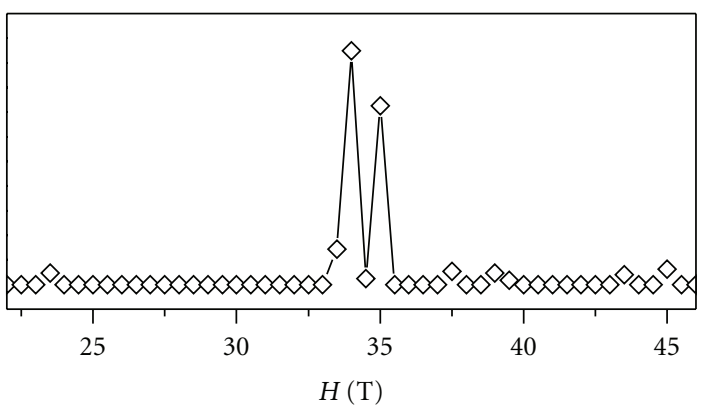

(c)

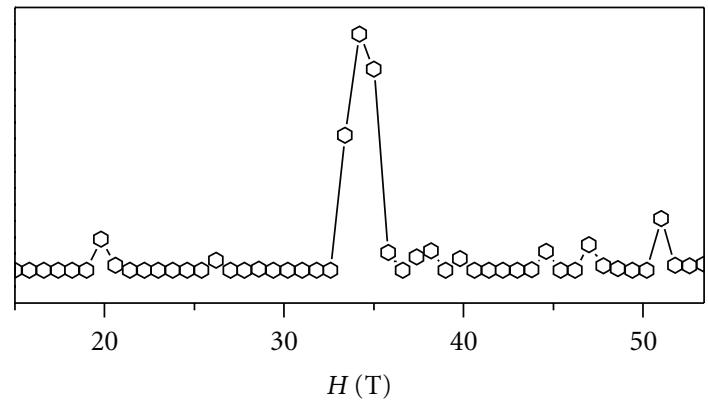

(d)

Figure 49: Effect of value $\triangle$ on HFD shape extracted from $4.2 \mathrm{~K}$ Mössbauer spectrum for small Fe particles. (a) $0.05 \mathrm{~T}\left(\lambda=0, H_{\mathrm{oi}}=33.3 \mathrm{~T}, l\right.$ $\left.=0.36 \mathrm{~mm} \cdot \mathrm{s}^{-1} ; \mathrm{HR}=0.8414, \chi^{2}=1.099\right) ;(\mathrm{b}) \Delta=0.15 \mathrm{~T}\left(\lambda=0, H_{\mathrm{oi}}=30.3 \mathrm{~T}, l=0.34 \mathrm{~mm} \cdot \mathrm{s}^{-1} ; \mathrm{HR}=0.8204, \chi^{2}=1.071\right) ;(\mathrm{c}) \Delta=0.5 \mathrm{~T}(\lambda=0$, $\left.H_{\mathrm{oi}}=22.0 \mathrm{~T}, l=0.33 \mathrm{~mm} \cdot \mathrm{s}^{-1} ; \mathrm{HR}=0.7795, \chi^{2}=1.012\right) ;(\mathrm{d}) \Delta=0.8 \mathrm{~T}\left(\lambda=0, H_{\mathrm{oi}}=15.0 \mathrm{~T}, l=0.31 \mathrm{~mm} \cdot \mathrm{s}^{-1} ; \mathrm{HR}=0.7593, \chi^{2}=0.9917\right)$. 

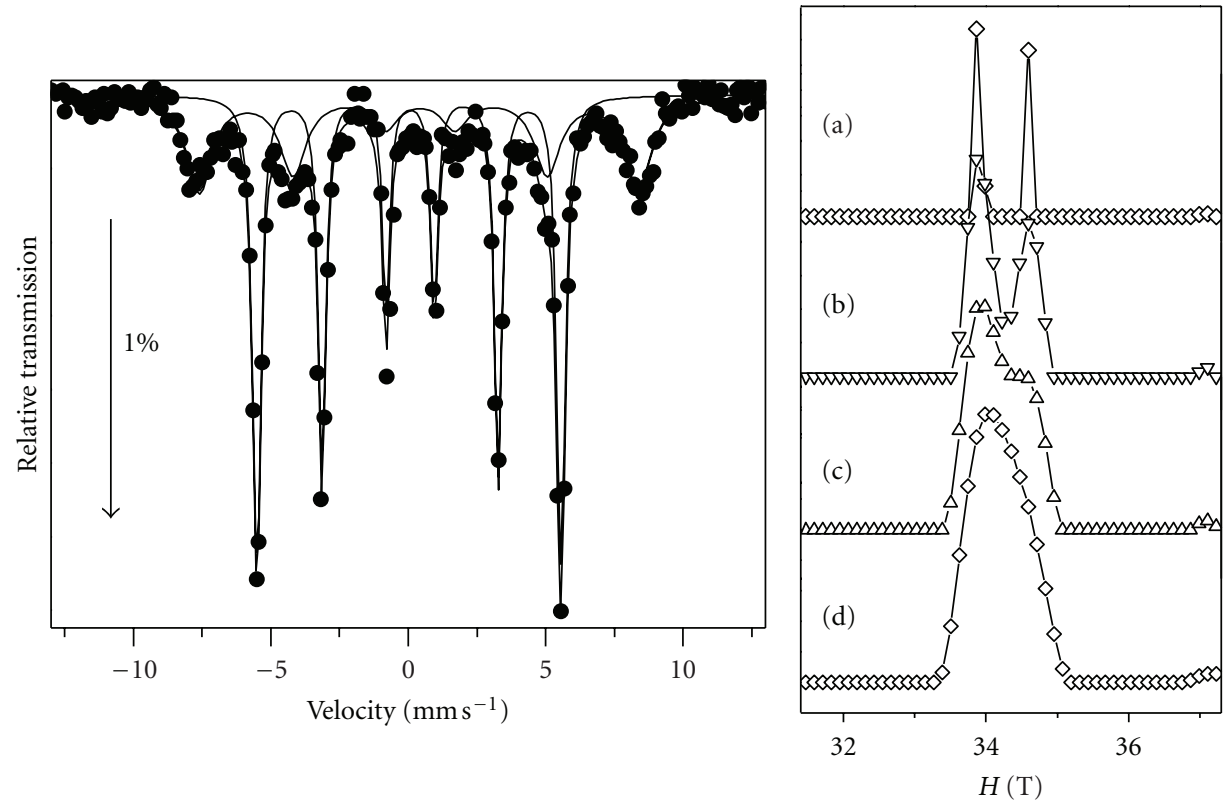

FiguRE 50: Smoothing parameter $\lambda$ dependent HFD (right) extracted from $80 \mathrm{~K}$ Mössbauer spectrum (left) for small Fe particles $\left(H_{\mathrm{oi}}=\right.$ $\left.31.47 \mathrm{~T}, l=0.31 \mathrm{~mm} \cdot \mathrm{s}^{-1}, \Delta=0.15 \mathrm{~T}\right)$. (a) $\lambda=0\left(\mathrm{HR}=2.085, \chi^{2}=2.727\right)$; (b) $\lambda=10^{-3}\left(\mathrm{HR}=2.088,\left(\chi^{2}=2.731\right)\right.$; (c) $\lambda=10^{-2}(\mathrm{HR}=2.092$, $\left.\chi^{2}=2.736\right) ;(\mathrm{d}) \lambda=0.05\left(\mathrm{HR}=2.095, \chi^{2}=2.740\right)$.

the same time, aerosol Fe nanoparticles being single-domain show only a unique peak in HFD.

The revealed phenomenon of HFD discontinuity is evidently of fundamental significance because it enables to detect very weak magnetic interactions which are inaccessible to other techniques.

\section{Acknowledgments}

The work is partially supported by the Russian Foundation for Basic Research (Grant 05-03-32068a). Some Mössbauer measurements, including low temperature ones, have been fulfilled in Institut de Ciència de Materials de Barcelona (ICMAB-CSIC), and one of the authors (E. A. Shafranovsky) is grateful to E. Molins and A. Roig for the opportunity to make them and to L. Casas and M. Gich for the assistance. Authors wish to express their gratitude to G. Principi of Padova University for taking low temperature Mössbauer spectra of FeNi and FeMn nanoparticles and to J. M. Greneche of Université du Maine (France) for carrying out in-field Mössbauer measurements of abnormal $50 \mathrm{~nm} \mathrm{Fe}$ particles. The foils of FeNi, FeMn, FeNiMn, and FePt alloys were provided by V. A. Makarov of TsNIIChermet.

\section{References}

[1] Yu. I. Petrov, Fizika Malykh Chastits (Physics of Small Particles), Nauka, Moscow, Russia, 1982.

[2] Yu. I. Petrov, Klastery i Malye Chastitsy (Clusters and Small Particles), Nauka, Moscow, Russia, 1986.

[3] Yu. I. Petrov, "Preparation, some properties, and applications of aerosol particles of metals, their alloys. and compounds," Khimicheskaya fizika, vol. 22, no. 3, pp. 3-14, 2003 (Russian).
[4] M. Ratner and D. Ratner, Nanotechnology, Pearson Education, Prentice Hall, Upper Saddle River, NJ, USA, 2003.

[5] G. Xiao, J. Q. Wang, and P. Xiong, "Giant magnetoresistance and anomalous Hall effect in $\mathrm{Co}-\mathrm{Ag}$ and $\mathrm{Fe}-\mathrm{Cu}, \mathrm{Ag}, \mathrm{Au}, \mathrm{Pt}$ granular alloys," IEEE Transactions on Magnetics, vol. 29, no. 6, pp. 2694-2699, 1993.

[6] C. Peng and D. Dai, "Magnetic properties and magnetoresistance in granular Fe-Cu alloys," Journal of Applied Physics, vol. 76, no. 5, pp. 2986-2990, 1994.

[7] J. Tang, "Magnetic properties of a few material systems made by mechanical alloying and milling," Materials Science Forum, vol. 235-238, pp. 819-824, 1997.

[8] U. K. Rössler, B. Idzikowski, D. Eckert et al., "Structural, magnetic and magnetotransport properties of melt-spun $\mathrm{Fe}_{10} \mathrm{Cu}_{90}$," IEEE Transactions on Magnetics, vol. 35, no. 5, pp. 2841-2843, 1999.

[9] Q. A. Pankhurst and R. J. Pollard, "Fine-particle magnetic oxides," Journal of Physics, vol. 5, no. 45, pp. 8487-8508, 1993.

[10] Q. A. Pankhurst, J. Connolly, S. K. Jones, and J. Dobson, "Applications of magnetic nanoparticles in biomedicine," Journal of Physics D, vol. 36, no. 13, pp. R167-R181, 2003.

[11] Q. A. Pankhurst, N. K. T. Thanh, S. K. Jones, and J. Dobson, "Progress in applications of magnetic nanoparticles in biomedicine," Journal of Physics D, vol. 42, no. 22, Article ID 224001, 15 pages, 2009.

[12] P. Gorria, D. Martinez-Blanco, J. A. Blanco et al., "Invar effect in fcc-FeCu solid solutions," Physical Review B, vol. 69, no. 21, Article ID 214421, 5 pages, 2004.

[13] Yu. I. Petrov, "Nanoscale materials for microelectronics. New technology," in Proceedings of the 1st International Conference on Progress in Inorganic and Organometallic Chemistry, $\mathrm{P}$. Zdroj, Poland, F. P. Pruchnik, and M. Zuber, Eds., pp. 228239, Wroclaw University, 1994.

[14] M. Y. Guen, E. A. Velichenkova, I. V. Eryomina, and M. S. Ziskin, "On the conditions of formation and properties 
of $\mathrm{Ag}-\mathrm{Cu}$ alloy in a finely divided form," Fizika Tverdogo Tela (Soviet Physics, Solid State), vol. 6, pp. 1622-1626, 1964 (Russian).

[15] G. A. Lontsova and Yu. I. Petrov, "Highly dispersed films of cadmium selenide," Doklady Akademii Nauk SSSR, vol. 303, pp. 1407-1410, 1988 (Russian).

[16] M. Y. Guen, M. S. Ziskin, and Yu. I. Petrov, "A study of the dispersion of aluminium aerosols as dependent on the conditions of their formation," Doklady Akademii Nauk SSSR, vol. 127, no. 2, pp. 366-368, 1959 (Russian).

[17] Yu. I. Petrov and E. A. Shafranovsky, "Features of ultrafine inorganic particle preparation by the gas evaporation method," Bulletin of the Russian Academy of Sciences, vol. 64, no. 8, pp. 1236-1244, 2000.

[18] E. A. Shafranovsky and Yu. I. Petrov, "Aerosol nanoparticles of metals, alloys and compounds: synthesis, properties and potential applications," in Aerosols: Properties, Sources and Management Practices, Ya. Alekseyev and K. Plisetskaya, Eds., Nova Science, New York, NY, USA, 2012.

[19] M. Y. Guen and Yu. I. Petrov, "Disperse condensates of metal vapour," Russian Chemical Reviews, vol. 38, no. 12, pp. 10071024, 1969.

[20] E. A. Shafranovsky and Yu. I. Petrov, "Aerosol Fe nanoparticles with the passivating oxide shell," Journal of Nanoparticle Research, vol. 6, no. 1, pp. 71-90, 2004.

[21] M. Y. Guen and A. V. Miller, "Levitation method for producing ultrafine powders of metals," Surface-Physics, Chemistry, Mechanics, no. 2, pp. 150-154, 1983 (Russian).

[22] M. Y. Guen and A. V. Miller, "Method for producing aerosols of metals by evaporating a levitated and RF field heated drop of metal," Byulleten Izobretiniia, no. 11, p. 251981 (Russian), USSR Inventor's Certificate no. 814432.

[23] A. N. Jigach, I. O. Leipunskiy, M. L. Kuskov, N. I. Stoenko, and V. B. Storozhev, "An apparatus for the production and study of metal nanoparticles," Instruments and Experimental Techniques, vol. 43, no. 6, pp. 839-845, 2000.

[24] Yu. I. Petrov, "High-temperature X-ray chamber for studying powders," Pribory i Tekhnika Eksperimenta, no. 4, pp. 162164, 1963 (Russian).

[25] L. Lutterrotti and S. Gialanella, "X-ray diffraction characterization of heavily deformed metallic specimens," Acta Materialia, vol. 46, no. 1, pp. 101-110, 1997.

[26] R. A. Brand, "Improving the validity of hyperfine field distributions from magnetic alloys," Nuclear Instruments and Methods in Physics Research Section B, vol. 28, pp. 398-416, 1987.

[27] E. C. Stoner and E. P. Wohlfarth, "A mechanism of magnetic hysteresis in heterogeneous alloys," Philosophical Transactions of the Royal Society A, vol. 240, no. 826, pp. 599-642, 1948.

[28] T. Ibusuki, S. Kojima, O. Kitakami, and Y. Shimada, "Magnetic anisotropy and behaviors of Fe nanoparticles," IEEE Transactions on Magnetics, vol. 37, no. 4, pp. 2223-2225, 2001.

[29] I. S. Jacobs and C. P. Bean, "An approach to elongated fineparticle magnets," Physical Review, vol. 100, no. 4, pp. 10601067, 1955.

[30] R. H. Kodama, A. E. Berkowitz, E. J. McNiff Jr., and S. Foner, "Surface spin disorder in $\mathrm{NiFe}_{2} \mathrm{O}_{4}$ nanoparticles," Physical Review Letters, vol. 77, no. 2, pp. 394-397, 1996.

[31] R. H. Kodama and A. E. Berkowitz, "Atomic-scale magnetic modeling of oxide nanoparticles," Physical Review B, vol. 59, no. 9, pp. 6321-6336, 1999.
[32] R. H. Kodama, "Magnetic nanoparticles," Journal of Magnetism and Magnetic Materials, vol. 200, no. 1-3, pp. 359372, 1999.

[33] Yu. I. Petrov, "Surface pressure in clusters and small particles-is it real?” Phase Transitions, vol. 24-26, no. 2, pp. 407-417, 1990.

[34] Yu. I. Petrov, "A new view on the surface stresses in condensed media," Phase Transitions, vol. 45, no. 4, pp. 221-250, 1993.

[35] Yu. I. Petrov, "On the surface stress and surface pressure in clusters and small particles," in Progress in the Physics of Clusters, G. N. Chuev, V. D. Lachno, and A. P. Nefedov, Eds., pp. 431-457, World Scientific, Singapore, 1999.

[36] W. F. Schlosser, "A model for the Invar alloys and the Fe-Ni system," Journal of Physics and Chemistry of Solids, vol. 32, no. 5, pp. 939-949, 1971.

[37] B. Window, "Invar anomalies," Journal of Applied Physics, vol. 44, no. 6, pp. 2853-2861, 1973.

[38] E. F. Wassermann, "New developments on the invar-effect," Physica Scripta, vol. T25, pp. 209-219, 1989.

[39] E. F. Wassermann and P. Entel, "Invar, AF-Invar, anti-Invar and martensite in fcc Fe-based alloys: an attempt to order this chaos," Journal de Physique (4), vol. 5, pp. C8-287-C8-292.

[40] L. Kaufman, E. V. Clougherty, and R. J. Weiss, "The lattice stability of metals-III. Iron,” Acta Metallurgica, vol. 11, no. 5, pp. 323-335, 1963.

[41] R. J. Weiss, "The origin of the "invar" effect," Proceedings of the Physical Society, vol. 82, no. 2, pp. 281-288, 1963.

[42] R. J. Weiss, “The invar effect," Philosophical Magazine, vol. 26, pp. 261-263, 1972.

[43] Yu. I. Petrov and E. A. Shafranovsky, "Exhibition of highand low-spin states of the high-temperature fcc phase in nanoparticles of Fe, Fe-rich and Co-rich alloys," Journal of Nanoparticle Research, vol. 3, no. 5-6, pp. 419-432, 2001.

[44] Y. V. Baldokhin and Yu. I. Petrov, "Two states of the facecentered-cubic structure in iron discovered while investigating the Mössbauer spectra and the thermal expansion of small particles," Soviet physics, Doklady, vol. 37, pp. 563-565, 1992.

[45] H. Esser and H. Eusterbrock, "Untersuchung der Wärmeausdehnung von einigen Metallen and Legierungen mit einem verbesserten Dilatometer," Archiv fuer das Eisenhuettenwes, vol. 14, pp. 341-355, 1941.

[46] Z. S. Basinski, W. Hume-Rothery, and A. L. Sutton, "The lattice expansion of iron," Proceedings of the Royal Society A, vol. 229, no. 1179, pp. 459-467, 1955.

[47] M. Acet, H. Zayres, E. F. Wassermann, and W. Pepperhoff, "High-temperature moment-volume instability and antiInvar of $\gamma$-Fe," Physical Review B, vol. 49, no. 9, pp. 60126017, 1994.

[48] Y. V. Baldokhin, P. Y. Kolotyrkin, Yu. I. Petrov, and E. A. Shafranovsky, "Structure and Mössbauer spectra of ultrasmall particles of iron-enriched Fe-Mn alloys," Doklady Physics, vol. 40, pp. 491-494, 1995.

[49] Y. V. Baldokhin, P. Y. Kolotyrkin, Yu. I. Petrov, and E. A. Shafranovsky, "Structural and magnetic transformations in Fe-rich fine Fe-Mn particles," Physics Letters A, vol. 211, no. 4, pp. 237-241, 1996.

[50] Y. V. Baldokhin, P. Y. Kolotyrkin, Yu. I. Petrov, and E. A. Shafranovsky, "Comparative study of the structure and local magnetic order in bulk and ultrafine particles of Fe-Mn (32\%-35\%) and $\mathrm{Fe}_{3} \mathrm{Pt}$," Journal of Applied Physics, vol. 82, no. 6, pp. 3042-3046, 1997. 
[51] Yu. I. Petrov, E. A. Shafranovsky, Y. V. Baldokhin, and G. A. Kochetov, "On ferro- and antiferromagnetic ordering in ultrafine particles of Fe-rich $\mathrm{Fe}-\mathrm{Ni}$ and $\mathrm{Fe}-\mathrm{Mn}$ alloys," Journal of Applied Physics, vol. 86, no. 12, pp. 7001-7005, 1999.

[52] Y. V. Baldokhin, P. Y. Kolotyrkin, I. I. Morozov, Yu. I. Petrov, and E. A. Shafranovsky, "Structural transformations of small $\mathrm{Fe}-\mathrm{Ni}$ particles with a change in the hyperfine parameters of the Mössbauer spectra," Physics-Doklady, vol. 38, pp. 226228, 1993.

[53] Y. V. Baldokhin, P. Y. Kolotyrkin, Yu. I. Petrov, and E. A. Shafranovsky, "Some specific features of fine Fe and Fe-Ni particles," Journal of Applied Physics, vol. 76, no. 10, pp. 64966498, 1994.

[54] Y. V. Baldokhin, P. Y. Kolotyrkin, Yu. I. Petrov, and E. A. Shafranovsky, "On the exhibition of high and low spin states of the fcc phase in ultrafine $\mathrm{Fe}$ and Fe-Ni particles," Physics Letters A, vol. 189, no. 1-2, pp. 137-139, 1994.

[55] Y. V. Baldokhin, P. Y. Kolotyrkin, Yu. I. Petrov, and E. A. Shafranovsky, "Comparison of the structure of the Mössbauer spectra of ultrasmall particles and bulk alloy $\mathrm{Fe}_{3}$ Pt," Doklady Physical Chemistry, vol. 347, pp. 45-48, 1996.

[56] V. Papaefthymiou, A. Kostikas, A. Simopoulos et al., "Magnetic hysteresis and Mössbauer studies in ultrafine iron particles," Journal of Applied Physics, vol. 67, no. 9, pp. 44874489, 1990.

[57] Y. Endoch and Y. Ishikawa, "Antiferromagnetism of $\gamma$ iron manganese alloys," Journal of the Physical Society of Japan, vol. 30, pp. 1614-1627, 1971.

[58] O. G. Sokolov and A. I. Mel'ker, "Invar effect in ironmanganese alloys," Doklady Akademii Nauk SSSR, vol. 159, pp. 74-76, 1964 (Russian).

[59] R. Bauminger, S. G. Cohen, A. Marinov, S. Ofer, and E. Segal, "Study of the low-temperature transition in magnetite and the internal fields acting on iron nuclei in some spinel ferrites, using Mössbauer absorption,” Physical Review, vol. 122, no. 5, pp. 1447-1450, 1961.

[60] Y. V. Baldokhin, Yu. I. Petrov, and E. A. Shafranovsky, "Structural and magnetic transformations of Fe-rich Fe-NiMn alloy under transition from bulk solid to fine particles," Bulletin of the Russian Academy of Sciences, vol. 62, pp. 919925, 1998.

[61] Yu. I. Petrov, E. A. Shafranovsky, and Y. V. Baldokhin, "Structure and local magnetic order in ultrafine particles of $\mathrm{Fe}_{65}\left(\mathrm{Ni}_{1-x} \mathrm{Mn}_{x}\right)_{35}(0 \leq x \leq 1)$ alloys," Solid State Communications, vol. 113, no. 3, pp. 153-158, 2000.

[62] M. Shiga, "Magnetic properties of $\mathrm{Fe}_{65}\left(\mathrm{Ni}_{1-x} \mathrm{Mn}_{x}\right)_{35}$ ternary alloys," Journal of the Physical Society of Japan, vol. 22, no. 2, pp. 539-546, 1967.

[63] B. Huck, F. Saurenbach, and J. Hesse, "Investigation of spin structure in fcc $\mathrm{Fe}-\mathrm{Ni}-\mathrm{Mn}$," Hyperfine Interactions, vol. 28, no. 1-4, pp. 479-482, 1986.

[64] M. Fricke and J. Hesse, "Mössbauer study indicating magnetic clusters in FeNiMn reentrant spin glass alloys," Hyperfine Interactions, vol. 70, pp. 1105-1108, 1992.

[65] E. A. Owen, E. L. Yates, and A. H. Sully, "An X-ray investigation of pure iron-nickel alloys. Part 4: the variation of lattice-parameter with composition," Proceedings of the Royal Society of London, vol. 49, pp. 315-322, 1937.

[66] M. Shiga, "Influence of magnetization on lattice constants of 3D transition metal alloys," Solid State Communications, vol. 10, no. 12, pp. 1233-1236, 1972.
[67] A. E. Vol, Sructure and Properties of Binary Metal System, Fizmatgiz, Moscow, Russia, 1962.

[68] O. Kubaschewsky, Iron-Binary Phase Diagrams, Springer, Berlin, Germany, 1982.

[69] E. A. Owen, E. L. Yales, and A. H. Sully, "An X-ray investigation of pure iron-nickel alloys. Part 5: the variation of thermal expansion with composition," Proceedings of the Royal Society of London, vol. 49, pp. 323-325, 1937.

[70] Y. V. Baldokhin, P. Y. Kolotyrkin, V. A. Makarov, Yu. I. Petrov, and E. A. Shafranovsky, "Phase transitions, crystallographic and local magnetic ordering of $\mathrm{Fe}_{3} \mathrm{Pt}$," Phase Transitions, vol. 64, no. 4, pp. 239-247, 1998.

[71] Yu. I. Petrov and E. A. Shafranovsky, "Correlation of the structure and Mössbauer spectra of ultrafine particles and bulk Fe-Co alloy as functions of the composition," Doklady Physics, vol. 44, pp. 605-609, 1999.

[72] P. J. Murray and J. W. Linnit, "Mössbauer studies in the spinel system $\mathrm{Co}_{x} \mathrm{Fe}_{3-x} \mathrm{O}_{4}$," Journal of Physics and Chemistry of Solids, vol. 37, no. 6, pp. 619-624, 1976.

[73] C. E. Johnson, M. S. Ridout, and T. E. Cranshaw, "The Mössbauer effect in iron alloys," Proceedings of the Physical Society, vol. 81, no. 6, pp. 1079-1090, 1963.

[74] B. De Mayo, D. W. Forster, and S. Spooner, "Effects of atomic configurational changes on hyperfine interactions in concentrated iron-cobalt alloys," Journal of Applied Physics, vol. 41, pp. 1319-1320, 1970.

[75] I. Vincze, I. A. Campbell, and A. J. Meyer, "Hyperfine field and magnetic moments in b.c.c. Fe-Co and Fe-Ni," Solid State Communications, vol. 15, no. 9, pp. 1495-1499, 1974.

[76] E. Jartych, J. K. Zurawicz, and M. Budzynski, "A Mössbauer study of electrodeposited $\mathrm{Fe}_{1-x} \mathrm{Co}_{x}$ alloys," Journal of Physics, vol. 5, no. 7, pp. 927-934, 1993.

[77] Y. V. Baldokhin, V. V. Cherdyntsev, S. D. Kaloshkin, and I. A. Tomilin, "Hyperfine structure of iron-cobalt-system alloys prepared by mechanical fusion," Doklady Physics, vol. 43, no. 7, pp. 397-399, 1998.

[78] Y. Muraoka, T. Fujiwara, M. Shiga, and Y. Nakamura, "Sharp transition from ferromagnetic to antiferromagnetic state in fcc Fe-Co particles precipitated in Cu matrix," Journal of the Physical Society of Japan, vol. 50, no. 10, pp. 3284-3291, 1981.

[79] Yu. I. Petrov and E. A. Shafranovsky, "Specific features of the hyperfine field at iron nuclei in aerosol nanoparticles of FeCo alloy," Doklady Physical Chemistry, vol. 440, no. 1, pp. 178182, 2011.

[80] Yu. I. Petrov, "Structure and thermal expansion of small Co particles," Kristallografiya, vol. 11, pp. 931-932, 1966 (Russian).

[81] J. Slater, The Self-Consistent Field for Molecules and Solids, McGrow-Hill, New York, NY, USA, 1974.

[82] F. Seitz, The Modern Theory of Solids, McGraw-Hill, New York, NY, USA, 1940.

[83] Yu. I. Petrov, E. A. Shafranovsky, Y. F. Krupyanskii, and S. V. Esin, "Specific features of the structure and local magnetic order in nanoparticles of the Fe-Cr alloy," Doklady Physical Chemistry, vol. 379, no. 1-3, pp. 194-198, 2001.

[84] Yu. I. Petrov, E. A. Shafranovsky, Y. F. Krupyanskii, and S. V. Essine, "Structure and Mössbauer spectra for the Fe-Cr system: from bulk alloy to nanoparticles," Journal of Applied Physics, vol. 91, no. 1, pp. 352-361, 2002.

[85] E. A. Shafranovsky, Yu. I. Petrov, M. Gich et al., "Structural and magnetic properties of bulk alloys and aerosol nanoparticles in the $\mathrm{Fe}_{100-x} \mathrm{Cr}_{x}$ system," Journal of Alloys and Compounds, vol. 416, no. 1-2, pp. 51-57, 2006. 
[86] B. G. Bergman and D. P. Shoemaker, "The determination of the crystal structure of the phase in the iron-chromium and iron-molybdenum systems," Acta Crystallographica, vol. 7, pp. 857-865, 1954.

[87] G. J. Dickins, A. M. B. Douglas, and W. H. Taylor, "Sigmaphase in the Co-Cr and Fe-Cr systems," The Journal of the Iron and Steel Institute, vol. 167, 27 pages, 1951.

[88] G. D. Preston, "X-ray examination of chromium-iron alloys," The Journal of the Iron and Steel Institute, vol. 124, pp. 139$141,1931$.

[89] G. D. Preston, "XXXV. An X-ray examination of ironchromium alloys," Philosophical Magazine, vol. 13, no. 84, pp. 419-425, 1932.

[90] A. P. Gulyaev and E. F. Trusova, "Regularities of changes in the properties of solid solutions," Zhurnal Tekhnicheskoi Fiziki, vol. 20, pp. 66-78, 1950 (Russian).

[91] A. L. Sutton and W. Hume-Rothery, “. The lattice spacings of solid solutions of titanium, vanadium, chromium, manganese, cobalt and nickel in $\alpha$-iron," Philosophical Magazine, vol. 46, no. 383, pp. 1295-1309, 1955.

[92] T. De Nys and P. M. Gielen, "Spinodal decomposition in the Fe-Cr system," Metallurgical Transactions, vol. 2, no. 5, pp. 1423-1428, 1971.

[93] H. Yamomoto, "A study on the nature of aging of Fe-Cr alloys by means of the Mössbauer effect," Japanese Journal of Applied Physics, vol. 3, pp. 745-748, 1964.

[94] M. Shiga and Y. Nakamura, "Effect of local environment on formation of local moments in bcc Fe-Cr alloys-Mössbauer study," Journal of the Physical Society of Japan, vol. 49, no. 2, pp. 528-534, 1980.

[95] P. A. Beck, "Magnetic behavior of intermediate phases in alloys of transition elements," Transactions. American Institute of Mining, Metallurgical and Petroleum Engineers, vol. 194, p. 420, 1952.

[96] S. M. Dubiel and J. Zukrowski, "Mössbauer effect study of charge and spin transfer in Fe-Cr," Journal of Magnetism and Magnetic Materials, vol. 23, no. 2, pp. 214-228, 1981.

[97] H. Kuwano and K. Ono, "Mössbauer study of $\mathrm{Fe}_{1-x} \mathrm{Cr}_{x}$ alloys," Journal of the Physical Society of Japan, vol. 42, pp. 72-75, 1977.

[98] Y. Li, W. Gong, G. C. Hadjipanayis et al., "Size effects on the magnetic properties of fine $\mathrm{Fe}-\mathrm{Cr}$ particles," Journal of Magnetism and Magnetic Materials, vol. 130, no. 1-3, pp. 261-266, 1994.

[99] M. V. Nevitt and A. T. Aldred, "Ferromagnetism in V-Fe and Cr-Fe alloys," Journal of Applied Physics, vol. 34, no. 3, pp. 463-468, 1963.

[100] W. Gong, H. Le, Z. Zhao et al., "Magnetic properties of $\mathrm{Fe}-\mathrm{Cr}$ ultrafine particles," Journal of Applied Physics, vol. 70, no. 10, pp. 5900-5902, 1991.

[101] A. Tasaki, M. Takao, and H. Tokunaga, "Magnetic properties of ferromagnetic metal alloy fine particles prepared by evaporation in inert gasses. II," Japanese Journal of Applied Physics, vol. 13, pp. 271-276, 1974.

[102] P. A. Flinn and S. L. Ruby, "Local magnetic fields in $\mathrm{Fe}-\mathrm{Al}$ alloys," Physical Review, vol. 124, no. 1, pp. 34-36, 1961.

[103] K. Wertheim, V. Jaccardino, J. H. Wernick, and D. N. E. Buchanan, "Range of the exchange interaction in iron alloys," Physical Review Letters, vol. 12, no. 1, pp. 24-27, 1964.

[104] B. Fultz, "Chemical systematics of iron-57 hyperfine magnetic field distributions in iron alloys," in Mössbauer Spectroscopy Applied to Magnetism and Material Science, G. J. Long and F. Grandjean, Eds., vol. 1, Plenum Press, New York, NY, USA, 1993.
[105] A. T. Aldred, B. D. Rrainford, J. S. Kouvel, and T. J. Hicks, "Ferromagnetism in iron-chromium alloys. II. Neutron scattering studies," Physical Review B, vol. 14, no. 1, pp. 228234, 1976.

[106] E. A. Shafranovsky, Yu. I. Petrov, L. Casas, and E. Molin, "Structural and Mössbauer studies of aerosol FeCu nanoparticles in a wide composition range," Journal of Nanoparticle Research, vol. 13, pp. 4913-4928, 2011.

[107] J. Z. Jiang, C. Gente, and R. Bormann, "Mechanical alloying in the $\mathrm{Fe}-\mathrm{Cu}$ system," Materials Science and Engineering A, vol. 242, no. 1-2, pp. 268-277, 1998.

[108] C. L. Chien, S. H. Liou, D. Kofalt et al., "Magnetic properties of $\mathrm{Fe}_{x} \mathrm{Cu}_{100-x}$ solid solutions," Physical Review B, vol. 33, no. 5, pp. 3247-3250, 1986.

[109] W. Keune, J. Lauer, and D. L. Williamson, "Mössbauer studies of FeCu films," Journal of Physics, vol. 35, pp. C6-473-C6-478, 1974.

[110] J. Klein, T. Heck, S. J. Campbell, F. Aubertin, and U. Gonser, "The activation energy for $\gamma$-Fe precipitates in CuFe," Hyperfine Interactions, vol. 54, no. 1-4, pp. 811-816, 1990.

[111] B. Window, "Hyperfine fields at ${ }^{57} \mathrm{Fe}$ in copper iron alloys," Journal of Physics C, vol. 3, no. 3, pp. S323-S329, 1970.

[112] J. Z. Jiang, U. Gonser, C. Gente, and R. Borman, "Thermal stability of the unstable fcc- $\mathrm{Fe}_{50} \mathrm{Cu}_{50}$ phase prepared by mechanical alloying," Applied Physics Letters, vol. 63, no. 8, pp. 1056-1058, 1993.

[113] A. Hernando, P. Crespo, A. R. Yavari, A. Garcia-Escorial, and J. M. Barandiaran, "Formation of $\gamma$-Fe by mechanical alloying of $\mathrm{Cu}-\mathrm{Fe}$ ?" IEEE Transactions on Magnetics, vol. 29, no. 6, pp. 2634-2636, 1993.

[114] P. Crespo, A. Hernando, R. Yavari et al., "Magnetic behavior of metastable fcc Fe-Cu after thermal treatments," Physical Review B, vol. 48, no. 10, pp. 7134-7139, 1993.

[115] P. Crespo, A. Hernando, A. G. Escorial, K. M. Kemner, and V. G. Harris, "Extended X-ray-absorption fine-structure studies of heat-treated fcc- $\mathrm{Fe}_{50} \mathrm{Cu}_{50}$ powders processed via highenergy ball milling," Journal of Applied Physics, vol. 76, no. 10, pp. 6322-6324, 1994.

[116] A. Hernando, P. Crespo, A. Garsia-Escorial, and J. M. Barandiaran, "Comment on 'mechanically driven alloying of immiscible elements," Physical Review Letters, vol. 70, no. 22, article 3521, 1993.

[117] Y. Yang, Y. Zhu, Q. S. Li et al., "Mechanical alloying, fine structure and thermal decomposition of nanocrystalline FCC- $\mathrm{Fe}_{60} \mathrm{Cu}_{40}$," Physica B, vol. 293, no. 3-4, pp. 249-259, 2001.

[118] E. A. Shafranovsky, Yu. I. Petrov, L. Casas, and E. Molins, "Structure and composition of aerosol particles obtained on evaporation of a homogeneous $\mathrm{FeCu}(50.4$ at\%) alloy," Doklady Physical Chemistry, vol. 429, no. 2, pp. 246-251, 2009.

[119] K. Sumiyama, Y. Nakamura, and K. Tanaka, "Mössbauer spectra and electronic structure of nonequilibrium $\mathrm{Fe}-\mathrm{Cu}$ alloys produced by vapor quenching," Hyperfine Interactions, vol. 53, no. $1-4$, pp. 143-158, 1990.

[120] Q. A. Pankhurst, M. F. Thomas, C. E. Johnson, R. Zquiak, X. $\mathrm{X}$. Zhang, and J. Tejada, "Mössbauer study of Fe $(5 \AA)+\mathrm{Cu}$ (50 ̊) multilayers," IEEE Transactions on Magnetics, vol. 30, no. 2, pp. 778-780, 1994.

[121] S. C. Abrahams, L. Guttman, and J. S. Kasper, "Neutron diffraction determination of antiferromagnetism in facecentered cubic $(\gamma)$ iron," Physical Review, vol. 127, no. 6, pp. 2052-2055, 1962. 
[122] U. Gonser, C. J. Meechan, A. H. Muir, and H. Wiedersich, "Determination of néel temperatures in fcc iron," Journal of Applied Physics, vol. 34, no. 8, pp. 2373-2378, 1963.

[123] G. J. Johanson, M. B. McGirr, and D. A. Wheeler, "Determination of the néel temperature of face-centered-cubic iron," Physical Review B, vol. 1, no. 7, article 3208, 1970.

[124] W. Keune, R. Halbauer, U. Gonser, J. Lauer, and D. L. Williamson, "Antiferromagnetism of fcc Fe thin films," Journal of Applied Physics, vol. 48, no. 7, pp. 2976-2979, 1977.

[125] R. Halbauer and U. Gonser, "Antiferromagnetism of fcc iron films," Journal of Magnetism and Magnetic Materials, vol. 35, no. 1-3, pp. 55-56, 1983.

[126] W. A. A. Macedo and W. Keune, "Magnetism of epitaxial fcc$\mathrm{Fe}(100)$ films on $\mathrm{Cu}(100)$ investigated in situ by conversionelectron Mössbauer spectroscopy in ultrahigh vacuum," Physical Review Letters, vol. 61, no. 4, pp. 475-478, 1988.

[127] W. Keune, T. Ezawa, W. A. A. Macedo, U. Glos, K. P. Schletz, and U. Kirschbaum, "Magneto-volume effects in $\gamma$ Fe ultrathin films and small particles," Physica B, vol. 161, no. 1-3, pp. 269-275, 1989.

[128] J. L. Tholence and R. Tournier, "One-impurity and interaction effects on the $\mathrm{Cu}$ : Fe magnetization," Physcial Review Letters, vol. 25, no. 13, pp. 867-871, 1970.

[129] U. Gradmann and H. O. Isbert, "Ferromagnetic $\gamma$-iron films prepared on $\mathrm{CuAu}(111)$-surfaces," Journal of Magnetism and Magnetic Materials, vol. 15-18, no. 2, pp. 1109-1111, 1980.

[130] M. Donath, M. Pickel, A. B. Schmidt, and M. Weinelt, "Ferromagnetic Fe on $\mathrm{Cu}(001)$ throughout the fcc-like phase: arguing from the viewpoint of the electronic structure," Journal of Physics, vol. 21, no. 13, Article ID 134004, 6 pages, 2009.

[131] A. Roig, X. X. Zhang, R. Zuberek, J. Tejada, and E. Molins, "Magnetic properties of $\mathrm{Fe} \mathrm{Cu}$ multilayers," Journal of Magnetism and Magnetic Materials, vol. 140-144, no. 1, pp. 559-560, 1995.

[132] L. Del Bianco, C. Ballesteros, J. M. Rojo, and A. Hernando, "Magnetically ordered fcc structure at the relaxed grain boundaries of pure nanocrystalline Fe," Physical Review Letters, vol. 81, no. 20, pp. 4500-4503, 1998.

[133] P. Gorria, D. Martinez-Blanco, J. A. Blanco et al., "Hightemperature induced ferromagnetism on $\gamma$-Fe precipitates in FeCu solid solutions," Physical Review B, vol. 72, no. 1, Article ID 014401, 7 pages, 2005.

[134] A. Yousif, K. Bouziane, M. E. Elzain et al., "Magnetic properties of nanocrystalline $\mathrm{Fe}_{x} \mathrm{Cu}_{1-x}$ alloys prepared by ball milling," Hyperfine Interactions, vol. 156-157, no. 1-4, pp. 213-221, 2004.

[135] P. Chubing, C. Haiying, L. Guozhong, and D. Daosheng, "Magnetic properties of $\mathrm{Fe}_{x} \mathrm{Cu}_{1-x}$ granular alloy films," Journal of Applied Physics, vol. 76, no. 10, pp. 7102-7104, 1994.

[136] N. H. Duc, N. A. Tuan, A. Fnidiki et al., "Structural, magnetic and Mössbauer studies of $\mathrm{Fe}-\mathrm{Cu}$ granular films," Journal of Physics Condensed Matter, vol. 14, no. 26, pp. 6657-6666, 2002.

[137] H. Q. Guo, X. D. Ma, L. Y. Yang, B. G. Shen, and J. G. Zhao, "Structure, magnetic properties and stability of the interfaces in $\mathrm{Fe} / \mathrm{Cu}$ multilayer," Journal of Magnetism and Magnetic Materials, vol. 99, no. 1-3, pp. 199-203, 1991.

[138] P. J. Schurer, B. Scully, M. Kowalewski, and B. Heinrich, "Mössbauer investigation of the $\mathrm{Fe} / \mathrm{Cu}$ interfaces in BCC $\mathrm{Fe} / \mathrm{Cu} / \mathrm{Fe}(001)$ structures," Journal of Magnetism and Magnetic Materials, vol. 224, no. 1, pp. 65-75, 2001.
[139] B. Bandyopadhyay, B. Pahari, and K. Ghoshray, "Magnetization and ${ }^{63} \mathrm{Cu}$ NMR studies on granular FeCu alloys," Physical Review B, vol. 76, no. 21, Article ID 214424, 6 pages, 2007.

[140] V. P. Parfenova, N. N. Delyagin, A. L. Erzinkyan, and S. I. Reyman, "Local spin configurations of Fe atoms in the $\mathrm{Rh}_{1-x} \mathrm{Fe}_{x}(x=0.1,0.2$, and 0.3$)$ system with competing exchange interactions," Physics of the Solid State, vol. 42, no. 8, pp. 1465-1470, 2000.

[141] A. L. Erzinkyan, N. N. Delyagin, V. P. Parfenova, and S. I. Reyman, "Origin of the strong influence of rhodium on the Curie temperature of $\mathrm{Pd}-\mathrm{Fe}$ alloys: the spin reorientation in $\left(\mathrm{Pd}_{100-x} \mathrm{Rh}_{x}\right)_{90} \mathrm{Fe}_{10}$ alloys," Journal of Magnetism and Magnetic Materials, vol. 231, no. 1, pp. 20-22, 2001.

[142] N. N. Delyagin, A. L. Erzinkyan, V. P. Parfenova, and S. I. Reyman, "Antiferromagnetic spin correlations in palladiumbased $\mathrm{Pd}-\mathrm{Fe}, \mathrm{Pd}-\mathrm{Fe}-\mathrm{Ag}$, and $\mathrm{Pd}-\mathrm{Fe}-\mathrm{Rh}$ magnetic alloys," Journal of Experimental and Theoretical Physics, vol. 95, no. 6, pp. 1056-1061, 2002.

[143] Yu. I. Petrov and E. A. Shafranovsky, "On the conditions eliciting a detailed structure in the hyperfine field distribution at ${ }^{57} \mathrm{Fe}$ nuclei," Nuclear Instruments and Methods in Physics Research Section B, vol. 271, pp. 96-101, 2012.

[144] Yu. I. Petrov and E. A. Shafranovsky, "On discontinuity of hyperfine fields at ${ }^{57} \mathrm{Fe}$ nuclei in bulk iron and aerosol $\mathrm{Fe}$ nanoparticles," Physics Letters A, vol. 336, no. 2-3, pp. 253258, 2005.

[145] Yu. I. Petrov, E. A. Shafranovsky, L. Casas, and E. Molins, "On the origin of discontinuity of the hyperfine fields at ${ }^{57} \mathrm{Fe}$ nuclei in bulk iron and aerosol Fe nanoparticles," Physics Letters A, vol. 375, no. 11, pp. 1421-1425, 2011.

[146] Yu. I. Petrov, E. A. Shafranovsky, Y. F. Krupyanskii, and S. V. Esin, "Discrete structure of the hyperfine field distribution at Fe nuclei in the bulk FePd alloy and its nanoparticles," Doklady Physical Chemistry, vol. 399, no. 1-3, pp. 269-274, 2004.

[147] Yu. I. Petrov and E. A. Shafranovsky, "Discrete distribution of hyperfine fields at the Fe nuclei in Fe-Co nanoparticles," Doklady Physical Chemistry, vol. 411, no. 2, pp. 339-344, 2006.

[148] J. Hesse and A. Rübartsch, "Model independent evaluation of overlapped Mössbauer spectra," Journal of Physics E, vol. 7, no. 7, pp. 526-532, 1974.

[149] R. A. Brand and G. Le Caër, "Improving the validity of Mössbauer hyperfine parameter distributions: the maximum entropy formalism and its applications," Nuclear Instruments and Methods in Physics Research Section B, vol. 34, no. 2, pp. 272-284, 1988.

[150] E. A. Shafranovsky and Yu. I. Petrov, "Change in a Hyperfine Field at the ${ }^{57} \mathrm{Fe}$ Nuclei in aerosol Fe nanoparticles when adding a small chromium admixture," Doklady Physics, vol. 54, pp. 111-114, 2009.

[151] K. P. Belov, Erscheinungen in Ferromagnetischen Metallen, Technik, Berlin, Germany, 1953.

[152] J. S. Smart, Effective Field Theories of Magnetism, W. B. Saunders, Philadelphia, Pa, USA, 1966.

[153] P. P. Craig, R. C. Perisho, R. Segnan, and W. A. Steyert, "Temperature and field dependence of hyperfine fields and magnetization in a dilute random substitutional ferromagnetic alloy: $\mathrm{Fe}_{2.65} \mathrm{Pd}_{97.35}$," Physical Review, vol. 138, no. 5, pp. A1460-A1471, 1965.

[154] G. K. Wertheim, Mössbauer Effect. Principles and Applications, Academic Press, New York, NY, USA, 1964. 
[155] R. E. Watson and A. J. Freeman, "Origin of effective fields in magnetic materials," Physical Review, vol. 123, no. 6, pp. 2027-2047, 1961. 


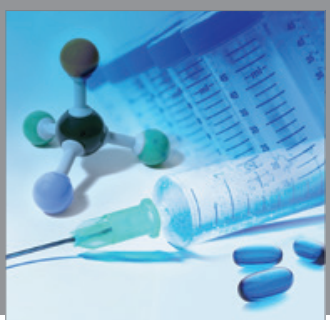

International Journal of

Medicinal Chemistry

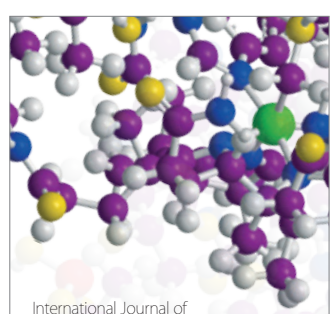

Carbohydrate Chemistry

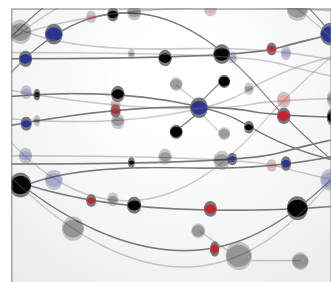

The Scientific World Journal
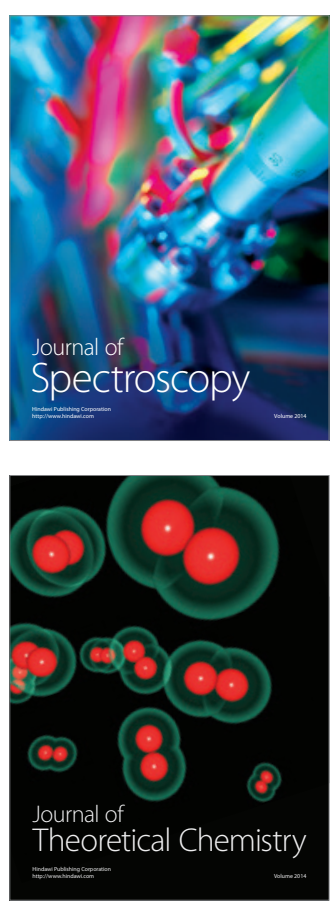
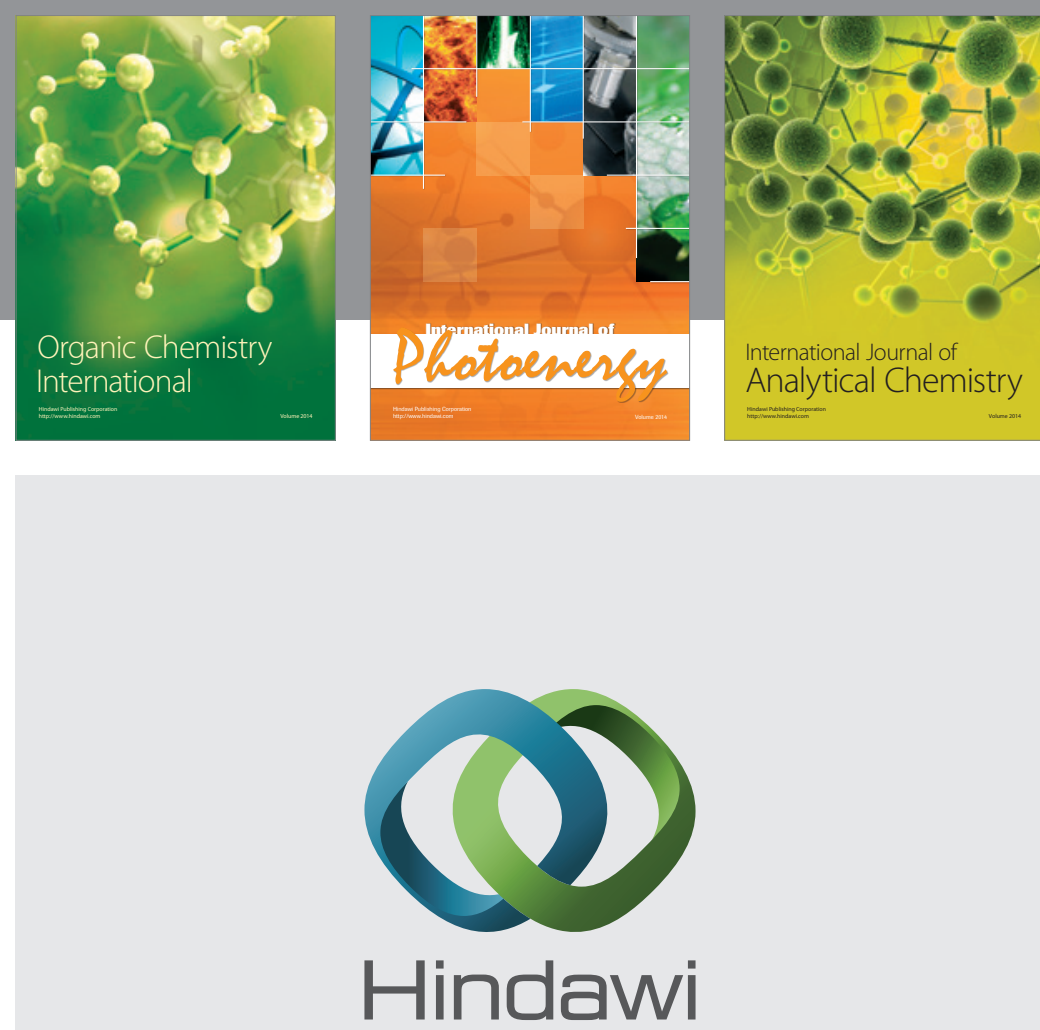

Submit your manuscripts at

http://www.hindawi.com
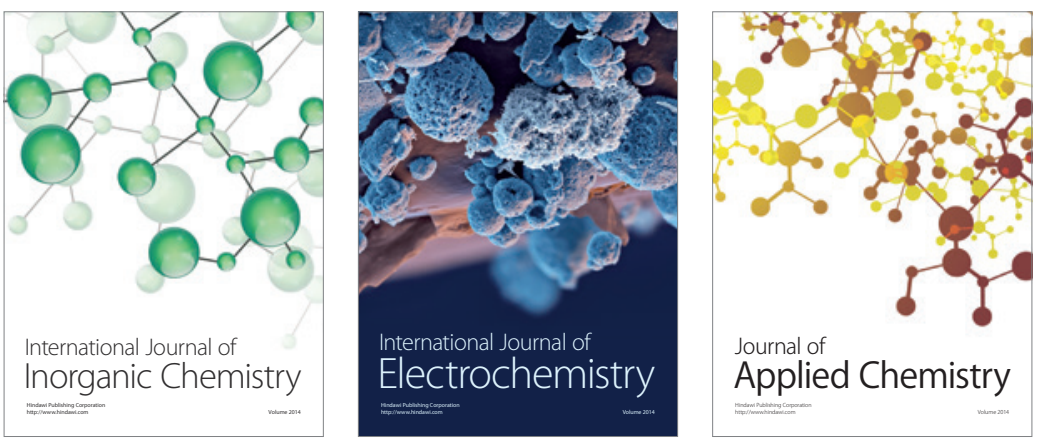

Journal of

Applied Chemistry
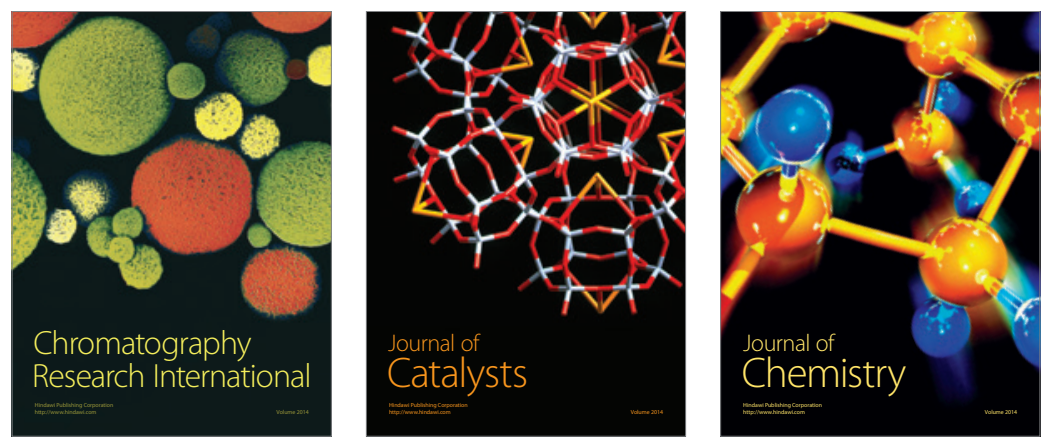
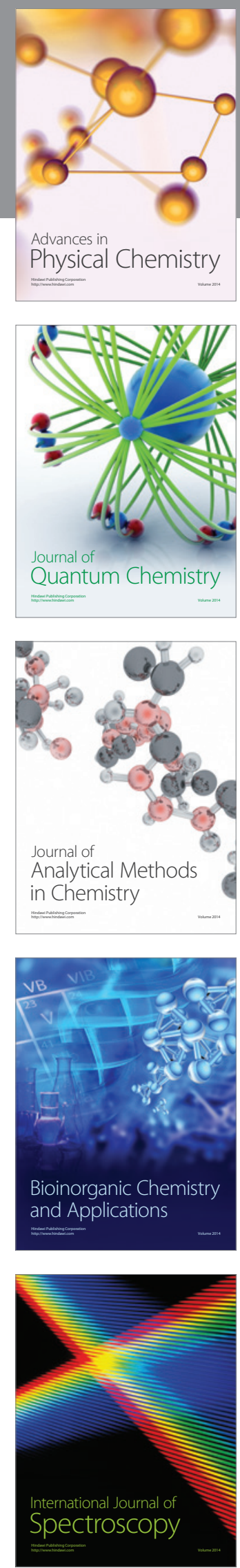